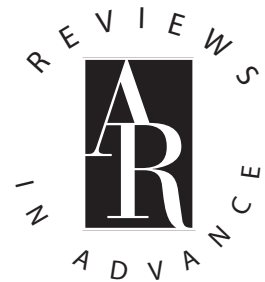

Annu. Rev. Mater. Res. 2014. 44:17.1-17.51

The Annual Review of Materials Research is online at matsci.annualreviews.org

This article's doi:

10.1146/annurev-matsci-122013-025024

Copyright (c) 2014 by Annual Reviews. All rights reserved online and in print.)
Review in Advance first posted online on May 7, 2014. (Changes may

still occur before final publication

\section{Stochastic Virtual Tests for High-'Temperature Ceramic Matrix Composites}

\author{
Brian N. Cox, ${ }^{1}$ Hrishikesh A. Bale, ${ }^{2}$ Matthew Begley, ${ }^{3}$ \\ Matthew Blacklock, ${ }^{4}$ Bao-Chan Do, ${ }^{5}$ Tony Fast, ${ }^{6}$ \\ Mehdi Naderi, ${ }^{5}$ Mark Novak, ${ }^{7}$ Varun P. Rajan, ${ }^{3}$ \\ Renaud G. Rinaldi, ${ }^{8}$ Robert O. Ritchie, ${ }^{2}$ \\ Michael N. Rossol, ${ }^{3}$ John H. Shaw, ${ }^{3}$ Oliver Sudre, ${ }^{1}$ \\ Qingda Yang, ${ }^{5}$ Frank W. Zok, ${ }^{3}$ and David B. Marshall ${ }^{1}$ \\ ${ }^{1}$ Teledyne Scientific Co. LLC, Thousand Oaks, California 91360; \\ email: brian.cox@teledyne.com \\ ${ }^{2}$ Department of Materials Science and Engineering, University of California, Berkeley, \\ California 94720 \\ ${ }^{3}$ Materials Department, University of California, Santa Barbara, California 93106-5050 \\ ${ }^{4}$ Sir Lawrence Wackett Aerospace Research Centre, School of Aerospace, Mechanical \& \\ Manufacturing Engineering, RMIT University, Melbourne, Victoria, 3001, Australia \\ ${ }^{5}$ Department of Mechanical and Aerospace Engineering, University of Miami, Coral Gables, \\ Florida 33124 \\ ${ }^{6}$ Woodruff School of Mechanical Engineering, Georgia Institute of Technology, Atlanta, \\ Georgia 30332-0405 \\ ${ }^{7}$ ATI Wah Chang, Albany, Oregon 97321 \\ ${ }^{8}$ MATEIS CNRS UMR5510, INSA-Lyon, F-69621 Villeurbanne, France
}

\section{Keywords}

stochastic properties, stochastic microstructure

\begin{abstract}
We review the development of virtual tests for high-temperature ceramic matrix composites with textile reinforcement. Success hinges on understanding the relationship between the microstructure of continuous-fiber composites, including its stochastic variability, and the evolution of damage events leading to failure. The virtual tests combine advanced experiments and theories to address physical, mathematical, and engineering aspects of material definition and failure prediction. Key new experiments include surface image correlation methods and synchrotron-based, micrometer-resolution $3 \mathrm{D}$ imaging, both executed at temperatures exceeding $1,500^{\circ} \mathrm{C}$. Computational methods include new probabilistic algorithms for generating stochastic virtual specimens, as well as a new augmented finite element method that deals efficiently with arbitrary systems of crack initiation, bifurcation, and coalescence in heterogeneous materials. Conceptual advances include the use of topology to characterize stochastic microstructures. We discuss the challenge of predicting the probability of an extreme failure event in a computationally tractable manner while retaining the necessary physical detail.
\end{abstract}




\section{INTRODUCTION}

\subsection{The Purpose, Nature, and Challenges of Virtual Tests}

One role of a virtual test, as its name suggests, is to replace a real engineering test with a computer simulation. Ideally, the simulation would predict engineering properties ab initio with sufficient fidelity to make the real test unnecessary. More realistically, a virtual test calibrated with a few real tests reduces, perhaps by an order of magnitude or more, the matrix of real tests needed to ensure safe use of the material (1).

Of equal interest is the possibility that a virtual test can function as a tool for optimizing material design (2). Indeed, a virtual test can yield much richer information about the correlation between the microstructure of a material and its performance than experiments can: In the virtual test, we have full knowledge of the microstructure and its effect on the details of failure mechanisms, whereas in the real test, such effects are often concealed in the interior of the specimen.

To achieve the required level of fidelity, a virtual test must replicate the microstructure and the evolution of discrete damage events in detail. In particular, to predict the variability of engineering tests, which determines safety margins, the virtual test must account for stochastic variations in the material's microstructure, which are an important source of performance variability.

In a common paradigm based on Monte Carlo methods, the virtual test is built by the following steps (e.g., References 1, 3-7).

1. The microstructure of the real material is measured and characterized in all the details that are likely to influence predicted material behavior.

2. The measured microstructural characteristics, as they are stochastic, are fitted by distribution functions.

3. An ensemble of virtual specimens is generated by the Monte Carlo method. Each specimen, although distinct from any real specimen, nevertheless possesses a microstructure with the same statistics as those measured on the real specimens.

4. The material properties associated with the virtual specimen, including, for example, the fracture laws that govern crack initiation and propagation, are calibrated by real tests.

5. Damage evolution is simulated for each virtual specimen in the ensemble, taking explicit account of the influence of the particular local microstructure present in any region of the specimen on the damage events that are occurring in that region.

6. The virtual test is validated by comparing the statistics of failure predicted for the ensemble of virtual specimens against those from tests on real specimens.

A number of challenges confront those constructing virtual tests. First, to study the effect of microstructure on damage, the microstructure must be measured with resolution equal to the dimensions of heterogeneities, e.g., 1-100 $\mu \mathrm{m}$ in the case of typical metal alloy grains or $1-10 \mu \mathrm{m}$ in the case of continuous fibers in a composite. One of the most dramatic advances in the field of virtual tests is that, over the past 5 years, nondestructive $3 \mathrm{D}$ measurements of microstructure with a resolution approaching $1 \mu \mathrm{m}$ have become feasible by using high-energy radiation sources (8-16). Damage events and the local microstructure with which they interact are being revealed nondestructively within the interiors of specimens, with a resolution better than $1 \mu \mathrm{m}$, a level of detail in information that is having a transformative effect on the viability of virtual tests.

Second, realistic simulations of complex damage events require advanced and well-chosen computational methods. In the heterogeneity of an advanced composite, failure occurs by a complex combination of interacting cracks and local damage events, which are governed by nonlinear material behavior. Key advances in theory have enabled a realistic depiction of the nonlinear mechanics

Cox et al. 
of crack initiation, growth, bifurcation, and coalescence; critically, the location and path of each crack are determined by local stress conditions during a simulation, rather than being prescribed in advance (17-26). Fidelity in simulations was impossible before such generality was achieved.

Third, the value of virtual tests depends strongly on the speed at which they can be executed. In the Monte Carlo approach outlined in steps 1-6 above, the influence of random microstructure is computed by analyzing the statistics of a large number of simulations, each of which tracks damage evolution in real space in a randomly generated sample. To establish safety margins in cases in which high degrees of safety are required, e.g., in the case of vehicles for human transportation, the virtual test must predict the probability of rare events. If the probability of an extreme case is $10^{-6}$, for example, then predicting failure by Monte Carlo methods requires $>10^{7}$ simulations to assure that the extreme case is sampled a statistically significant number of times. In applications to material optimization, a search over different combinations of material selection and fiber architecture, for example, leads to the need for solving similarly large computational ensembles. If each case in the Monte Carlo ensemble requires $1 \mathrm{~h}$ of computation time, progress is not feasible; a good near-term target for computational times is $1 \mathrm{~s}$.

Fourth, failure mechanisms in all engineering materials involve a complex hierarchy of material features and damage events at different spatial scales (27-30). The term microstructure as it is used in this article covers phenomena at scales of $\sim 0.1-1 \mathrm{~mm}$ (e.g., a fiber ply or fiber tow), 1-10 $\mathrm{m}$ (e.g., a single fiber), or $\sim 1-1,000 \mathrm{~nm}$ (e.g., a fiber/matrix interface or fiber coating). Pragmatic choices are necessary: Not all levels of complexity can be supported in a virtual test, especially if the virtual test must treat large ensembles of virtual specimens. Thus, step 1 of the paradigm presented above requires identification of the details that will be influential for predicting a particular property $(1,6)$; just which details are influential and which can be omitted in the interest of achieving a tractable model will be the subject of research for a long time to come.

\subsection{The Nature and Representation of Defects}

Every defect in a material is a deviation in the geometrical arrangement of its constituents at some scale. For example, at the scale of fiber bundles or plies in a composite, defects include local regions of fiber waviness or misalignment; at the scale of the fiber bundle, defects include variance in the packing arrangement of fibers; and at the scale of the single fiber, defects include unusually large grains within the fiber (Figure 1). Defects in a composite include variance in the geometrical disposition of the matrix phase, e.g., voids, or in the configuration of different phases in a hybrid matrix. And defects also include the possibility of preexisting cracks at all scales (e.g., matrix cracks at the tow or ply scale or nanoscale cracks within fibers), which can again be considered as geometrical variances, in this case discontinuities in the material disposition. Key to progress in establishing virtual tests is the ability to measure all such defects (31-35), which trigger failure mechanisms and compromise strength and life (e.g., References 36-43).

The general approach to stochastic virtual tests reviewed in this article consists of determining defects as geometrical variances in the material and then predicting their effect on properties (i.e., their strength as defects) by high-fidelity simulations of failure. For pragmatic reasons, this method is embedded in a top-down strategy (45): The defects are sought first at the largest scale (at which geometrical variance is easiest to measure) and then at progressively finer scales. In due course, with perfect experiments, the strategy could in principle be pursued down through all scales to the atomic scale. However, in reality, at any time in the history of developing virtual tests, the geometry cannot be measured exhaustively at scales beneath some threshold. Yet evidence implies indisputably that defects at finer scales do exist, even if they are as yet invisible, and that they govern some aspects of failure.

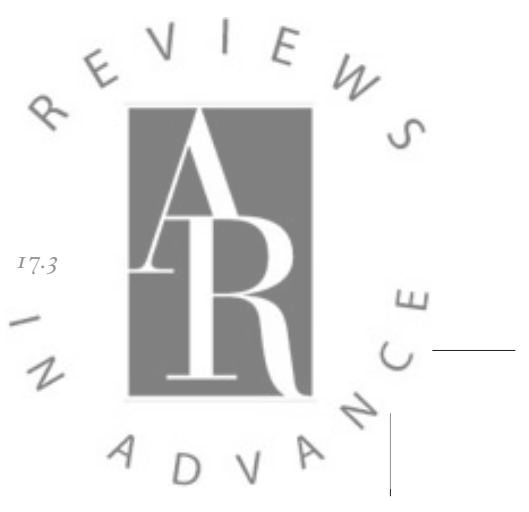



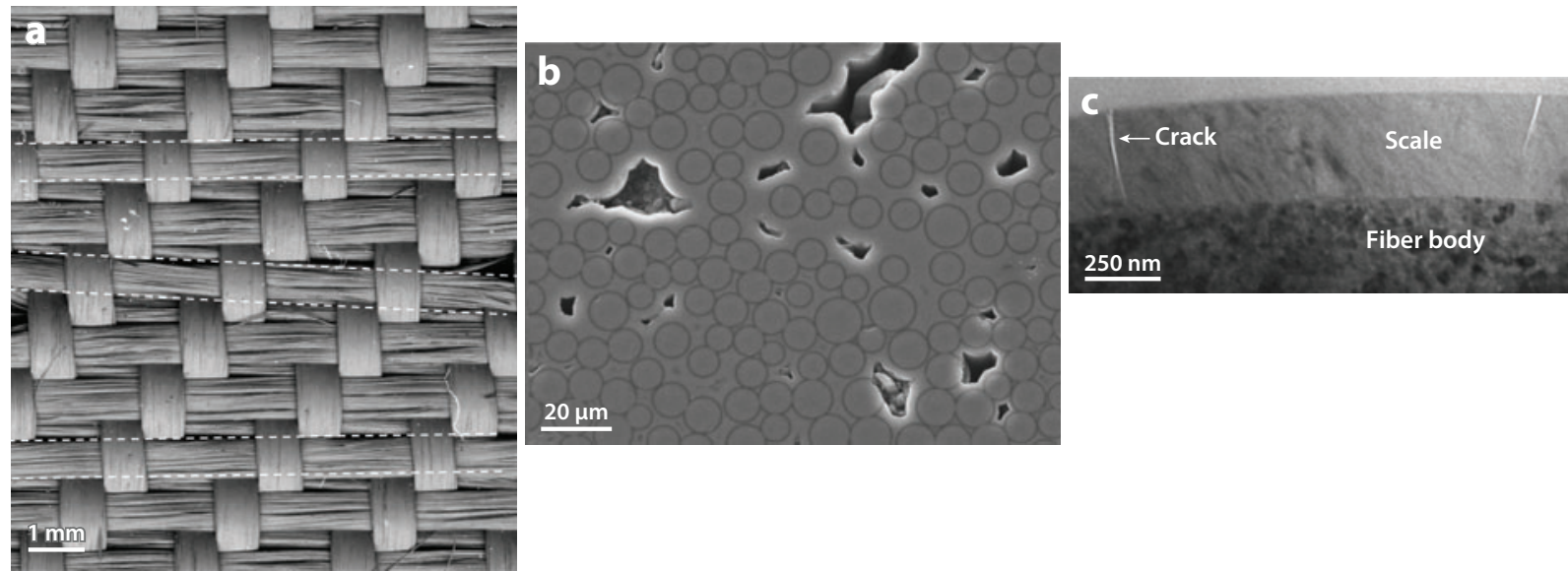

Figure 1

Examples of defects consisting of variations in geometry. (a) Irregularity in the alignment and shapes of fiber tows. The white dashed lines outline three weft tows that are nominally parallel. (b) Irregularity in the size and positioning of fibers within a ply or bundle, which has led to uneven matrix infiltration and residual porosity. (c) Cracks (on the nanometer to micrometer scale) in an oxide scale that formed on a $\mathrm{SiC}$ fiber during high-temperature oxidation. Image in panel $c$ courtesy of Dr. Randy Hay; see also Reference 44 for a similar image.

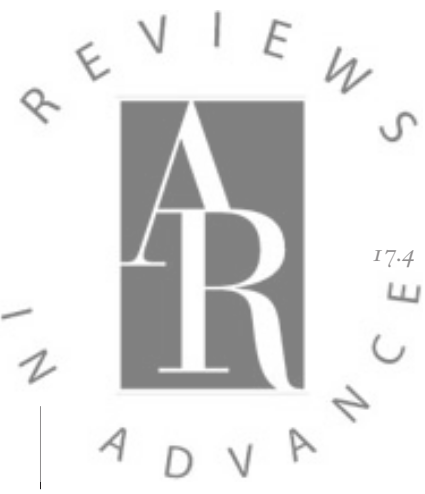

Thus, the stochastic virtual test combines explicit representations of defects that can be seen and measured with implicit representations of defects whose presence is known but that are too fine to characterize with current experiments. The latter class of defects is treated in the traditional manner: They are represented by knockdowns in material strength, measured by appropriate tests of the composite or of its constituent phases. In the present status of development, a prime example of implicit representation of defects is the use of distributions of fiber strength. Because the process of experimental measurement of defect-moderated strength distributions and the introduction of the strength distributions into simulations is well known $(46,47)$, this topic is treated only briefly. Instead, this article focuses on predicting how defects whose geometry can currently be determined experimentally affect composite properties, given mathematical representations of the defect geometry.

\subsection{Virtual Tests for High-Temperature Continuous-Fiber Composites}

Virtual tests are of special value for high-temperature materials, e.g., the current generation of integral textile ceramic matrix composites (48-50), with potential use temperatures ranging up to $1,500^{\circ} \mathrm{C}(51-53)$. Strong continuous-fiber bundles (scale of $0.1-1 \mathrm{~mm}$ ) are woven in customdesigned 3D patterns, with individual bundles $(a)$ oriented to follow the primary load paths in a component to maximize its strength and $(b)$ interlocked with one another to prevent catastrophic separation when damaged. Larger interstices between the fiber bundles may be partially filled with randomly oriented, fine reinforcing rods (scale of 1-10 $\mu \mathrm{m}$ ), inhibiting local cracking under thermal shock. Coatings applied to individual fibers (scale of $0.1-1 \mu \mathrm{m}$ ) inhibit chemical reactions and ensure that the interfaces between the fibers and the matrix remain weak, allowing ductile response through matrix cracking and frictional pullout of crack-bridging fibers. The remaining space between coated fibers, fiber bundles, and reinforcing rods is filled with a ceramic matrix material, which may be a hybrid containing, for example, graphitic sheets that inhibit oxygen ingress (scale of 1-100 nm) (54). Thus, like many natural materials, these new ceramic composites

Cox et al. 
achieve robustness through complexity: Their hierarchical, hybrid microstructure impedes the growth of local damage and prevents the large fatal cracks that are characteristic of brittle materials.

Whereas mechanisms of failure in composites that act at room temperature can be readily determined either by modern 3D imaging or by destructive sectioning following interruption of tests, mechanisms acting at high temperatures are much more difficult to probe. Even surface observations tend to be obscured by radiation if observed in situ, whereas observations at room temperature following test interruption are influenced to an unknown degree by the thermomechanical effects of cooling from the test temperature. The virtual test offers the possibility of probing details of damage mechanisms for different temperature and loading histories by using simulations coupled to relatively simple surface observations on real specimens. Nevertheless, advancing test methods applicable to high temperatures remains critical: The proven fidelity of a virtual test can never exceed the ability to identify the mechanisms that must be modeled by direct experimentation.

In the case of virtual tests for continuous-fiber composites, including composites reinforced by laminated fiber plies and textile preforms, the high resolution now available in micrometerresolution $\mathrm{X}$-ray computed tomography $(\mu \mathrm{CT})$ imaging systems is yielding details of the stochastic variability of plies and fiber tows and of even the spatial distribution of individual fibers. Investigators have measured characteristics of textile geometry and tow deformation $(8,13,55)$, as well as porosity $(56)$ and its changes during processing steps $(56,57)$. The same technique is now yielding $3 \mathrm{D}$ images of damage captured in situ under load at very high temperatures, which is key to informing simulations of damage evolution (58).

These and other studies have also addressed achieving feature definition in ceramic composites (10), which is often made difficult by low X-ray absorption contrast between the constituent materials. In recent work, fiber tows were made to stand out by imaging composites with partially formed matrices (9).

The importance of geometrical defects in fibrous composites is already well understood from decades of research on polymer and ceramic composites: Variability in fiber positioning is a principal source of scatter in composite performance. In polymer composites, for example, both strength in monotonic compression and fatigue life in compression-compression fatigue are influenced by local misalignments in fiber bundles, which lead to kink band instabilities (36, 59-62). Kink bands have not been observed in ceramic composites, but tow straightening occurs under tension, implying local damage within tows that is driven by local shear stresses and that correlates with the degree of tow misalignment. In both polymer and brittle matrix composites, fracture of a fiber ply or tow loaded in tension perpendicular to the fiber direction tends to initiate at and propagate through locations where the fiber spacing is unusually small (63). The importance of avoiding fiber misalignments and wrinkles and other fiber deployment defects has become well recognized by those involved in modeling and controlling the textile-forming process (64).

In this article, we review the development of a virtual-test system for high-temperature, textilebased ceramic matrix composites, along with the advances in theory and experiment that make such a goal reachable. We describe the stochastic character of fiber reinforcement, new experimental methods by which information-rich data can be acquired at high temperatures, efficient computational methods for simulating the development of complex, discrete crack systems, and issues related to predicting extreme behavior.

\subsection{Complex Issues Require Coordinated Research Structures}

By necessity, developing a virtual-test system requires the cooperation of researchers from different disciplines and institutions. Organizing that cooperation to assure the achievement of an

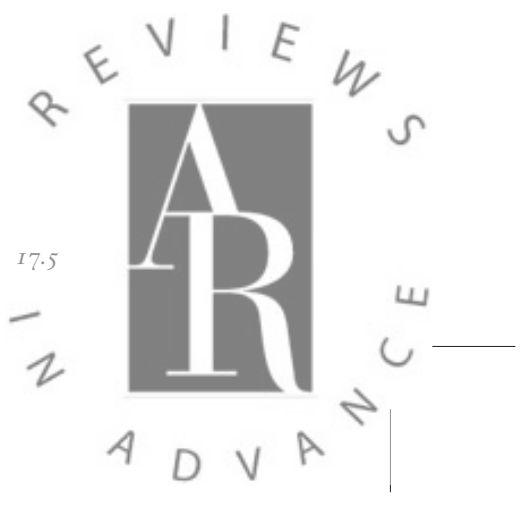




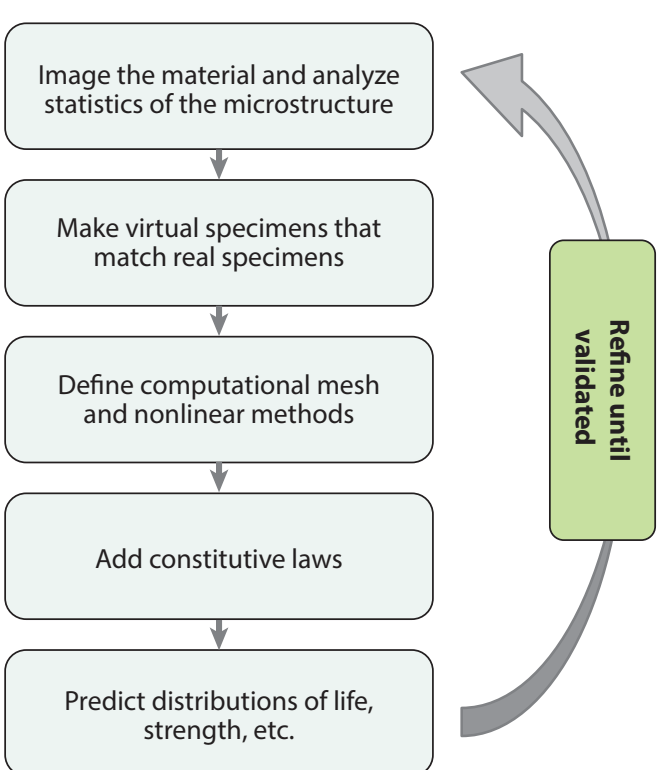

Figure 2

A pipeline approach to assembling a cross-disciplinary virtual-test system.

overarching goal and the production of a virtual-test system that can be sustained and built upon in the future is a major challenge. An idea pursued in one project was to seek effective cooperation of different researchers by organizing research along a pipeline model, identifying the contributions from disciplines as sequential steps in an overall process (Figure 2). The sections of this article in fact follow the logic path of Figure 2, but with some deviations, because experimental and theoretical activities that are emphasized in single elements of the pipeline also tend to have at least a minor role in other elements; the structure of the problem is not entirely sequential. Section 2 describes the characterization of stochastic textile microstructures. Section 3 considers the problem of using such data to construct virtual specimens. Section 4 relates new experiments that probe the details of failure at high temperature and thereby guide the formulation of computational methods for analyzing the virtual specimens. Section 5 treats the challenge of calibrating constitutive laws for the constituent materials in virtual specimens, thus completing their definition. Section 6 describes new computational techniques for solving systems of interacting microcracks and thus executing tests of virtual specimens. Section 7 summarizes the challenge of validating the fidelity of a virtual test. Section 8 discusses the use of virtual tests of stochastic virtual specimens for predicting distributions of engineering properties. The virtual-test system is described primarily as required to address events at the scale of a fiber tow (with a typical cross section of $0.1 \times 1 \mathrm{~mm}^{2}$ ). A final section (Section 9) summarizes the distinct challenges of progressing down to the scale of a single fiber $(1-10 \mu \mathrm{m})$.

Throughout this article, we emphasize the necessity of extending engineering practice beyond the current paradigm of combining idealizations of composite structure, analysis by the finite-element method (FEM), and conventional engineering tests. Such traditional approaches (e.g., the textile composite analysis in References 65 and 66) achieve only a limited account of the relationship between microstructure and material performance. More realistic virtual tests demand new experiments that can reveal the coupling between $(a)$ mechanisms of failure and the microstructural features that govern them at relevant spatial scales and (b) new concepts and

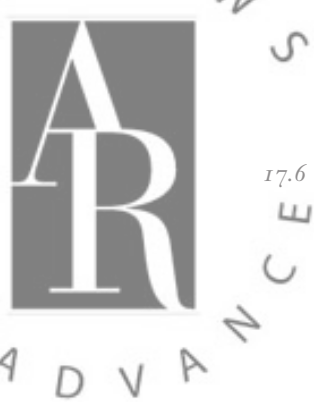

Cox et al. 
analytical models for describing probabilistic aspects of the material and failure events. The most important new experiments and theory tend to have been developed in other disciplines, with little prior use in engineering.

\section{THE STOCHASTIC CHARACTERISTICS OF TEXTILE COMPOSITES}

Variability in the spatial deployment of fibers (geometrical defects in the textile) contributes substantially to variance in the properties of their composites. In the virtual-test pipeline, the stochastic characteristics of the fiber reinforcement are determined experimentally and summarized in a standard, simple data set, which becomes the input for the next step, the process of setting up virtual specimens that possess stochastic microstructure with the same statistics.

Determination of stochastic microstructural characteristics is a very active field, although the majority of this effort has been focused on polycrystalline materials, especially metal alloys (6769). Continuous-fiber composites present challenges fundamentally different from those posed by alloys, both in the type of experiment that most successfully characterizes microstructural variance and in the concepts used to deduce statistics from raw data that are germane to material performance.

Although acquisition of 3D data on alloy microstructure has been based on serial sectioning $(70,71)$, this method has limitations in application to fiber composites: If fibers change positions between successive cross sections, the correct association of a fiber on one cross section with the same fiber on another cross section may be difficult to establish, especially if fibers are discontinuous or broken. If fibers happen to lie almost parallel to a cross section, the positions of their centers of mass can be difficult to determine even on a single cross section, and following individual fibers through successive cross sections is impossible.

Thus, the availability of $\mu \mathrm{CT}$ offers a revolution in data acquisition for fibrous composites. In a high-intensity synchrotron beamline, data can be acquired at submicrometer resolution with such speed that scanning many cubic millimeters of material is routine; such a scan size supports detailed analysis of the statistics of fiber randomness $(9,12)$. The $\mu$ CT data can quantify stochastic fiber microstructure at the tow scale and at the scale of an individual fiber (Section 9). Optical images of external surfaces of a fabric, when interpreted in conjunction with $\mu \mathrm{CT}$ data, can extend the quantification to the subcomponent scale, i.e., hundreds or thousands of unit cells.

However, even with these modern data sources, continuous-fiber composites pose challenges not seen in polycrystalline materials. The continuity of fibers tends to dampen positional deviations along the fiber direction, with the result that deviations occur over characteristic wavelengths that can be very long relative to the dimensions of scanned volumes. In contrast, spatial correlations between different fiber tows or single fibers within a plane that lies perpendicular to their axes (a normal section) are characterized by wavelengths that are short compared with the dimensions of scanned volumes. Thus, in the experiments reviewed in this section and in Section 9, whether at the scale of subcomponents, at the scale of the unit cell, or at the scale of the fiber, rich information can be obtained about geometrical variance occurring on normal sections, whereas information about variations along the fiber direction is quite limited. The extreme anisotropy in data density is as severe as the anisotropy that can arise in mechanical properties and is ultimately attributable to the same fact: The fibers are continuously connected in only one direction. The statistics selected to characterize geometrical variance are concomitantly anisotropic: Many degrees of freedom can be meaningfully employed for normal sections, whereas one or two scalar parameters describe variations along the fiber direction.

In nominally periodic textiles, the positions and shapes of tows can be expressed as the sum of (a) nonstochastic, periodic variations associated with the nominal periodicity of the textile

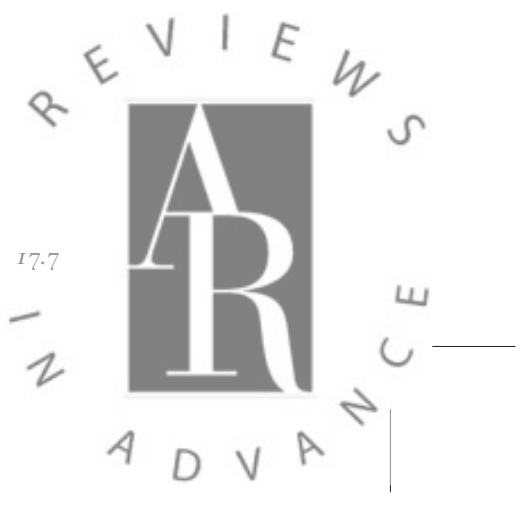


architecture and $(b)$ stochastic deviations from these average trends, which vary randomly through the fabric. A convenient and intuitively appealing treatment of the deviation divides it in turn into a superposition of short-range and long-range deviations. The short-range deviations, determined by $\mu \mathrm{CT}$, are those arising within a unit cell and include variations in the shapes as well as the positions of tows. The long-range deviations, determined from optical surface images, are the displacements of tows over wavelengths that are greater than the dimensions of the unit cell.

\subsection{Characteristics at the Scale of the Unit Cell}

Geometrical details of the fiber reinforcement in textile composite specimens somewhat larger than but comparable in size to a single unit cell (several millimeters in length) can be determined most satisfactorily by using $\mu \mathrm{CT}$, which reveals comprehensive shape and positional information on the fiber tow scale. Information concerning matrix voids, individual fibers, and fiber coatings also emerges for even smaller scales, down to $1 \mu \mathrm{m}(10,11,72)$. Figure $3 \boldsymbol{a}$ shows typical 2D image slices of a $3 \mathrm{D}$ woven carbon/SiC composite, and Figure $3 \boldsymbol{b}$ shows a $3 \mathrm{D}$ image of a unit cell. This material was fabricated with only enough matrix to rigidify the structure, which simplified the identification of tow domains (9).

For the shapes typical of tow cross sections in textiles, statistical analysis of the shape and positioning of the fiber tows can be based on analysis of sequences of cross sections along the tow

a

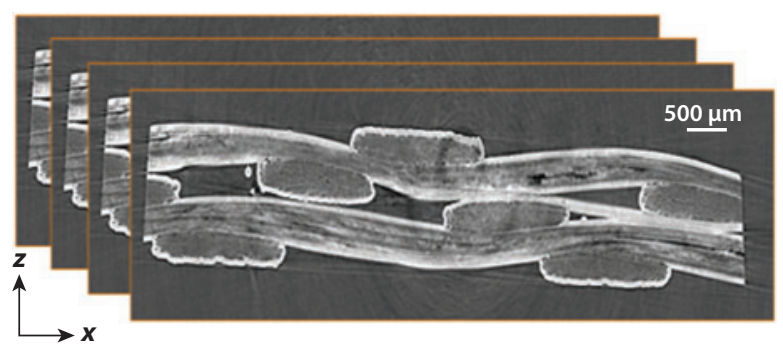

C

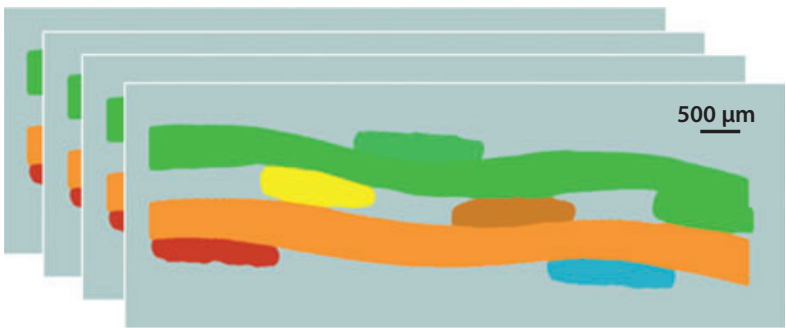

b

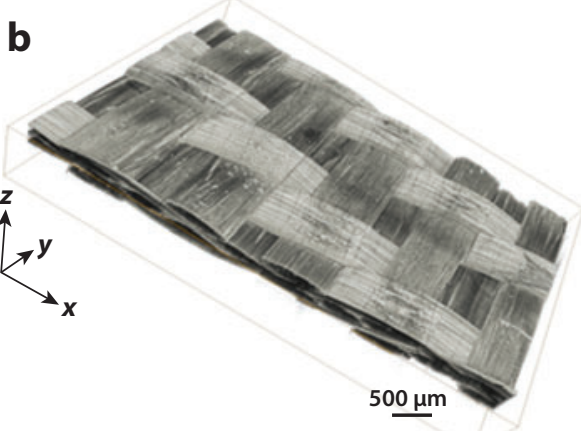

d

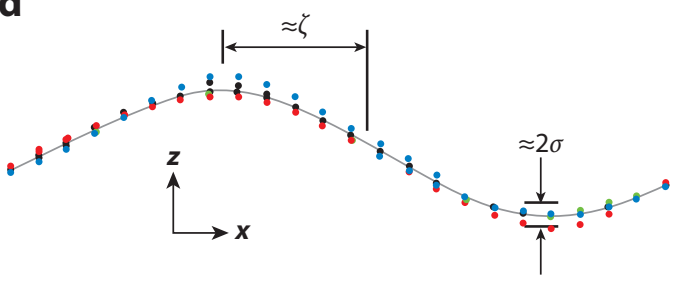

Figure 3

Analysis of $\mu \mathrm{CT}$ data for a carbon/SiC woven composite consisting of fiber tows coated by a thin layer of matrix material. (a) Image slices. (b) The reconstructed 3D image. (c) Segmentation of the image into tow domains. (d) Inferred statistics for the $z$-coordinate of the warp tow locus: The curve shows the average trend for warp tow over one reference period (which spans one unit cell of the textile architectuke), whereas blue, red, green, and black dots show data for different warp tow segments. The deviations of data for each tow from the mean fisend have root-mean-square deviation $\sigma$, whereas the distance along the tow over which deviations are correlated defines the correlationlength $\zeta$ (e.g., deviations of blue data vary over length $\zeta$ ).

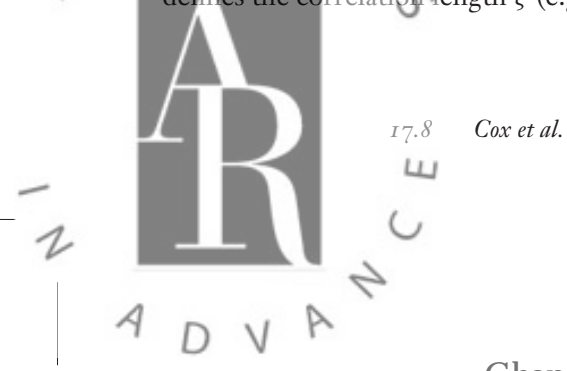


axis. For the convex or mildly reentrant cross-sectional shapes typical of tows, moment expansions of the shape function $\Omega(y, z)$ are a good general description:

$$
\begin{gathered}
\omega_{p q}=\iint y^{p} z^{q} \Omega(y, z) d y d z \\
\Omega(y, z)= \begin{cases}1 & \text { if }(y, z) \text { lies inside the tow, } \\
0 & \text { if }(y, z) \text { lies outside the tow, }\end{cases}
\end{gathered}
$$

where the nominal tow direction is the $x$-axis and $p$ and $q$ are integers (the moment orders). In cases in which tow cross sections are free of reentrant features, a serviceable truncation of the moment expansion can be obtained by fitting ellipses to data (even though the cross sections may deviate from elliptical). A fitted ellipse defines five of the six moments of order $o=p+q \leq 2$, with the sixth moment having a fixed relation to the other five, because of the particular shape of the ellipse. Ellipse fitting yields the data set

$$
\left\{y^{(m)}, z^{(m)}, A^{(m)}, a r^{(m)}, \theta^{(m)}\right\},
$$

where $y^{(m)}$ and $z^{(m)}$ are the center-of-mass coordinates and $A^{(m)}, a r^{(m)}$, and $\theta^{(m)}$ are the area, aspect ratio, and orientation, respectively, of section $m$, one of a sequence of cross sections on a discrete grid. In cases in which cross sections possess highly distorted shapes, such as strongly reentrant features, characterization should be based on higher-order moment expansions or on other generalized shape descriptors (73).

For a textile that is nominally periodic, each variable in Equation 2 can be decomposed into nonstochastic, periodic variations and nonperiodic, stochastic variations (Figure 3d ) (9):

$$
\begin{gathered}
(y, z)^{(m)}=\left\langle(y, z)^{(m)}\right\rangle+(\delta y, \delta z)^{(m)}, \\
A^{(m)}=\left\langle A^{(m)}\right\rangle+\delta A^{(m)}, \\
a r^{(m)}=\left\langle a r^{(m)}\right\rangle+\delta a r^{(m)}, \\
\theta^{(m)}=\left\langle\theta^{(m)}\right\rangle+\delta \theta^{(m)},
\end{gathered}
$$

where $\langle\ldots\rangle$ indicates an average value. The periodic trends are compiled by exploiting the nominal translational invariance of the textile: Data from all points on nominally equivalent tows throughout the specimen are collated onto a single periodic cycle of that type of tow, a process termed reference period collation $(9,74)$. This process maximizes the information content of the relatively small specimens that can be imaged at high resolution. The stochastic deviations from the periodic trends consist of the set of standard deviations, $\sigma_{y}^{(k)}$, etc., of the cross-sectional characteristics (Figure 3d). For the materials of Figure 3, the deviations are independent of location $x$ along the tow. The superscript $k$ denotes a tow genus, i.e., a set of warp or weft tows that are nominally equivalent to one another in the periodic pattern of the weave. Another statistic of interest is the correlation length $\zeta_{y}^{(k)}$ for each cross-sectional characteristic; $\zeta_{y}^{(k)}$ measures the distance along the tow over which correlations between the values of deviations at two points become weak (Figure 3d).

After analysis has determined the important statistical variables for a given data set, the stochastic character of the material can be summarized by a specific, quite small set of statistical variables.

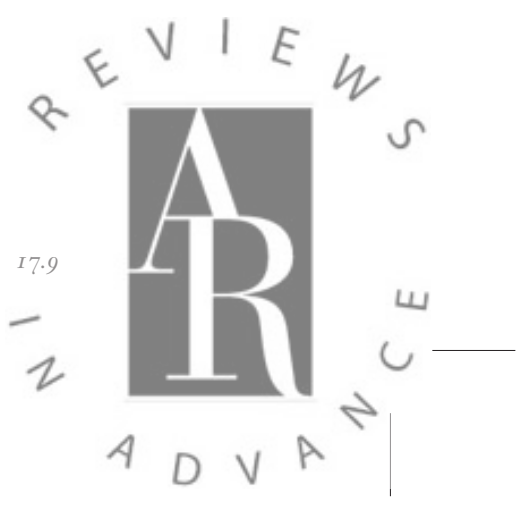


For the materials of Figure 3, the representational set of experimental statistics is

$$
\begin{aligned}
& D=\left\{D_{k}, k=1, \ldots, N_{g}\right\}, \\
& D_{k}=D_{k}^{(a v)} \cup\left\{\sigma_{\xi}^{(k)}, \sigma_{z}^{(k)}, \sigma_{A}^{(k)}, \sigma_{a r}^{(k)}, \sigma_{\theta}^{(k)}, \zeta_{\xi}^{(k)}, \zeta_{z}^{(k)}, \zeta_{A}^{(k)}, \zeta_{a r}^{(k)}, \zeta_{\theta}^{(k)}\right\}, \\
& D_{k}^{(a v)}=\left\{\left\langle\xi^{(k)}\right\rangle_{i},\left\langle z^{(k)}\right\rangle_{i},\left\langle A^{(k)}\right\rangle_{i},\left\langle a r^{(k)}\right\rangle_{i},\left\langle\theta^{(k)}\right\rangle_{i}, \quad i=1, \ldots, N_{\varphi}\right\} .
\end{aligned}
$$

Here, $D_{k}$ contains the data for tow genus $k$, with number $N_{\mathrm{g}}$; the superscripted (av) indicates the contribution of an averaged quantity; $\xi \equiv y$ or $x$, depending on the nominal direction of tows of genus $k$; the index $i$ denotes one of $N_{\phi}$ sections spaced equally along a single reference period of a tow; $\sigma$ denotes the root-mean-square deviation (RMSD) of a variable (e.g., $\sigma_{\xi}$ and $\sigma_{z}$ refer to the deviations $\delta \xi$ and $\delta z$, respectively); $\zeta$ denotes the correlation length for the deviations of a variable along a tow (e.g., $\zeta_{\xi}$ and $\zeta_{z}$ refer to the deviations $\delta \xi$ and $\delta z$, respectively); and the quantities in parentheses $\langle\ldots\rangle$ refer to the averaged quantities of Equation 3, which express nonstochastic periodic variations as functions of position $i$ along the reference period of a tow (Figure $3 \boldsymbol{d}$ ).

The data set of Equation 4 provides a complete summary of stochastic tow positioning and shape in a relatively small number of parameters, comprising the deterministic variations of each tow over a single period (defined on a grid of typically 20 points) and ten other scalar parameters that contain all information about stochastic variations at the unit cell scale. This compact data set defines the complete 3D shapes of tows and the statistics of their shape deviations. It provides a standard protocol for passing information from experiment to a virtual-specimen generator and thence to analyses of how stochastic microstructure at the unit cell scale affects performance.

Establishing a formal standard set of such statistics facilitates the comparison of different materials and allows statistics to be archived in a consistent format. Equation 4 is proposed as a candidate standard set for the tow-scale geometry of periodic textiles. However, one must also recognize the possibility that further research on how randomness in microstructure affects the properties of composites will necessitate augmentation of the standard set to include other metrics.

\subsection{Large-Scale Stochastic Weave Characteristics}

Although the high resolution of $\mu \mathrm{CT}$ is needed to resolve tows and fibers, $\mu \mathrm{CT}$ is not a practicable approach for gathering data on the large-scale deformations seen in specimens containing hundreds or thousands of unit cells or more; the scan times are prohibitive. A useful alternative can be based on digital image analysis of optical images, which combines optical resolution with large field of view. For thin textile preforms (e.g., Figure 3), surface imaging accesses a sufficient fraction of all tows to characterize much of the overall fabric deformation.

Surface images captured in stereo pairs by viewing the surface at two angles can be analyzed to determine both in-plane and out-of-plane variations in a sample. Isolating the out-of-plane variations or topography reveals features that correlate with the textile architecture (Figure $4 a$ ). The features can be automatically identified as tows of a specific genus by analyzing their spatial relationship to the nearest other features (a graph or neighbor-mapping analysis). The colored patches in Figure $4 \boldsymbol{a}$ show the outlines of tow crowns, which are thresholded extremities of surface height. Color-coding separates the crowns into warp and weft genuses.

From these base data, long-range variations in the topography of the surface can be determined by averaging the heights of nominally equivalent features over a suitable gauge length. Examples appear in Reference 75.

In-plane deviations are evaluated as departures from a hypothetical perfectly periodic textile, which is generated by first computing the average separations between rows of features, which define lattice parameters, and then determining a global Cartesian coordinate system, which is

Cox et al. 
a

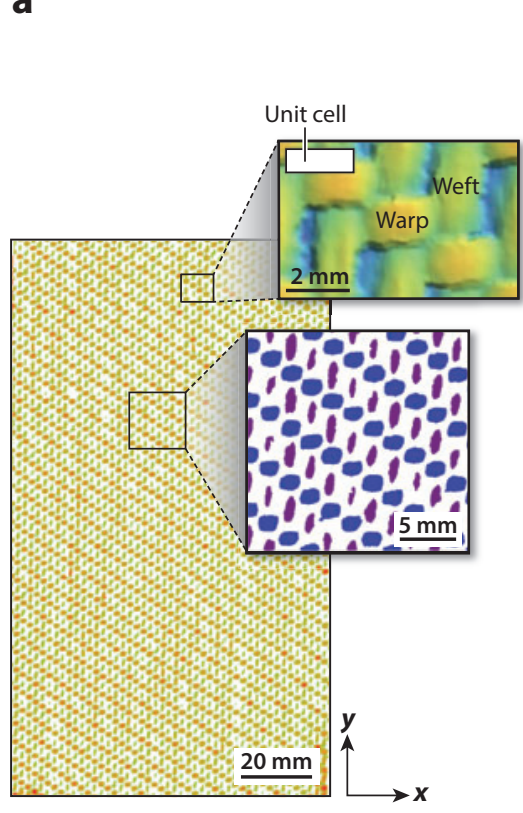

b

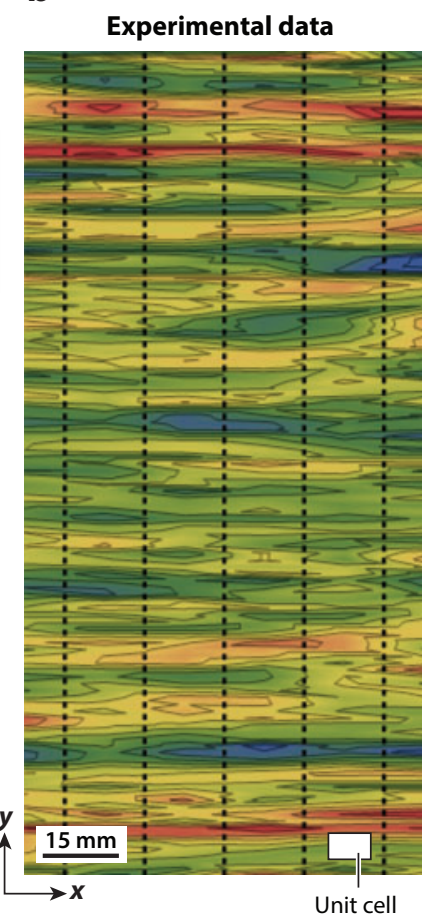

C Reconstruction (one instantiation)

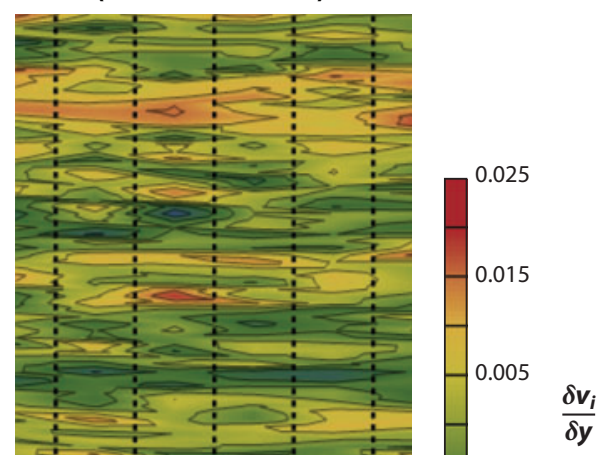

Figure 4

Digital image analysis of a panel of carbon/SiC angle interlock material similar to that represented in Figure 3 (with smaller weft tow spacing but otherwise identical architecture). The panel contains approximately 120 weft tows and 120 warp tows, which occupy approximately 10 unit cells in the warp direction and 30 unit cells in the weft direction. (a) Identification of the crowns of tows of different genuses, whose centers of mass yield a discrete sampling of positions for tows. The main image shows the pattern of features over the whole sample. Insets show enlargements of the pattern of thresholded features (the different color set displayed is not significant) and of the textile surface topography. (b) Spatial derivatives of in-plane deviations of tow positions, determined relative to a hypothetical perfectly periodic structure, reveal variations in the tow packing density. The dashed lines indicate scan lines along which variations are decomposed by Fourier analysis. (c) Tow packing variations in a virtual specimen, which is a different instantiation of the stochastic variations found in panel $b$ but is constructed from the same statistical distributions (see Section 3).

defined to be the system from which rows of features show the least angular deviation. With the perfect lattice thus defined, the deviation of the center of mass of each feature from the location expected for that feature on the perfect lattice is computed. The in-plane deviations constitute vectors $\left(u_{i}, v_{i}\right)$ on a discrete grid $\left(x_{i}, y_{i}\right)$. The deviations represent the sum of $(a)$ variations in tow positions that arose during weaving due to irregularity in the motions of the loom machinery and $(b)$ deformation fields created when the manufactured fabric was inadvertently loaded during handling.

Analysis of the deviations measured on the specimen in Figure $4 a$ revealed variations over a range of spatial wavelengths. The largest-amplitude deviations are associated with wavelengths larger than the total specimen dimensions. Shorter-wavelength variations are also present but have smaller amplitude, and their characterization therefore requires the long-wavelength deviations to be filtered out. This can be accomplished by forming spatial derivatives of the deviations (75).

Figure $4 \boldsymbol{b}$ shows spatial derivatives of the deviation component $v_{i}$ computed as finite differences and then converted into continuous fields by interpolation. The derivative $\delta_{y y}=\delta v_{i} / \delta y$ measures

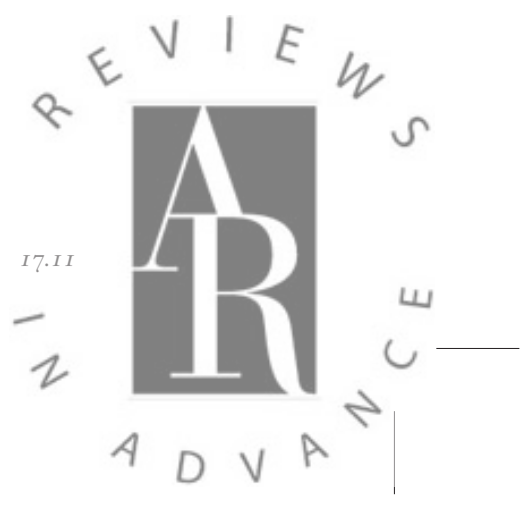


fluctuations in relative tow spacing or packing density of the warp tows; an analogous derivative $\delta_{x x}=\delta u_{i} / \delta x$ does the same for weft tows. The derivative $\delta_{x y}=\left(\delta u_{i} / \delta y+\delta v_{i} / \delta x\right) / 2$ can also be computed and measures the angle between the warp and weft tows (75).

A virtual specimen should incorporate deviations created during manufacture, because these are intrinsic to the material, while excluding deviations that are likely to have arisen due to handling, because these are the response of the material to loads and would therefore be predicted by simulations, rather than being built into the specimen being simulated. Therefore, statistical analysis of the data in Figure 4 focuses on the deviations in tow packing density, $\delta_{x x}$ and $\delta_{y y}$. Large-amplitude, long-wavelength trends in $\delta_{x x}, \delta_{y y}$, and $\delta_{x y}$ are ignored because they are believed to be caused by handling (75); short-range variations in $\delta_{x y}$ appear implicitly in the analysis of $\delta_{x x}$ and $\delta_{y y}(75)$.

As when characteristics are analyzed at the tow scale (Section 2.1), the most effective summary of the characteristics of long-range deviations takes advantage of the symmetry of the textile. In particular, both the deviations $\delta_{x x}$ and $\delta_{y y}$ vary rapidly in one direction and slowly in the other. An attractive strategy for characterizing $\delta_{y y}$, for example, is first to analyze the spectral content of $\delta_{y y}$ along lines of constant $x$ by Fourier analysis (Figure $4 \boldsymbol{b}$ ). When carried out for all available values of $x$, the amplitudes and phases of each Fourier component are stochastic and typically follow simply characterized distributions. The slow variations of $\delta_{y y}$ in the $x$-direction can then be characterized by determining a correlation length for the amplitude and phase of each Fourier component such that two points separated by more than this length have amplitudes and phases that are statistically independent of one another.

In this strategy, the complete characterization of $\delta_{x x}$ and $\delta_{y y}$, which quantify the variations in packing density of tows, is recorded as the following standard set of statistics:

$$
D_{\delta}=\left\{V_{k}^{\text {(warp) }}, V_{k}^{\text {(weft) }}, \quad k=1, \ldots, N_{\delta}\right\}, \quad 5 \mathrm{a} .
$$

where

$$
V_{k}=\left\{\left\langle A_{k}\right\rangle, \sigma_{k}, \zeta_{k}^{(A)}, \zeta_{k}^{(\varphi)}\right\},
$$

with $k$ denoting one of $N_{\delta}$ stochastic Fourier coefficients whose amplitudes follow a normal distribution with mean $\left\langle A_{k}\right\rangle$, RMSD $\sigma_{k}$, and correlation length $\zeta_{k}^{(A)}$ and whose phases are uniformly distributed over $[0,2 \pi]$ with deviations that vary with correlation length $\zeta_{k}^{(\phi)}(75)$.

For the particular material of Figure $4, \delta_{x x}$ and $\delta_{y y}$ reveal alternating bands of high and low relative packing density, with fluctuations of magnitude $\sim 1 \%$ for the weft tows $\left(\delta_{x x}\right)$ and $\sim 3 \%$ for the warp tows $\left(\delta_{y y}\right)$. A band of either high or low packing density for weft tows typically contains 30 or more weft tows. For warp tows, the bands are somewhat narrower, typically $3-5$ tows. A variation in packing density is sustained along the direction of the tow for a distance (related to $\zeta_{k}^{(A)}$ and $\zeta_{k}^{(\varphi)}$ ) of typically $100 \mathrm{~mm}$ for weft tows and $50 \mathrm{~mm}$ for warp tows (75). Interestingly, data obtained by similar experimental analysis of a $2 \mathrm{D}$ twill weave show very similar qualitative variations in tow packing density (76).

By sampling specimens much larger than the unit cell size, digital image analysis reveals statistical information that cannot be discerned in the analysis of Section 2.1, which is based on $\mu \mathrm{CT}$ data. In particular, the in-plane deviations of either weft or warp tows in the $\mu \mathrm{CT}$ data are not correlated between different tows over distances comparable to a single unit cell (9), whereas the large-scale data reveal that the deviations are indeed correlated over distances larger than the unit cell. These deductions are not contradictory: The longer-wavelength correlations between different tows would not be expected to emerge from data samples of a single unit cell, because in such a small volume the deviations discerned in Figure 4 would be approximately the same for all tows and would therefore not be detected as deviations (they would simply imply a shift in the

Cox et al. 
spatial origin). Thus, the $\mu \mathrm{CT}$ and digital image analyses are complementary; the $\mu \mathrm{CT}$ analysis yields details of deviations of tow loci and shapes at scales equal to or less than the unit cell size, and the digital image analysis yields deviations with wavelength exceeding the unit cell size.

\section{VIRTUAL-SPECIMEN GENERATION}

The next step in assembling a virtual test is to formulate a reconstruction algorithm or generator that creates stochastic virtual specimens whose statistics match those of the image data. A successful generator must match the target experimental statistics, such as the data sets of Equations 4 or 5 . Such data sets calibrate the desired generator.

The art of formulating reconstruction algorithms has a long history in image and signal processing (77-79). Applications to material science have mostly addressed polycrystalline materials (e.g., References 5, 80-84). Textiles contrast with alloys in that they comprise long, continuousfiber bundles of essentially infinite aspect ratio and interlaced in complex but systematic patterns. Their spatial arrangement calls for specialized algorithms that are quite different from algorithms developed for statistically isotropic multiphase materials $(5,81,85-87)$. The same anisotropy that affects the relative richness of data on normal sections and along the fiber direction (Section 3 ) influences the rational choice of reconstruction algorithm.

A number of methods for generating solid models of textile reinforcement have been presented over the past several decades. The simplest of these methods assume simple shapes for individual tows, e.g., tow surfaces consisting of a combination of flat segments where two tows contact one another, and curved segments elsewhere. The most advanced of such methods approximately match the assumed shapes to sectional images of tows, but without analyzing stochastic deviations $(73,88)$. Other researchers have sought to generate tow shapes close to those in experimental specimens via simulations of the mechanics of tow and preform deformation (8, 43, 89-94).

The advances described here follow an empirical approach to virtual-specimen generation: The generator algorithm seeks only to match statistical data from experiment. Models of the deformation mechanics of fiber tows or preforms, which have been the basis for most other attempts to generate realistic geometric models of textiles, are not used. There is therefore no risk of errors flowing from uncertainty either in the constitutive laws assumed to characterize such deformation or in misrepresentation of the conditions during which preform fabrication or handling is carried out, including loading boundary conditions or the presence or absence of lubricating agents. The preform is analyzed as it is, in its final disposition.

However, an empirical approach has the disadvantage that the effects of changing processing conditions cannot be predicted other than by interpolating known cases. Physically based predictions are the province of mechanical models. An attractive future development will link mechanical modeling of preform deformation to empirical statistics, using the rich data content of detailed 3D measurements to calibrate and validate the mechanical model. The empirical algorithms described here may remain useful tools within the linked supermodel.

\subsection{Generating Unit Cell-Scale Characteristics}

In the empirical approach to virtual-specimen generation, tow shapes and their deviations at the unit cell scale are generated by using the standard set of statistics of Equation 4. The simplest generator exploits the linear continuity of tows in the textile: A Markov chain algorithm generates fluctuations in any tow cross-sectional characteristic by marching systematically along the tow's length (74). The key element of the Markov chain is the probability transition matrix (PTM), which determines the deviation of any characteristic at any point on the tow given the deviation

www.annualreviews.org • Stochastic Virtual Tests

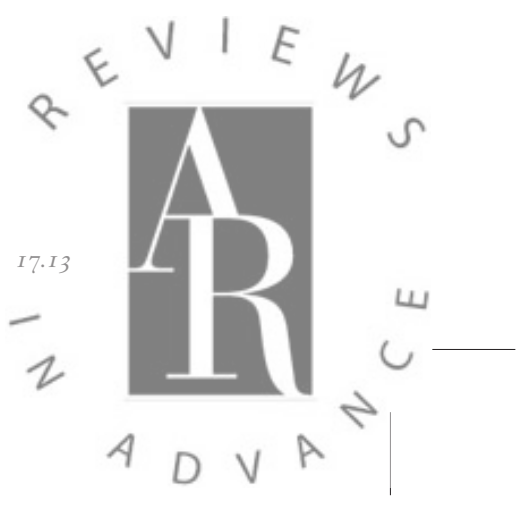




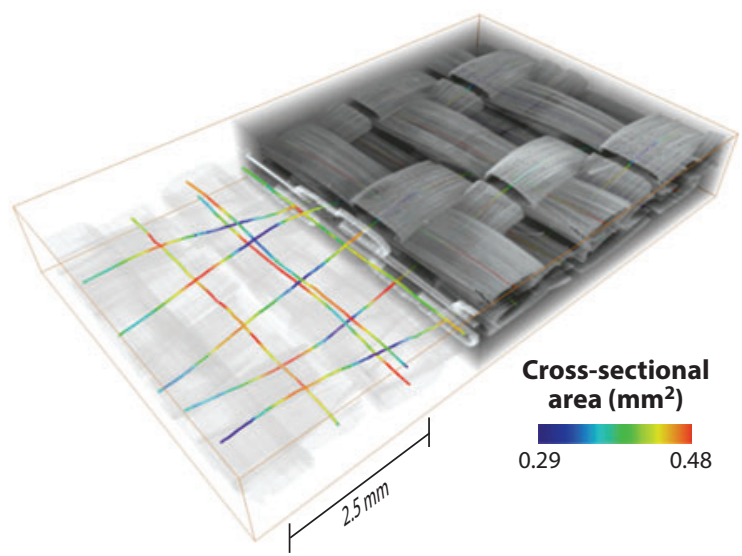

Figure 5

A $\mu C T$ image yields statistics that are matched by generated virtual specimens. The generated tow structure at the left represents tows by $1 \mathrm{D}$ loci, suitable for use in the Binary Model.

at a prior point. Whether the two deviations are strongly or weakly correlated depends on how the separation of the points compares with the correlation length for that characteristic.

The Markov chain algorithm is implemented within a Monte Carlo scheme. An instantiation $\mu_{1}$ of any deviant quantity $\mu$ at the first grid point on a tow is determined by choosing a uniform random number and then passing that number through a rescaling operation that maps it onto the prescribed distribution of $\mu$. Given $\mu_{1}$, a second random number is chosen and rescaled through the PTM of the Markov chain to generate the value $\mu_{2}$ for the second grid point. The latter step is repeated along the entire tow. Details of the implementation appear in References 74 and 95.

3.1.1. Virtual specimens for the Binary Model. Virtual specimens with 1D representations of the textile reinforcement can be generated by applying the Markov chain algorithm only to the tow center-of-mass coordinates, ignoring the other characteristics of tow cross sections. The resulting 1D tow models (Figure 5) can be incorporated into the Binary Model for analyzing the mechanics of textile composites, by combining them with solid 3D elements that create the external shape of the composite and carry constitutive properties representative of the composite matrix $(74,96)$. The Binary Model is the lowest-order computational representation that retains the interlacing pattern of the discrete tows in a textile. When the Binary Model formulation is used in conjunction with gauge-averaging methods, i.e., by averaging predicted strains over gauge lengths comparable to a tow width, mesh-independent predictions of local strain variations are obtained (97). The predictions correlate well with strength data for textiles in cases in which failure does not involve significant damage progression (i.e., cases in which failure is quasi-brittle) (98). Extension to ceramic matrix composites with a highly nonlinear matrix response has also been demonstrated (99). Some of the earliest studies of how the stochastic variance in textile geometry affects engineering material properties, replicating the six steps of a virtual test laid out in Section 1, were carried out by using the Binary Model for polymer matrix textile composites with stochastic tow geometry (100).

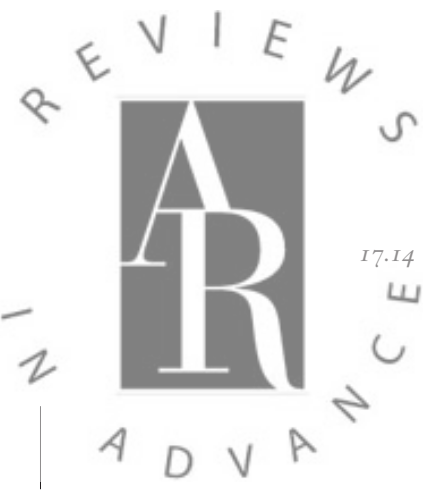

3.1.2. Solid tow representations. The same Markov chain algorithm, when applied to each of the cross-sectional characteristics listed in Equation 2 and calibrated by the corresponding measured RMSD and correlation length, can also be used to generate solid, 3D tow representations.

Cox et al. 


\begin{tabular}{|l|c|c|c|c|c|c|}
\hline & $m=1$ & $m=2$ & $m=3$ & $m=1^{\prime}$ & $m=2^{\prime}$ & \multirow{3}{*}{} \\
\hline$n=2$ & Indet & & & & \\
\hline$n=3$ & Indet & Indet & & & & \\
\hline$n=1^{\prime}$ & $Z_{m} \geq Z_{n}$ & $Z_{m} \leq Z_{n}$ & $Z_{m} \geq Z_{n}$ & & & \\
\hline$n=2^{\prime}$ & $Z_{m} \leq Z_{n}$ & $Z_{m} \geq Z_{n}$ & $Z_{m} \leq Z_{n}$ & Indet & \\
\cline { 1 - 4 } $3^{\prime}$ & $Z_{m} \geq Z_{n}$ & $Z_{m} \leq Z_{n}$ & $Z_{m} \geq Z_{n}$ & Indet & Indet & \\
\end{tabular}

b

\begin{tabular}{|c|c|c|c|c|c|c|}
\hline & $m=1$ & $m=2$ & $m=3$ & $m=1^{\prime}$ & $m=2^{\prime}$ & \multirow{6}{*}{ Warp $\underset{\text { axis }}{\rho}$} \\
\hline$n=2$ & $\rho_{m} \leq \rho_{n}$ & & & & & \\
\hline$n=3$ & $\rho_{m} \leq \rho_{n}$ & $\rho_{m} \leq \rho_{n}$ & & & & \\
\hline$n=1^{\prime}$ & Indet & Indet & Indet & & & \\
\hline$n=2^{\prime}$ & Indet & Indet & Indet & $\rho_{m} \leq \rho_{n}$ & & \\
\hline$n=3^{\prime}$ & Indet & Indet & Indet & $\rho_{m} \leq \rho_{n}$ & $\rho_{m} \leq \rho_{n}$ & \\
\hline
\end{tabular}

Figure 6

Topology of a plain weave. (a) $z$-Ordering matrix (ordering in through-thickness direction) for the three warp and three weft tows shown at the right. (b) $\rho$-Ordering matrix (ordering in plane) for the same segment of fabric. The axes to which orderings refer are shown in the inset figures. $Z_{m}$ denotes the domain of tow $m$ in the $z$-direction, $Z_{m} \leq Z_{n}$ indicates that the domain of tow $m$ lies beneath that of tow $n$, indet indicates that the ordering is indeterminate, and $m$ and $n$ are dummy indices referring to the tow numbering in the figures at the right.

The 3D tow representation problem is considerably more difficult than the $1 \mathrm{D}$ tow representation problem. When 3D tow shapes are generated, random deviations commonly cause interpenetrations between tows after intermediate steps in the generator algorithm and occasionally cause part of one tow to appear completely on the wrong side of another tow.

The question of how to remove interpenetrations between tows when generating textile models with realistic tow shapes is a long-standing challenge (101). Prior methods that have achieved the greatest realism used mechanics-based algorithms, combining deformation simulations with methods of tracking the contact surfaces between tows. The difficult nonlinear problem of tracking contact surfaces has been approached by geometrical contact models [which can be very complex (88)], simplified rules combined with FEM computations (101), or deformation computations acting alone (93).

An alternative method, which is fail-safe and computationally rapid, invokes the topological ordering rules that define the textile architecture (95). For each pair of tows, the rules prescribe which tow should lie under or over the other, if the tows are orthogonal, or which should lie to the right or left, if they are nominally parallel (Figure 6). The topological rules correct errors without ambiguity. Topological algebras appeared in textile science a quarter of a century ago and recently became a key part of generating textile models (94, 102-105). Correcting ordering errors during virtual-specimen generation by invoking formally tabulated topological ordering rules leads to very robust methods that can deal with arbitrary errors. Illustrations for virtual specimens of arbitrary size with stochastic tow shape variations (95), as well as models of nonstochastic single unit cells with periodic boundary conditions (73), have appeared.

The algorithms for generating $3 \mathrm{D}$ models also include geometrical rules for adjusting the shape and smoothness of the generated virtual specimens to match the data from imaged specimens. Alternative rules, still used in conjunction with topological ordering rules, could represent the deformation of tow shapes, e.g., deformation due to imposed surface displacement conditions

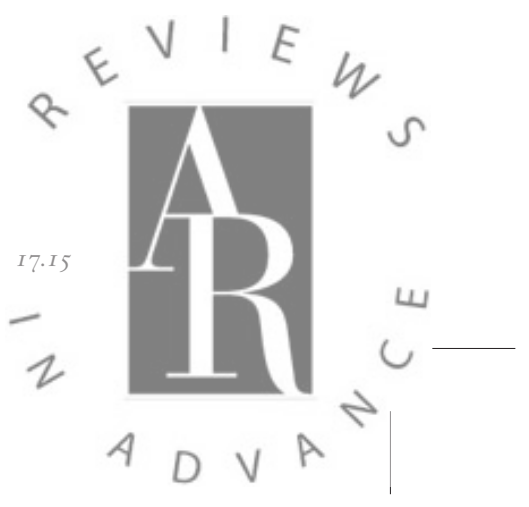



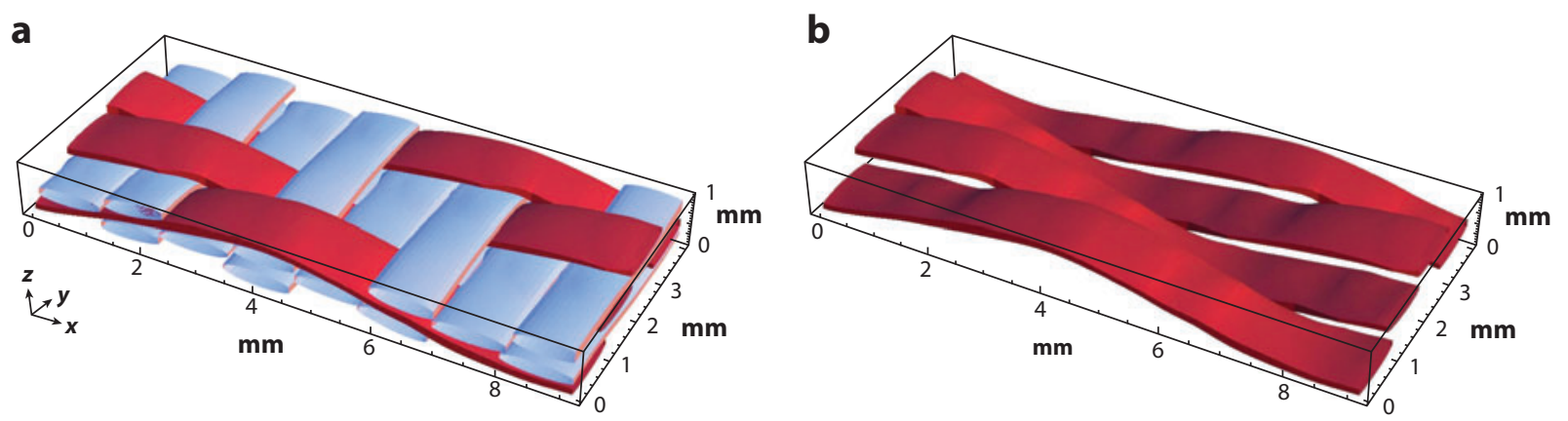

Figure 7

The textile reinforcement of a unit cell that forms part of one virtual specimen generated with $3 \mathrm{D}$ tow representations by using the Markov chain method: (a) warp and weft tows together and $(b)$ warp tows only. Tow shapes possess a combination of nonstochastic periodic variations (e.g., wave shape of tow loci) and nonperiodic stochastic deviations.

(molding). A model that changed tow shapes to satisfy boundary conditions and that was based on assumed rigid/perfectly plastic deformation, which is a fair approximation for fiber bundles under transverse shear (106), could be executed without detailed stress analysis. This model would form the simplest possible fusion of empirical and mechanics-based methods.

Figure 7 shows a typical unit cell from a virtual specimen generated with data from the specimen of Figure 3. The virtual specimen consists at this stage of tows alone, along with matrix incorporated in the tows' interiors or as thin surface layers on the tows. This is representative of textile composites used in applications in which contacting gas flow is turbulent, e.g., rocket nozzles. In applications to hypersonic engines, in contrast, the desired laminar flow necessitates that matrix be added to the tow structure to build up a smooth surface. Representing such matrix requires augmentation of the virtual specimen.

3.1.3. Validation of the statistics of virtual specimens. Correcting ordering errors can shift the generated statistics away from the target experimental statistics. However, the shifts can be easily corrected by perturbing the input targets for the generator. For example, if using the desired target value $\sigma=\sigma_{0}$ for some parameter as the input value leads to virtual specimens with $\sigma=$ $\sigma_{0}+\delta \sigma$, rerunning with $\sigma=\sigma_{0}-\delta \sigma$ as the input leads to greatly improved matching in a single iteration (95).

Validation of the fidelity of the empirical 3D generator is partially illustrated by the comparison in Figure 8, which shows that the surface topography of a generated specimen matches the systematic trends measured by digital image correlation (DIC) (107). The small differences in surface height between the experimental data and the virtual specimen (variations in color for similar tows) reflect stochastic variations in the shape and position of tows; the cases shown in Figure 8 are different instantiations of stochastic quantities. Quantitative validation of the generated stochastic variations of tow position and cross-sectional shape can also be found in Reference 95.

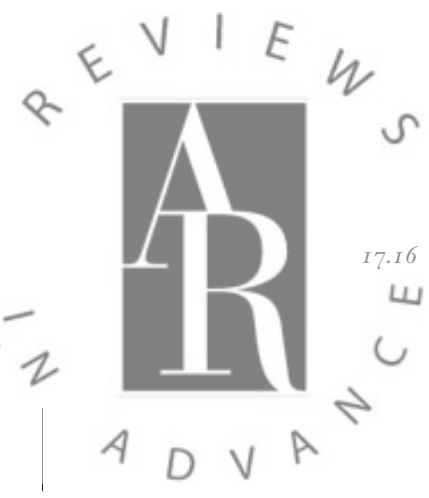

\subsection{Generating Large-Scale Characteristics}

In the empirical approach, the standard set of statistical data proposed for large-scale deviations is the set of Fourier statistics of Equation 5. Virtual specimens with the same statistics can be generated by a Monte Carlo process that combines inverse Fourier transforms for generating

Cox et al. 

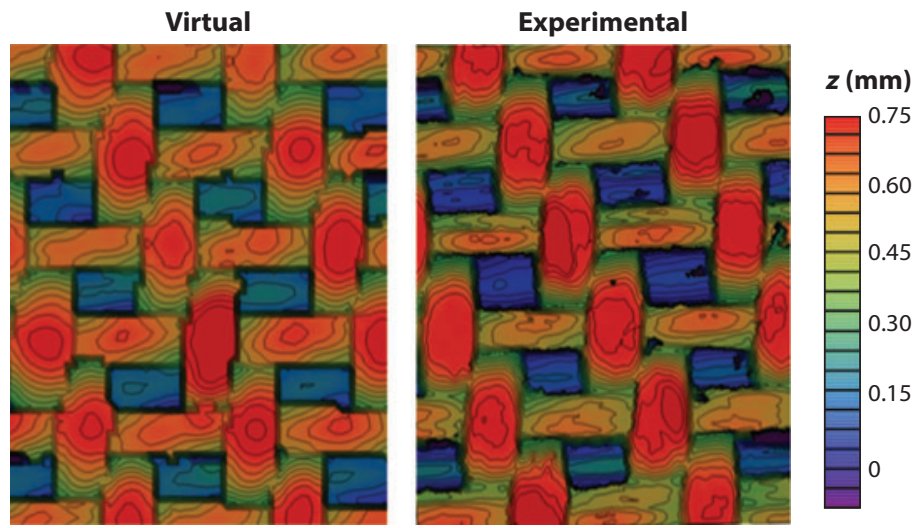

Figure 8

The surface topography obtained from digital image correlation measurements and a corresponding virtual sample generated by using $\mu \mathrm{CT}$ data; details of this analysis can be found in Reference 107.

variations in the direction normal to tows, where they are most rapid, and rules based on simple Markov processes to generate variations along the fiber direction, where changes are gradual.

The algorithm proceeds analogously to that used to generate successive cross sections of a single tow. At each grid point along the tow direction, tow packing density deviations are generated for all tows in a genus across the specimen. For example, to generate density deviations for weft tows, the algorithm begins at the lowest value $x_{i}$ of coordinate $x$ in the specimen (in the coordinates of Figure 4). Random values are chosen for the amplitude and phase $A_{k}$ and $\phi_{k}$ of each Fourier component $k$, weighted according to the probability density functions for that component, and used as input for an inverse discrete Fourier transform to evaluate the deviations at all grid points for that value of $x$ (Figure $4 b$ ). Given the set of amplitudes and phases at $x_{i}$, the amplitude for each Fourier component at $x_{i+1}$ is obtained by applying a Markov chain algorithm, using a PTM that was calibrated to match the data for the correlation length $\zeta_{k}^{(A)}$, while the phase of the Fourier component is obtained via a random-walk algorithm calibrated to the correlation length $\zeta_{k}^{(\varphi)}$. (The random-walk algorithm suits the problem of generating phases, which have unbounded mean when not confined to $[0,2 \pi]$ and are uniformly distributed over $[0,2 \pi]$ when truncated to reside in $[0,2 \pi]$.) The process of generating amplitudes and phases is iterated for all $x_{i}$.

Figure $4 c$ shows a typical set of tow packing density variations in a virtual specimen generated by this algorithm. The patterns of variations are similar to those measured experimentally (Figure $4 \boldsymbol{b}$ ) but are not identical, as they are a different instantiation of the random field. Reference 75 provides further details.

\subsection{Combining Statistics from Different Scales}

Statistical data for the unit cell scale and large scale (Equations 4 and 5), which emerged from different experiments, are treated above as separate problems and are summarized in distinct standard data sets. An important question is what correlations might exist between the metrics gathered for the two scales.

Analysis of experimental data for the angle interlock weave of Figure 3 revealed that correlations between deviations in tow position and deviations in tow shape are negligibly weak (9). In other words, when the packing density of tows varies over the range present in these materials,

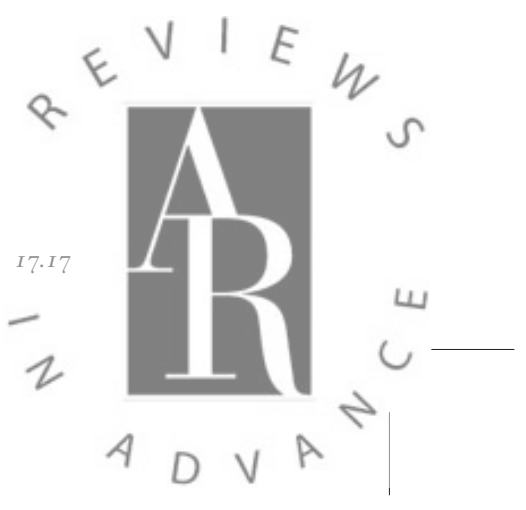


the tows shift sideways without any significant change in the deviance of various statistical metrics of shape.

The weakness of such correlations has the important consequence that, at least for this material, the unit cell-scale deviations and large-scale deviations can be assumed to be independent of each other and to be generated separately in a virtual specimen from the standard sets of Equations 4 and 5 and combined by superposition.

That deviations in packing density (the long-range metrics) and deviations in tow shape (shortrange metrics) should be uncorrelated is intuitively plausible. Consider, for example, the effect of uniformly changing the average packing density of tows across a specimen. The mean tow shape can change substantially, especially as tows are packed harder into one another, but the deviations in shape from this new mean could well remain weakly correlated with deviations in the packing density from the new mean density. Whether statistics are correlated is determined among deviations, not mean values.

However, if tows are packed tightly in some regions and kept sparse in other regions, the deviations of the tow shapes from the mean could be different in the densely and sparsely packed regions, and the correlations between long ranges and short ranges could therefore be strong. But for such an effect to be of concern in a virtual-specimen generation, the differences in packing density would evidently have to be much greater than they are in the material studied.

\section{TEST DATA: THE HIGH-TEMPERATURE CHALLENGE}

Given a sample microstructure created by the virtual-specimen generator, virtual-specimen definition is completed by specifying constitutive laws for material phases and for the nonlinear damage events that arise during failure. Simulations of failure are then formulated; in such simulations, the appearance of very general local damage events is enabled by the manner in which computational elements are defined. Key to the process are experiments that identify which local damage events should be included in simulations and the nature of the damage event: A simulation is much more likely to achieve fidelity if the form of the mechanisms it must be able to reproduce is already known. The experiments must also provide data for model calibration.

A considerable literature exists on experiments that identify local damage mechanisms in ceramic composites and monitor the evolution of such mechanisms during mechanical loading by a variety of in situ measurements. Such measurements include those via optical microscopy (108113), scanning electron microscopy (114-119), speckle interferometry (120-122), acoustic waves (123-125), and electrical resistance $(126,127)$. Most of these measurements were at room temperature; only a few studies tackled in situ measurements at high temperatures (e.g., References 114 and 128). In situ tomographic observations of deformation in an aluminum alloy have been made at temperatures as high as $555^{\circ} \mathrm{C}(129)$, but this temperature is much lower than the temperature range of interest for ceramic composites.

The experimental challenge is sharpened by the facts that the materials are to be used at very high temperature and that their behavior at high temperature cannot be assumed to be represented by their behavior at room temperature. Consider, for example, a high-temperature test followed by postmortem examination at room temperature: If a hot specimen is cooled to an experimentally convenient $25^{\circ} \mathrm{C}$ for examination, the cooling introduces thermal strains of the order of at least $0.1-0.5 \%$, depending on the composition and cooling rate, which can change the cracking patterns present.

Ideal tests for acquiring knowledge of mechanisms and constitutive data should be executed on the composite materials themselves under expected use conditions. Current applications in turbine engines or rocket nozzles, for example, present temperatures in the $1,200-1,500^{\circ} \mathrm{C}$ range,

Cox et al. 


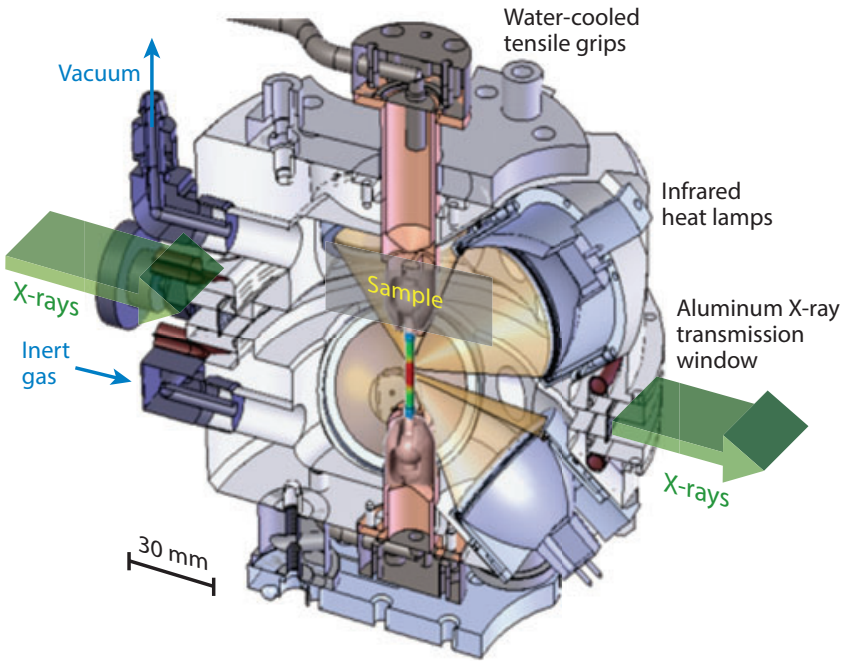

Figure 9

Sectional view of the heating chamber in a test rig designed to permit $\mathrm{X}$-ray $\mu \mathrm{CT}$ imaging during testing at high temperature. The $\mathrm{X}$-ray beam passes through a load-supporting aluminum window. The window's absorption is constant during rotations of the chamber and therefore does not impair image quality. The sample is held by water-cooled grips in the center of a sealed cell, which can be evacuated or filled with a selected gas. A hexapole arrangement of six 150-W halogen lamps and reflectors gives a spherical hot zone of $\sim 5$-mm diameter.

and even higher temperatures are sought (51-53). The tests must also have resolution comparable to the scale of the heterogeneities and damage events that control material performance. Two new experimental systems have been developed to meet this dual challenge. Their use to identify damage mechanisms is described below, whereas their use to acquire calibration data is described in the following section.

\subsection{D Imaging Using $\mu \mathrm{CT}$}

Although $\mu \mathrm{CT}$ was first demonstrated two decades ago (130), only recently have groups reported apparatus for acquiring $3 \mathrm{D} \mu \mathrm{CT}$ images of evolving damage in a ceramic composite specimen while loading in tension or compression in a high-flux synchrotron beamline. One system operates at room temperature $(131,132)$ and the other operates at temperatures of up to of $1,500-1,750^{\circ} \mathrm{C}$ in inert or oxidizing atmospheres (Figure 9) (58). The current maximum spatial resolution is $0.65 \mu \mathrm{m}$ voxel $^{-1}$, yielding rich data on microstructure down to the fiber scale, and failure mechanisms are resolved at submicrometer scales. Key data related to microcracking include spatial maps of matrix cracks and fiber breaks and opening displacements of cracks of both of these types as a function of load.

Data for a monotonic tension test of angle interlock carbon/ $\mathrm{SiC}$ woven composite specimens reveal different mechanisms of failure operating at $25^{\circ} \mathrm{C}$ and $1,750^{\circ} \mathrm{C}$ (Figure 10). At the lower resolution $\left(1.3 \mu \mathrm{m}\right.$ voxe $\left.^{-1}\right)$ used in these images, individual carbon fibers were not resolved. Nevertheless, the fiber tows are clearly distinguished from the matrix, which consists of a thin, brighter layer of chemical vapor infiltration (CVI) SiC coating each tow and a polymer-derived $\mathrm{SiC}$ filling the remaining occupied space. During initial loading at both temperatures, cracks formed in the matrix normal to the loading direction at positions where the matrix lay over a transverse

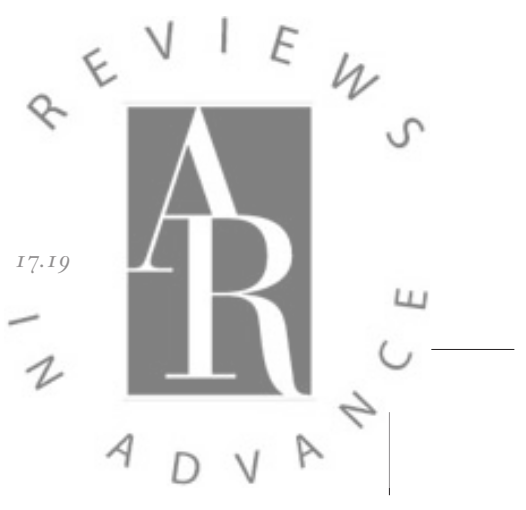



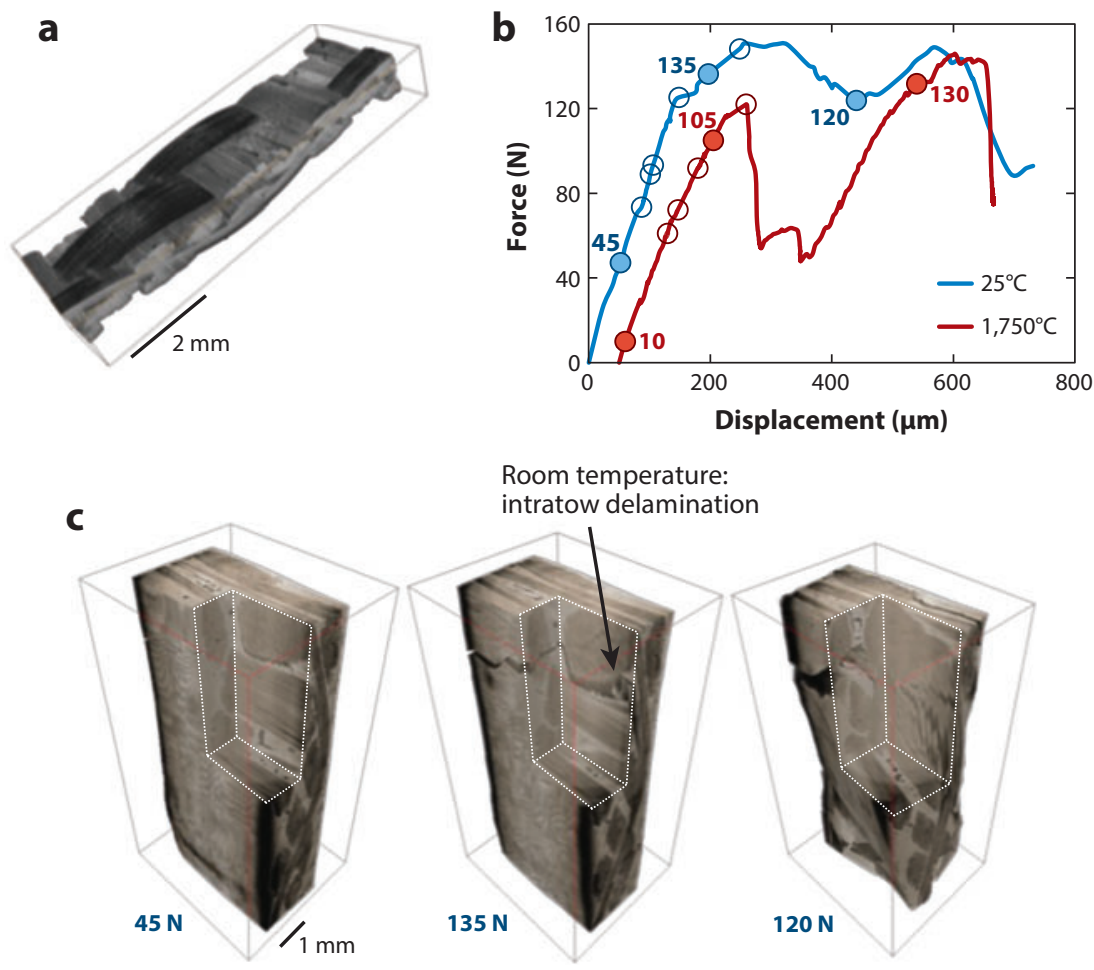

Room temperature:

d

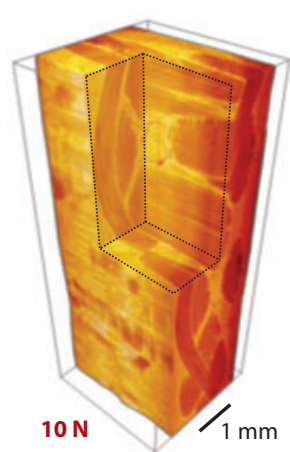
intratow delamination

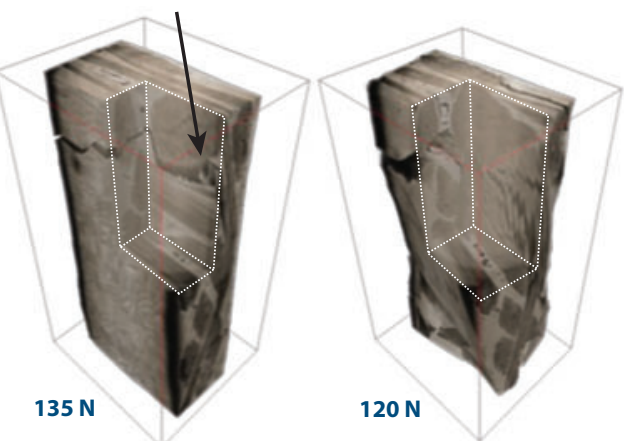

High temperature: interstitial matrix damage
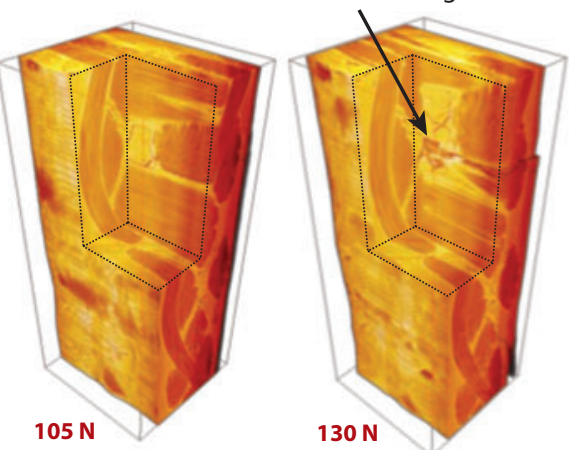

Figure 10

Internal damage in a carbon/ $\mathrm{SiC}$ composite with textile-based carbon fiber reinforcements under tensile load at $25^{\circ} \mathrm{C}$ and at $1,750^{\circ} \mathrm{C}$. (a) A $\mu \mathrm{CT}$ image illustrates the architecture of the woven fiber tows within the test samples, which were fabricated with minimal matrix. $(b)$ Force-displacement curves showing loads at which $\mu \mathrm{CT}$ data were collected. The red curve is offset by $60 \mu \mathrm{m}$ for clarity. Open circles indicate $\mu \mathrm{CT}$ data acquired at that load; blue and red solid circles indicate loads corresponding to images in panels $c$ and $d$, respectively. $(c, d) \mu \mathrm{CT}$ images showing the development of damage in specimens tested $(c)$ at room

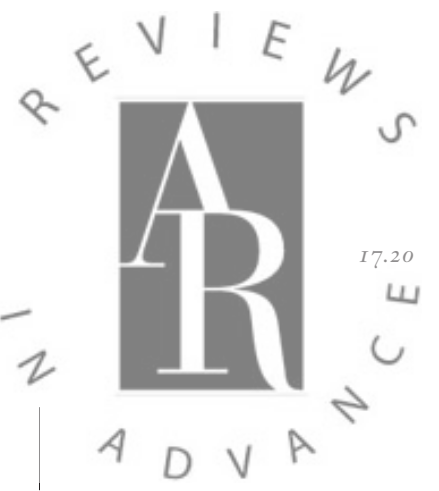
temperature and $(d)$ at $1,600^{\circ} \mathrm{C}$. 
fiber tow. With increasing load, the cracks grew through the transverse tows until they met an underlying axial tow (at loads in the range of $\sim 40$ to $70 \mathrm{~N}$ ), at which they were deflected. At $25^{\circ} \mathrm{C}$, this deflection involved the formation of multiple splitting cracks (Figure 10c), which progressed incrementally along the centers of the axial tows as the load increased to the peak value of $150 \mathrm{~N}$. Above $1,600^{\circ} \mathrm{C}$, the deflection of the crack at each tow involved a single crack that grew along the edges of the axial fiber tows as the load increased to $120 \mathrm{~N}$ (Figure $\mathbf{1 0 d}$ ), whereupon there was a large load drop (Figure 10b).

By influencing the access of ambient gas to the internal reinforcing fibers, details in crack paths could potentially have a large effect on high-temperature oxidation damage. The ability of a simulation to replicate the differences observed in crack patterns at room temperature and high temperature is therefore a key validation test.

\subsection{Digital Image Correlation}

Although DIC has a long history for room-temperature studies (133-137), direct optical imaging of surfaces at very high temperatures (up to $1,500^{\circ} \mathrm{C}$ ) is complicated by the high intensity of visible wavelengths emitted by both the specimen and hot testing components. A newly developed system overcomes light noise by illuminating with 10 -W blue LED assemblies, whose wavelength of 440$460 \mathrm{~nm}$ is separated from the most intense black-body radiation, and by using band-pass filters to tune charge-coupled-device cameras to the wavelengths of the LED sources (138). Suitable lenses and judicious placement of the LEDs with respect to the target surface yield a viable viewing region several centimeters in diameter. The optical system is combined with a laser facility that simultaneously enables heating of the specimen and imaging, including measurements of strain fields via DIC. The DIC measurements require the use of high-temperature ceramic paints to create a stable speckle pattern. Finally, an air knife minimizes distortions due to thermal turbulence by thoroughly mixing air in the line of sight of the imaging system.

Figure 11 shows representative results for the two in-plane thermal strain components measured at $1,026^{\circ} \mathrm{C}$ with submillimeter resolution in the woven carbon/ $\mathrm{SiC}$ composite of Figure 3, along with surface topography data, which reflect the fiber weave (139). Superimposed onto the strain maps are the approximate locations of the boundaries of the surface weft and the warp tows. Local variations of the thermal strain components correlate with the surface tow positions. Within
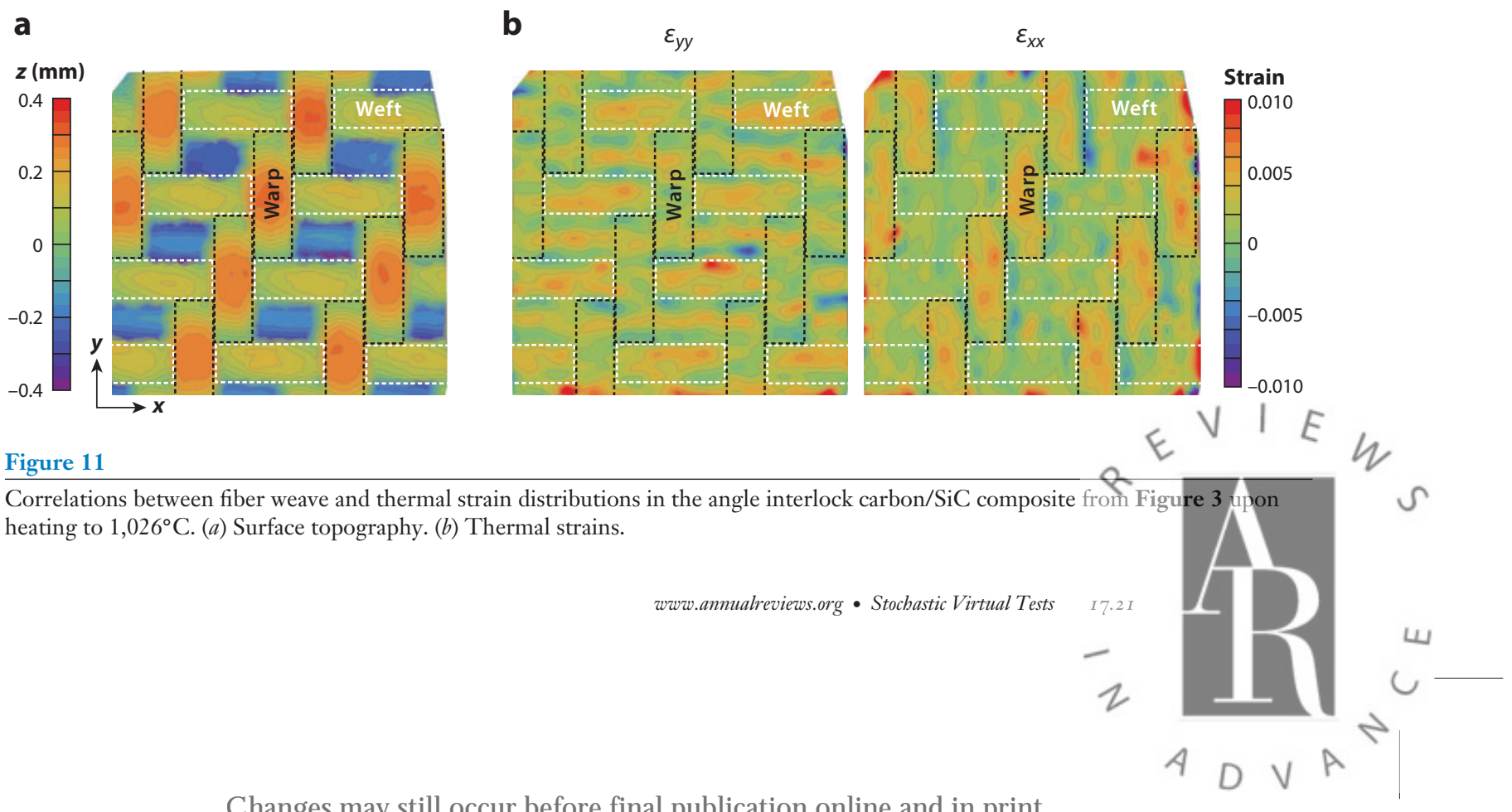
both the warp tow and the weft tow segments, the thermal strain is smaller in the direction parallel to the fibers $(\sim 0.002)$ than in the transverse direction $(\sim 0.005)$. This finding is qualitatively consistent with expectations: The thermal expansion of the carbon fibers is highly anisotropic (negative in the fiber direction and $\sim 9 \times 10^{-6} \mathrm{~K}^{-1}$ in the transverse direction), making the carbon/SiC tow segments correspondingly anisotropic. The data in Figure 11 were combined with iterative FEM models to calibrate the thermal and elastic constitutive properties of the tow segments for use in subsequent virtual-test analysis of composites with fully infiltrated matrices (Section 5.3).

\section{CALIBRATION OF MATERIAL PROPERTIES AND FRACTURE LAWS}

The properties (e.g., strength and thermal conductivity) of a composite's constituents and their interfaces are generally unknown at high temperature and must be determined by experiment. The required properties include those of pristine constituent materials and those needed to model damage events, such as cracks and oxidation. Constitutive laws for cracks consist of tractiondisplacement relations that describe the nonlinear fracture process zones and Biot numbers that describe the impact of a crack on heat transport. Other constitutive laws might describe, for example, oxygen diffusion or creep.

Establishing test procedures for calibrating virtual specimens is a major part of developing virtual-test systems. As finer scales are included in hierarchical models, standard engineering tests are no longer applicable; there is a need for experiments that probe deeper into the material, yet are sufficiently reliable and repeatable to be used as calibration procedures. Research on this general topic is likely to continue for many years.

\subsection{The Special Challenges of Ceramic Composites}

Polymer composites are formed by very mature engineering processes, which yield nearly perfectly dense (porosity-free) and homogeneous matrices. The elasticity of the matrix can be deduced from data for composites by applying well-established micromechanical models. The inferred matrix elasticity is generally not noticeably different from the elasticity of neat specimens of the polymer cured in the same cycle. Therefore, one can regard the elasticity of both the fibers and the matrix as known a priori, and accurate predictions of the composite elasticity ensue $(66,100,140-142)$.

A ceramic matrix, in contrast, is formed by processes that commonly create substantial porosity and a microstructure that is not the same as that found in monolithic specimens of nominally the same material. The matrix material is peculiar to the geometrical environment (the interstices of fiber bundles) and to the infiltration method (e.g., CVI, reactive melt infiltration, or polymer precursor) that govern its formation; the material's elasticity and thermal expansion can be very different from those of nominally equivalent monolithic specimens. The situation is further complicated by the presence of fiber coatings, essential for damage-tolerant behavior, whose thermoelastic properties are again uncertain. In one study, SiC matrices formed by a combination of CVI and polymer precursor reaction around coated carbon fibers were deduced by analysis of composite elasticity to be more than an order of magnitude more compliant than monolithic $\mathrm{SiC}$ (122).

The case of ceramic composites can be further complicated by the presence of matrix microcracks induced by thermal strains during cooldown from high-temperature processing. Figure $12 a$ shows microcracks in a carbon fiber/SiC matrix composite similar to the material of Figure 3 but in which additional SiC matrix was added to fill out the space between tows and to form flat specimen surfaces. Examination of differential displacement fields by DIC shows that these cracks were present from very low applied loads (Figure $\mathbf{1 2 b , c}$ ); they were probably present in the pristine composite.

Cox et al. 
a

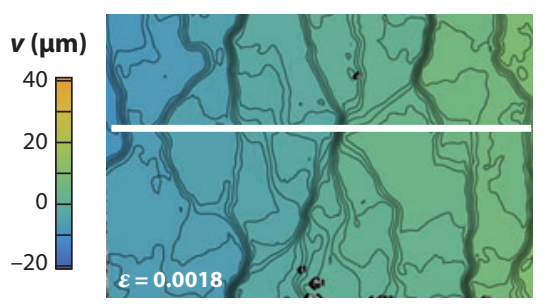

b

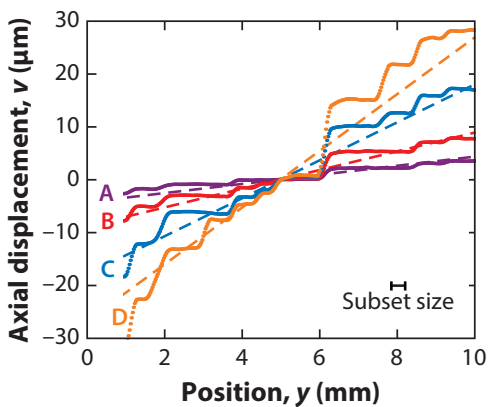

C

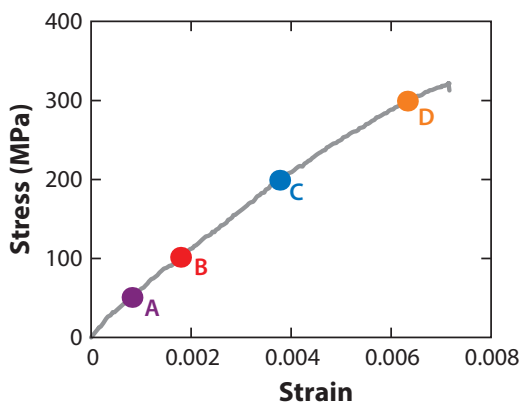

Figure 12

(a) In a composite in which the matrix fills out space to a flat surface, microcracks seen on the surface by digital image correlation correlate approximately with the textile architecture. (b) Displacement fields along the scan line shown by the white line in panel $a$ at points A, B, C, and D in the stress-displacement curve of panel $c$ show that displacement discontinuities are present at the lowest loads, implying that microcracks are present in the composite as processed.

Processing microcracks may not always be present in a ceramic matrix composite. Some carbon/SiC composites formed by repeated polymer precursor infiltration processing have high levels of hermeticity and minimal microcracking before loading $(49,121)$, and $\mathrm{SiC} / \mathrm{SiC}$ composites formed by melt infiltration tend to be microcrack free (143). Nevertheless, whether present initially or induced by loading, microcracking causes nonlinear macroscopic behavior and complicates property calibration. Because ceramic matrices tend to be much stiffer than polymer matrices, matrix cracks have a much greater relative effect in ceramic composites than in polymer composites.

\subsection{Elasticity}

In the current status of ceramic processing science, the elasticity of a ceramic matrix must be inferred from a test on a composite specimen. The initial linear elasticity of the matrix-impregnated fiber tows in the carbon/ $\mathrm{SiC}$ material of Figure 3 was calibrated by fitting test data (107). With this step completed, the elasticity of the porous and initially microcracked matrix region, which was not known a priori, could be separated out by applying micromechanical models of unidirectionalfiber/matrix composites and by using the known fiber elasticity. An analogous approach extends calibration into the nonlinear regime, in which additional matrix cracking occurs. Numerous examples of the calibration of the nonlinear matrix modulus from composite data are available $(99,121,122,144-146)$.

\subsection{Thermal Expansion}

In a recent analysis, the anisotropic thermal expansion coefficients for impregnated tows in the carbon/SiC material of Figure 3 were calibrated by using the DIC data in Figure $\mathbf{1 1}$ in combination with iterative FEM analysis of a virtual-test specimen (139). The axial and transverse tow-scale expansion coefficients were determined to be $1.6 \times 10^{-6} \mathrm{~K}^{-1}$ and $6.5 \times 10^{-6} \mathrm{~K}^{-1}$, respectively. The calibrated transverse expansion coefficient was close to the value predicted by using a micromechanical model with best estimates for properties of the fiber and infiltrated fiber coatings and matrix. However, the calibrated axial expansion coefficient was approximately

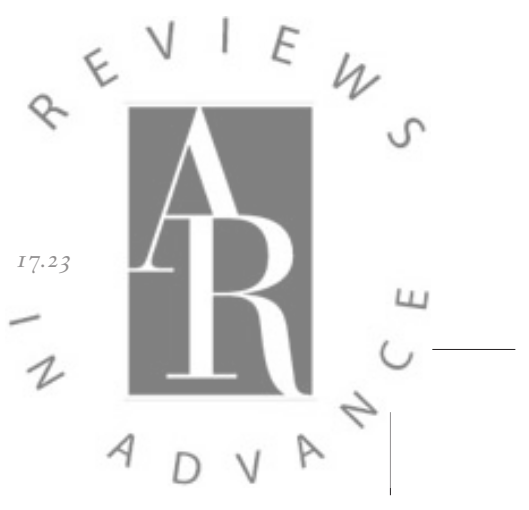


one-half of the predicted value. The difference was attributed to the influence of the preexisting matrix microcracks in Figure $12 a$.

\subsection{Damage}

Composite materials fail by the interaction of multiple damage mechanisms acting at a variety of scales. Simulations of failure in laminated polymer composites have successfully predicted the evolution of quite complex systems of interacting splitting, delamination, and transverse ply cracks. In polymer composites, multiscale mechanisms are present, so an effective ply-level model (i.e., a model using homogenized plies) must include both discrete representations of ply-scale cracks and continuum plasticity to represent damage occurring at the fiber scale (24).

In textile ceramic matrix composites, microplasticity is minimal (except at very high temperatures), and multiscale effects consist of microcracking that occurs at different scales, for example, breaks of an individual fiber or failure of its interface with the matrix, matrix cracks of size commensurate with the width of a tow, and macroscopic cracks spanning many tows. In a textile architecture, these crack systems evolve in spatially complex patterns, such as those seen in the $\mu \mathrm{CT}$ images of Figure $\mathbf{1 0}$ and in the DIC image of Figure $\mathbf{1 2 b}$.

The highest-fidelity simulations of such complex cracking predict each crack in the evolving system as a discrete event. For discrete crack representations, the constitutive law that must be calibrated is the cohesive fracture law that controls the nonlinear fracture process. If cracks appear at large densities, a continuum damage model may be attractive (147-150). In this case, calibration is of a nonlinear stress-strain law for the cracked material domain, which, from the point of view of computational implementation, is similar to the nonlinear plasticity law used in polymer composites.

5.4.1. Nonlinear continuum laws. In one phenomenological approach, nonlinear continuum damage laws for ceramic composites are calibrated by using data from tension tests of unnotched specimens executed at two different orientations $(151,152)$. Stresses for other specimen configurations can be predicted accurately by using the calibrated laws, provided that changes in stress components remain proportional at any point during damage evolution, which is a reasonable assumption in many cases. The Genin-Hutchinson model supports prediction of the entire nonlinear load-displacement curve for a component up to failure. A simpler calibration procedure that focused on initial elasticity and ultimate strength successfully predicted the effect of stress concentrators on strength (99).

5.4.2. Representations of discrete cracks. The cohesive zone representation of fracture works well for polymer composites because the nonlinear fracture process zone is not too small, usually at least comparable to ply-scale gauge lengths (ply thickness or tow width) (98, 153-156). In ceramic composites, in contrast, the fracture process zone is sometimes very small; in a brittle matrix material, including materials such as CVI-formed SiC, cracks may even be atomically sharp (157).

In a sufficiently brittle constituent phase of a ceramic matrix composite, the preferred approach to simulating crack growth is to use linear elastic fracture mechanics, with propagation controlled by a crack tip stress intensity factor, $K_{\mathrm{c}}$, associated with a point representation of the crack tip. Failure initiation is governed by the statistics of defects: The local critical stress for crack growth from a defect of size $a$ varies as $K_{\mathrm{c}} / a^{1 / 2}$, with $K_{\mathrm{c}}$ the fracture toughness.

In less brittle constituent phases, when some mechanism provides sufficient nonlinearity to create a fracture process zone of significant length, representing fracture by cohesive zones rather than by point processes can also be valid (158-160). Furthermore, even for a brittle material,

Cox et al. 
a

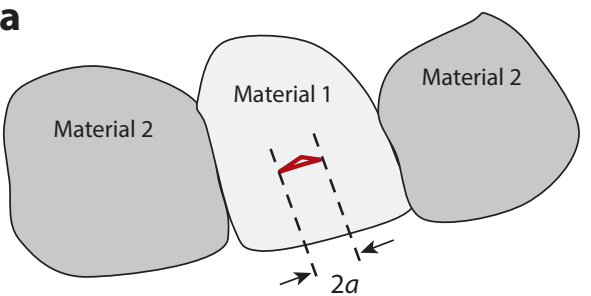

b

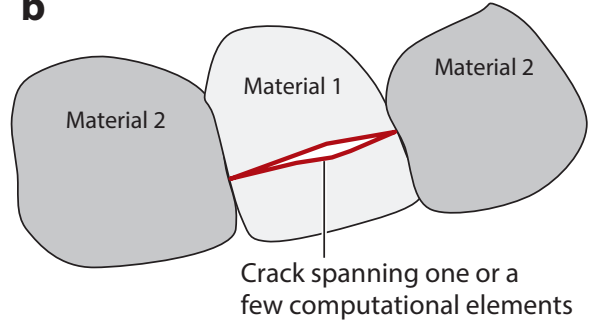

Figure 13

(a) Beginning and (b) end configurations for a simplified representation of unstable, brittle crack growth from an initial defect in a heterogeneous material.

when a crack becomes long enough, the tip process governing its advance can be represented with acceptable accuracy by a cohesive zone model, i.e., by introducing a fictitious process zone, as discussed below.

5.4.2.1. Calibration of point fracture processes. For local failure within a heterogeneous material, a simple strategy for calibrating a point fracture process is illustrated in Figure 13. The material between two heterogeneity boundaries contains a flaw of size $2 a$ (Figure 13a). The figure may represent, e.g., a matrix domain between two tows (a tow-scale problem), a ply between two ply boundaries, or matrix between two fibers (a fiber-scale problem). In a brittle material, crack growth from the flaw, once initiated, is likely to proceed unstably until the material heterogeneity imposes a barrier to further growth. Such a phase of unstable growth can be represented in a simulation first by testing whether the local stress has attained a critical value

$$
s_{\mathrm{c}}=K_{\mathrm{c}} a^{-1 / 2}
$$

and then, upon satisfaction of this criterion, by introducing a traction-free crack that immediately spans the entire interval from one heterogeneity boundary to the other (Figure 13b). Such a crack typically spans one or a few computational elements. Because the crack now has a significant size, its further growth, either by fracture of the next material phase or by growth along an interface, can be represented accurately by using cohesive fracture modeling, even if the subsequent cracking is through a brittle medium. A fictitious cohesive zone can be substituted for a point process without computational error, provided that the crack length is much greater than the process zone length. The error in computed energy release rate, which is proportional to $a$ for a brittle crack, is bounded approximately by $l_{\mathrm{c}} / a$, where $l_{\mathrm{c}}$ is the length of the fictitious cohesive zone, and is usually much less than this bound. The cohesive and point process representations are asymptotically equivalent as a crack gets larger $(159,160)$.

For the representation just described, calibration consists of determining the statistical distributions of the geometry of the material phases, the size of the defects, and the toughness $K_{\mathrm{c}}$ for the brittle material. A calibrating test would record the statistics of first cracking in composite specimens, e.g., by using the $\mu$ CT imaging or DIC apparatus described in Section 4. Pineau et al. (161) demonstrated such a calibration that deals with issues similar to those discussed here.

5.4.2.2. Calibration of cobesive laws. When sufficient nonlinearity is present in fracture, a cohesive representation is preferred. The following case study demonstrates the calibration of mixed-mode cohesive fracture laws for a ceramic matrix composite and the successful subsequent

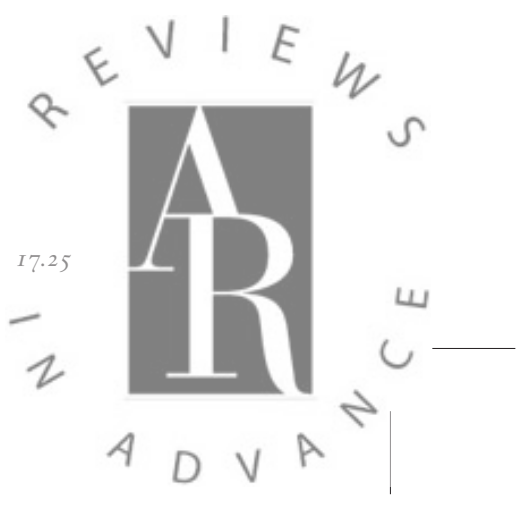


a

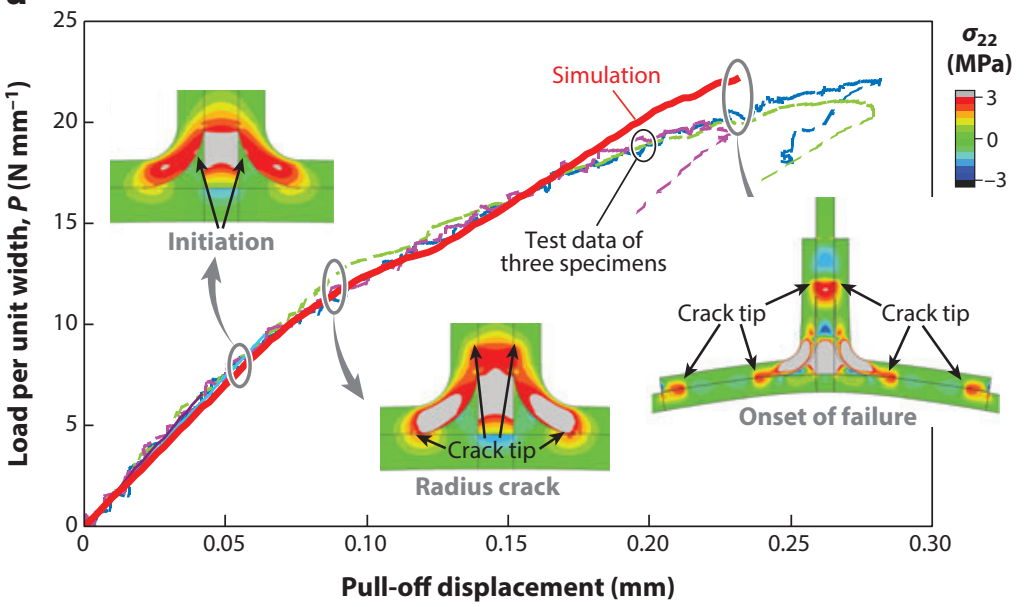

b

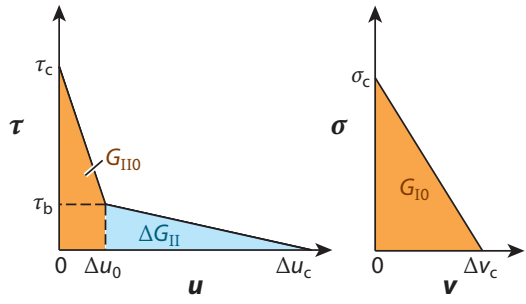

Figure 14

(a) Test data and simulations (solid red curve) of the failure of a T-stiffener. (b) Cohesive fracture models for mode II fracture (traction $\tau$ versus displacement $u$ ) and for mode I fracture (traction $\sigma$ versus displacement $v$ ) were calibrated by independent tests and were combined into a mixed-mode law for use in the simulations. Parameters $\tau_{\mathrm{c}}, \tau_{\mathrm{b}}, \Delta u_{0}, \Delta u_{\mathrm{c}}, \sigma_{\mathrm{c}}$, and $\Delta v_{\mathrm{c}}$ are the calibrated fracture properties.

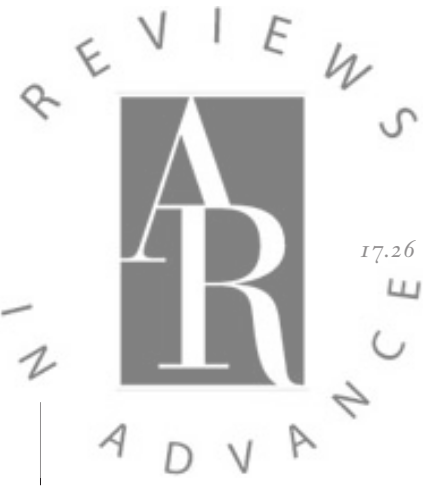

Changes may still occur before final publication online and in print no adjustable parameters.

Cox et al. prediction of the damage evolution and failure of a structural subcomponent (O. Sudre \& Q.D. Yang, unpublished work).

The subject material was a laminate of satin weave plies of $\mathrm{Nextel}^{\mathrm{TM}} 610 \mathrm{Al}_{2} \mathrm{O}_{3}$ fibers in a predominantly $\mathrm{Al}_{2} \mathrm{O}_{3}$ matrix that was formed by slurry infiltration (material supplied by COIC Inc.). The structural subcomponent was a T-stiffener comprising a flange and web joined by laminated radius elements (see insets of Figure 14a). Under tensile load, the T-stiffener failed first by delamination in the radius elements, then by stable delamination crack propagation, and finally by further delamination initiation at the termini of the radius elements adjacent to the flange.

A double-cantilever beam (DCB) test and an end-notch flexure (ENF) test were used to calibrate the matrix material for delamination fracture of different modes. The strength and toughness values measured in the DCB test were identified with the peak traction and bounded area (work of fracture) in a piecewise-linear, decreasing cohesive law for mode I fracture (Figure 14b). For mode II delamination, a four-parameter bilinear cohesive law (Figure 14b) was calibrated by varying the parameters to fit the entire load-deflection curve for the ENF test and the measured length of the fracture process zone. The zone length was determined by using the high-accuracy automated strain-mapping (HASMAP) system, an early DIC system (137). A cohesive law for any mode mix was then constructed by following a procedure that was developed for polymer adhesives (162). Material failure (loss of cohesive tractions) is governed under mixed-mode conditions by attainment of the criterion $G_{\mathrm{I}} / G_{\mathrm{I} 0}+G_{\mathrm{II}} / G_{\mathrm{II} 0}=1$, where $G_{\mathrm{I}}$ and $G_{\mathrm{II}}$ are the areas under the mode I and mode II cohesive laws, respectively, up to the current mode I and mode II displacements, whereas $G_{\mathrm{I} 0}$ and $G_{\mathrm{II} 0}$ are the total areas under the same curves (Figure 14b). This mixed-mode law has been used successfully to analyze multiple interacting fracture modes in polymer tape laminates; further details may be found in Reference 24 . With elasticity calibrated by the initial compliance in the calibrating fracture tests, simulations of the T-stiffener were fully defined, with 
The calibrated simulations correctly predict the location of the first damage (delamination in the radius), the phase of stable delamination crack growth, the initiation of delaminations adjacent to the flange, and the applied load corresponding to each of these events. The entire load-deflection curve is also correctly predicted (Figure 14), except that the simulation slightly underestimates the displacement at the highest loads; the simulation used an early formulation that permitted only certain crack systems to develop and did not capture additional delamination failures that occurred in experiments at high loads within the laminates.

Calibration in the example of Figure $\mathbf{1 4}$ could be based on standard tests because the material had a 2D laminar structure: Delaminations that governed failure occurred between sublaminates and involved local material geometry and processing conditions that were well represented by the standard test specimens. In 3D textiles, and especially for cracks at smaller scales, e.g., microcracks within a single fiber tow or microcracks between tows whose size is constrained by the textile architecture, as in a $3 \mathrm{D}$ weave, standard tests may not be representative. Another route to calibration is needed.

For tow-scale cracks whose geometry is complicated by interactions with 3D textile architecture, calibration of a high-fidelity simulation is likely to involve analysis of complex experiments using the simulation itself: Parameters within the simulation can be calibrated by fitting its predictions to the observed crack patterns, crack sizes, and crack shapes (161). In the current status of development of virtual tests, the definition of such calibration tests is made on a case-by-case basis but is encouraged by the fact that the location, size, and shape of microcracks, such as the microcracks observed in Figure 10, are a rich source of information about fracture cohesive laws (24).

5.4.2.3. Calibration of fiber-scale data. The fiber/matrix friction coupling stress, $\tau$, is a critical property in the mechanics of brittle matrix composites, controlling the stress for matrix fracture and the transition from brittle to ductile fracture (163-165). Measurement of $\tau$-which, along with the fiber volume fraction and the fiber and matrix elasticity, is a fiber-scale property-calibrates the bridging constitutive law that controls matrix cracking at the tow or ply scale.

High-temperature $\mu \mathrm{CT}$ data enable the inference of $\tau$ at different temperatures from fracture tests on composite specimens. Illustrative experiments and analysis have been carried out for minicomposites consisting of a single tow of SiC fibers consolidated by CVI with a $\mathrm{SiC}$ matrix. The required data for deducing $\tau$ are the opening displacements of matrix cracks, such as the cracks shown in the $\mu \mathrm{CT}$ image of Figure $\mathbf{1 5} \boldsymbol{a}$, as a function of the average stress in fibers, which can be deduced from the applied load. Such data can be fitted successfully by curves predicted by shear lag theory (Figure 15b), with the value of $\tau$ emerging from the best fit (58). For the material examined, the friction stress falls from approximately $2 \mathrm{MPa}$ at room temperature to $0.4 \mathrm{MPa}$ at $1,750^{\circ} \mathrm{C}$. (Details of the analysis, including accounting for idiosyncrasies in the crack distribution and the presence of broken fibers, are in Reference 58.)

The large change in $\tau$ with temperature is unlikely to be associated with thermal strains, because the matrix and fibers are expected to be very close in thermal expansion. A more likely source is a change in the shear properties of the BN coating surrounding each fiber. The change in $\tau$ implies that the critical stress for steady-state matrix cracking drops by $40 \%$.

Other experiments are also available for measuring the friction stress. In materials in which the fibers and matrix possess different thermal expansions, the fiber ends on a section of the composite cyclically protrude and retreat from the surface when the temperature is cycled. Shear lag analysis of the cyclic displacements, measured by using, e.g., $\mu$ CT, yields values of $\tau$ for individual fibers, allowing the variability in $\tau$ to be surveyed (166). Testing carried out in an oxidizing environment would reveal the changes in $\tau$ due to progressive oxidation of the interfaces.

www.annualreviews.org • Stochastic Virtual Tests

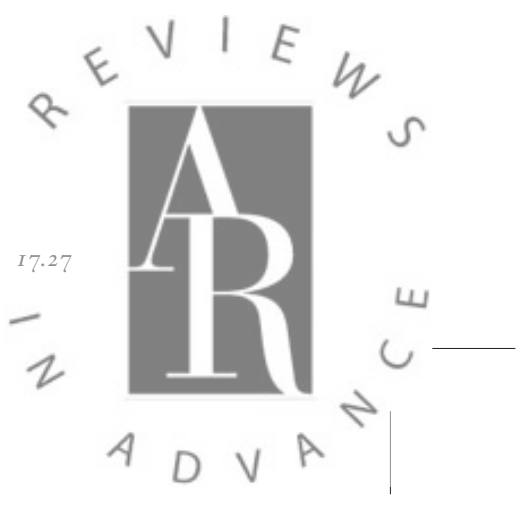


a

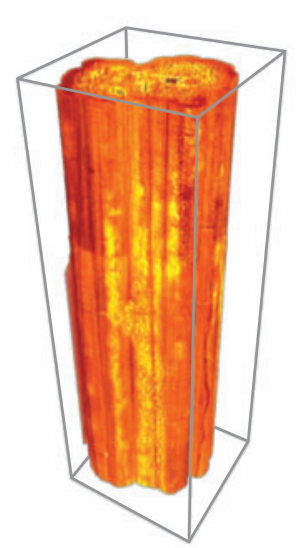

$127 \mathrm{~N}$

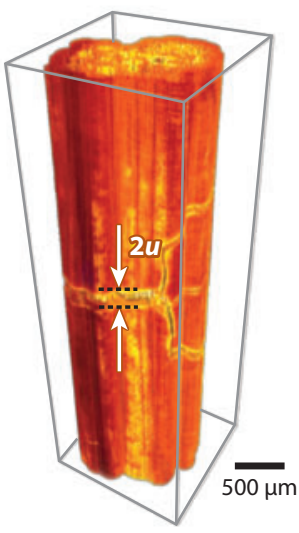

b

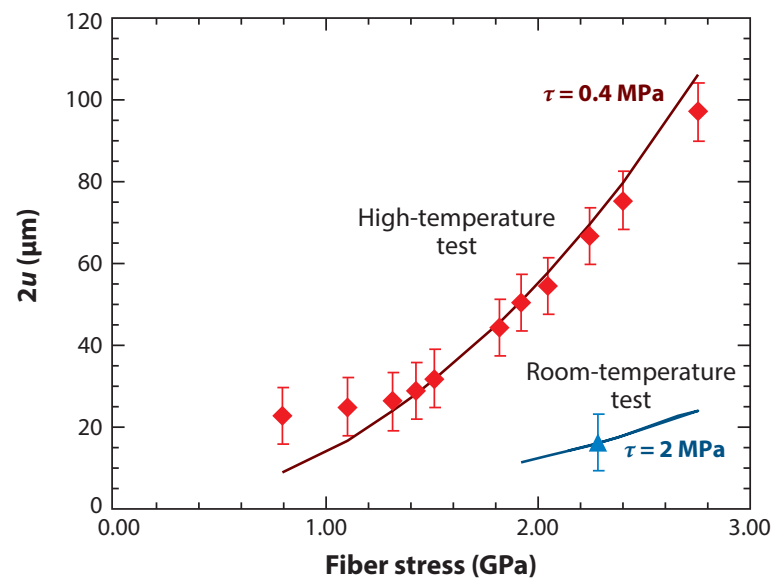

Figure 15

(a) $\mu \mathrm{CT}$ images at $1,750^{\circ} \mathrm{C}$ of a single-tow $\mathrm{SiC} / \mathrm{SiC}$ minicomposite loaded in tension. (Left) At low (10-N) load before any cracking occurs. (Right) At a load of $127 \mathrm{~N}$ after formation of a matrix crack that extends across the entire specimen while being bridged with intact fibers. (b) Crack-opening displacement data for the test in panel $a$ and a similar test at room temperature yield a fiber/matrix friction stress $\tau$ at room temperature and elevated temperature. The solid curves are fitted predictions based on shear lag theory.

\section{RAPID COMPUTATION OF MULTIPLE DISCRETE DAMAGE EVENTS}

A critical element of replicating a real experiment in a simulation is the ability to introduce new cracks during the execution of the simulation at arbitrary locations and with arbitrary orientations, as determined by the current local stress or strain fields. However, this objective has been difficult to achieve; the discontinuous nature of localized damage cannot be directly modeled by the standard FEM. The first major breakthrough came in the theory of the partition of unity $(\mathrm{PoU})$ proposed by Babuška \& Melenk $(167,168)$, from which the generalized FEM (G-FEM) was developed. The PoU theory allows for a priori knowledge about the solutions of prototypical boundary value problems (e.g., notches/corners, voids, and cracks) that arise in a simulation to be incorporated into the numerical methods used through an enriched numerical scheme (169-172). During the same time period, Belytschko and colleagues (19, 173-177) independently developed the extended FEM (XFEM), which permits arbitrary discontinuities (static or propagating) to arise in a simulation without the need for remeshing. Xiao \& Karihaloo (178) and Belytschko et al. (179) recently reviewed developments in the formulation and successful applications of the X-FEM and the G-FEM.

The crack tip enrichment scheme in the X-FEM has the practical drawback of being inherently nonlocal: When one element cracks, neighboring elements are involved in the correction to the local field. Although X-FEM can deal well with the near-tip stress-strain field of an individual crack, nonlocality complicates the problem of accounting for crack interactions such as coalescence and bifurcation. Correct and efficient treatment of coalescence and bifurcation is critical for simulating the complex, multicrack damage states found in composite materials.

In composite failure analysis, another class of methods is therefore more widely used. These

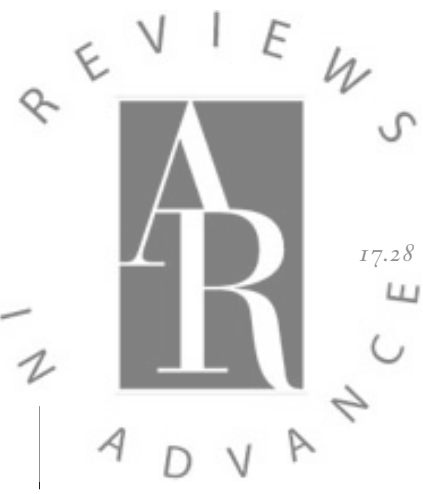
methods are derived from the seminal work of Hansbo \& Hansbo (180) and Belytschko et al. (181), who introduced double nodes to describe an intraelement discontinuity in a numerical augmentation that is local: Only the element directly affected by a discontinuity (crack) need be modified. Under the name phantom node method (PNM), its first applications were to dynamic

Cox et al. 
cracking and shear banding in thin plates (182), after which it was used for composite materials (183-185).

The augmented FEM (A-FEM), which was developed by Yang and colleagues (186-188) and is similar to the conceptual element in the PNM but without the use of PoU methods, has also been successfully used to study multiple arbitrary cracking problems in laminated composites. The PNM and A-FEM do not represent the crack tip as singular but assume that stress remains finite due to the action of material nonlinearity. The methods can be readily combined with cohesive zone models of nonlinear fracture to yield unified, realistic descriptions of crack initiation in pristine materials and the subsequent development of a propagating crack $(162,189-191)$. The methods have succeeded in a number of in-depth material studies $(184,188,192)$. The PNM and A-FEM use only standard shape functions, and all degrees of freedom are physical (i.e., correspond to real material displacements), making them compatible with any commercial FEM code. Recently, Van der Meer (192) comprehensively reviewed the PNM, the A-FEM, and the regularized FEM, which was developed by Iarve and colleagues (193-195).

A recent reformulation of the A-FEM improved its ability to treat general crack configurations and its speed $(25,196)$. In the new formulation, there is no need for external virtual nodes to account for the discontinuous displacement fields; internal nodes are used with degrees of freedom that can be condensed out from the elemental equilibrium equation. The use of external virtual nodes in the original formulation, and in other formulations of similar elements, complicates the treatment of multiple interacting cracks within a single element. By using internal nodes only, the new formulation makes accounting for multiple interacting cracks very easy and avoids computationally costly tracking algorithms.

Key attributes of the A-FEM include 3D elements that allow cracks to be introduced on any plane within an element, with cohesive tractions acting on the fracture surfaces that follow a prescribed nonlinear fracture law; permission of multiple cracks within a single element; and correct computation of the local stresses and cohesive traction distributions around bifurcation or coalescence events (Figure 16). The A-FEM correctly tracks the discontinuities in displacement fields that are introduced by cracks within elements, as well as the discontinuity in the cohesive tractions acting along one crack when it is impinged upon by a second crack (25).

In addition to allowing for the correct description of key multiple crack configurations, the reformulated A-FEM incorporates a new iteration algorithm for searching for convergence in nonlinear problems, in which the global stiffness matrix is rewritten in a piecewise linear form. This development allows local convergence to be achieved in one or at most a few steps. The combination of the new iteration algorithm, the compact definition of a multi-ply cracked element, and the use of advanced quadrature algorithms that permit an element to be somewhat larger than the length of the fracture process zone leads to high computational speed $(25,196)$. For a single crack in a bending beam or a delamination crack, a solution to failure, including accurate analysis of snapback in the case of the bending beam, takes several seconds on a standard workstation of 2012 vintage. Programmed on a graphics card, computation of problems with 10 or even 100 cracks should be possible in a similar time frame.

Computational speed is essential in a virtual-test strategy that seeks to address stochastic variability. As discussed below, a challenging but feasible near-term target time of $1 \mathrm{~s}$ per simulation would meet the demand of typical case studies.

\section{VALIDATION STEPS}

The most important validation for a design engineer refers to the ability of a virtual test to correctly predict the outcome of a real test. For simulations that refer to stochastic details of the

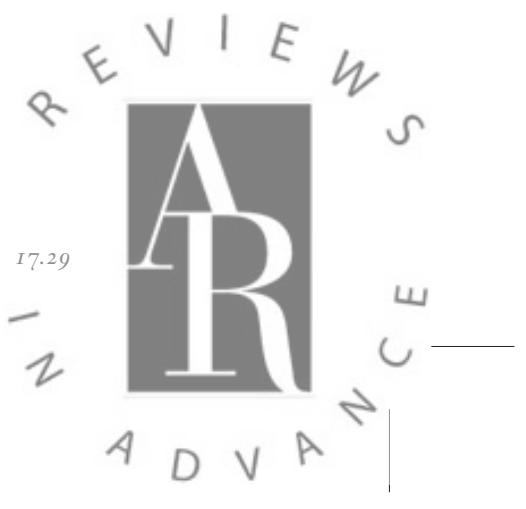




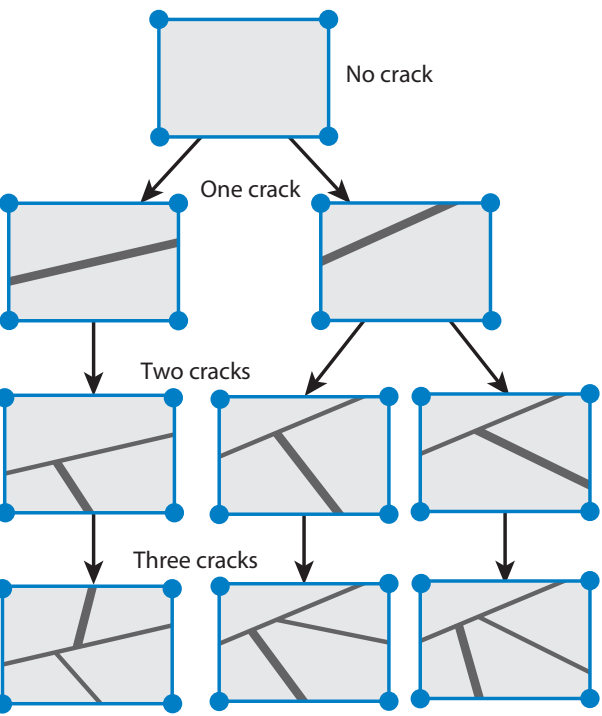

Figure 16

In the reformulated augmented finite-element method (A-FEM), a single element can split multiple times, accommodating crack bifurcation or coalescence events. The cracks can follow preexisting material boundaries or other paths determined by local stress conditions. Regardless of the number of cracks, the global degrees of freedom and the elemental equilibrium equation maintain the same structure as that of a regular element. (The examples shown in this figure do not exhaust all possible combinations of intercepts of cracks with the element boundaries.)

microstructure, an important intermediate validation step addresses the question of whether the microstructure was correctly replicated in an ensemble of generated virtual specimens.

\subsection{Validation of Virtual Specimens}

Once the decision has been made that a certain set of characteristics are to be used to represent a microstructure, statistics for those characteristics can be compiled by analyzing data from images. The statistics can be summarized in a data set, as in Equation 4. One validation of a virtualspecimen generator is to check that the ensemble of generated specimens does indeed replicate this target data set. Proof of correct replication for the Markov chain algorithm used for the unit cell scale can be found in References 74, 95, and 107.

Validation against measurements that are independent of those used to create the target statistics is also desirable. The topography of the surface of a textile determines, among other things, the thickness of the superficial layer of matrix that will be deposited to form an aerodynamically smooth surface. The thickness of the layer partly determines the critical stress for cracking (197), which is therefore influenced by the geometrical variations in the textile surface. Figure $\mathbf{8}$ shows the excellent agreement of the surface topography of virtual specimens generated by the Markov chain algorithm with the surface topography of real specimens, as measured by DIC. The DIC data are a different experimental source than the $\mu \mathrm{CT}$ data used to calibrate the virtual-specimen generator.

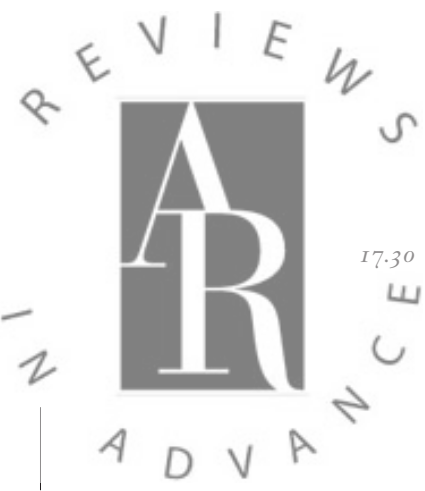

\subsection{Failure Predictions}

The case study illustrated in Figure 14 offers one successful instance of validation of a simulation. Cohesive laws and elasticity calibrated by a distinct set of tests led to quantitative prediction of

Cox et al. 
the location of initial cracking, a regime of stable crack growth, and the location of new crack initiation at higher loads, along with most of the nonlinear load-displacement curve for a test of a subcomponent with a much more complex geometry than that of either calibrating test.

The objective of validating predictions of failure at the tow scale in a $3 \mathrm{D}$ textile composite introduces some complexity not present in the case of Figure 14. The subcomponent of Figure 14, as it is laminated, failed in an essentially deterministic manner via a relatively simple system of delamination cracks that mainly followed nondeviating paths between laminae. The accomplishment of Figure 14 is significant, but validating predictions of microcracking at the tow scale in a heterogeneous textile with a stochastic microstructure (e.g., Figures $\mathbf{1 0}$ and $\mathbf{1 7 a}$ ) is substantially more difficult.

\subsection{Simulations of Complex Damage Evolution}

The most useful validating data for microcracking predictions are the locations, shapes, and sizes of all the cracks that arise throughout the volume of a tested specimen. Confirmation must be sought that a simulation technique such as the A-FEM method, when used to calculate failure in a virtual specimen constructed by using the methods of Section 3 and calibrated as described in Section 5 , replicates the multiple mechanisms of failure seen in experiments.

One such validation exercise addressed a carbon/ $\mathrm{SiC}$ angle interlock similar to that in Figure 3, but with additional monolithic $\mathrm{SiC}$ matrix infiltrated between and around the coated fiber tows to form a composite sheet with smooth upper and lower surfaces. The virtual specimen was generated with stochastic tow geometry through Monte Carlo methods (95), with the statistics of variance matching those measured by $\mu \mathrm{CT}$ (9). The geometrical model resulting from application of the Markov chain algorithm of Section 3 was converted into a mesh of solid computational elements suited to the A-FEM formulation. The isotropic elasticity of the added matrix material and the distinct, transversely isotropic elasticity of the fiber/matrix material in tows were calibrated by analyzing tests on two specimen types, i.e., the composite of Figure 3 (infiltrated tows with minimal additional matrix) and the composite with additional matrix added to achieve smooth surfaces. The calibrated tow elasticity is numerically similar to the elasticity deduced for a similar carbon/SiC material, as reported in Reference 122. Nonlinear fracture laws were calibrated from the same tests by matching the predicted matrix cracking stress with the experiment.

Experiments reveal that, when the subject material is loaded in tension parallel to the warp fibers, approximately periodic patterns of microcracks develop in the added matrix that lies over the surface weft tows (Figure 17a). Both the periodicity of the pattern and the locations of the cracks relative to underlying weft tows are well predicted by virtual-test simulations using the calibrated virtual specimens. One particular characteristic of the microcracking demonstrates the potential of virtual tests for predicting details. The microcracks seen in experiments form above weft tows and propagate both into the composite and across the surface of the composite, tracking the weft tow. However, the propagation across the surface is limited: When the microcrack approaches the region where the weft tow dips beneath a warp tow, propagation is arrested. Thus, the microcracks remain of finite length (Figure 17a). The simulations replicate this behavior very well. The images of panels $b$ and $c$ of Figure $\mathbf{1 7}$ focus on a single microcrack in the matrix layer. The microcrack follows a weft tow and then arrests as it approaches the location where the weft tow is submerged under a warp tow.

The phenomenon of crack arrest is correlated with lowering of stress in the superficial matrix by straightening of the warp tow. [Berbon et al. (121) reported earlier evidence for tow straightening in this class of composites.] Both stress heterogeneity and the critical stress for microcracking depend on geometrical details such as the thickness of the superficial matrix layer and the orientations of

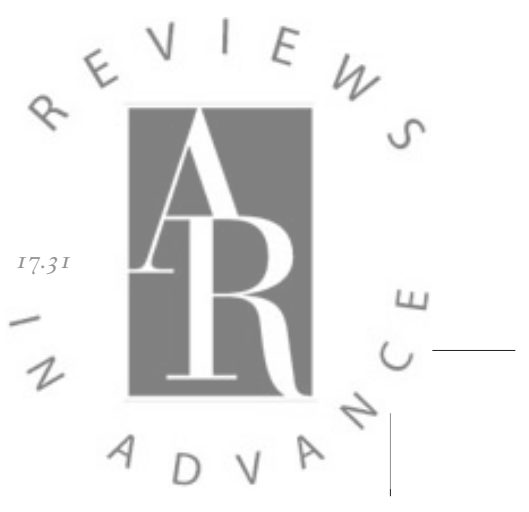


a
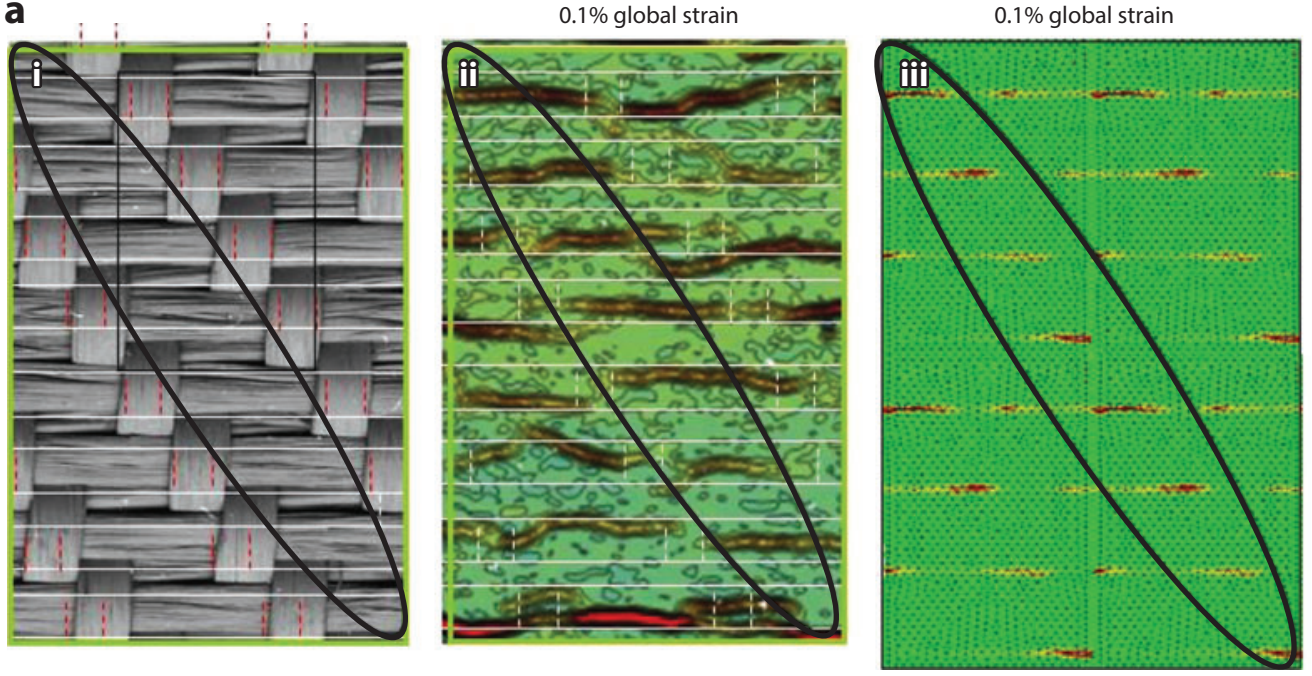

$E$, maximum principal (average: $75 \%$ )
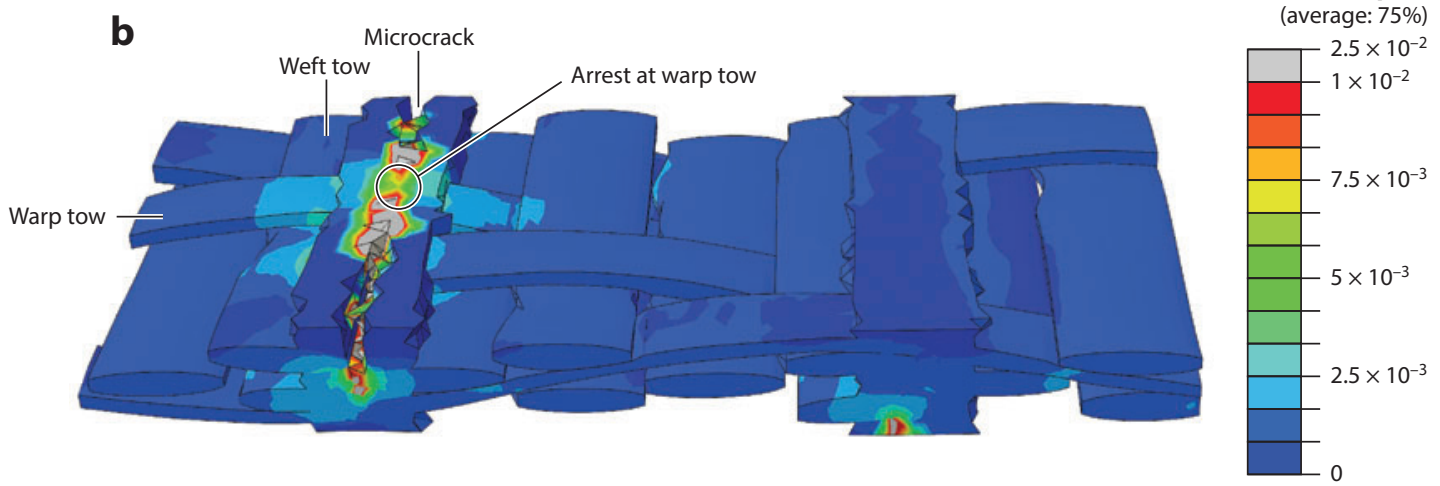

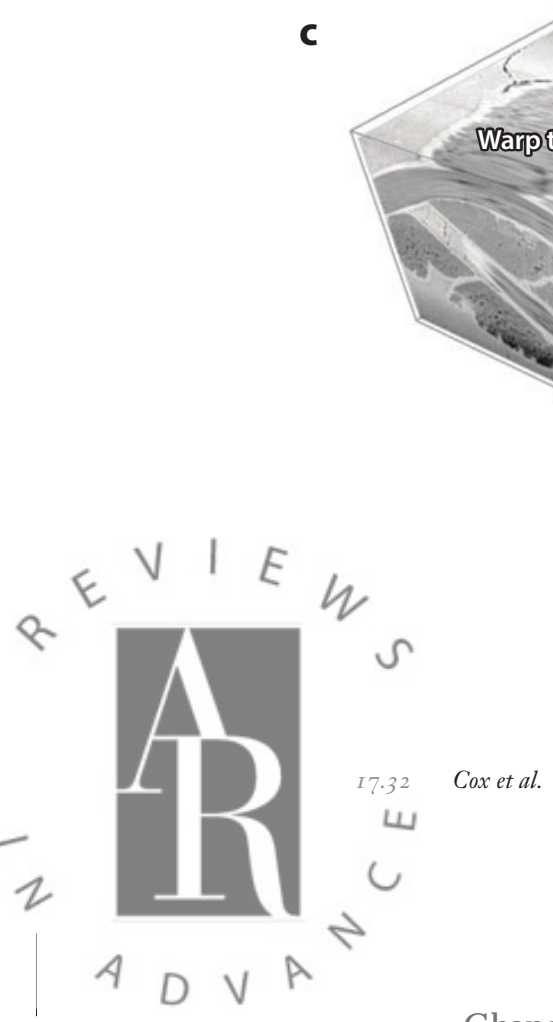

C hanges may still occur before final publication online and in print 
tows. The critical stress for microcracking is therefore affected by stochastic variance in the tow architecture. By simulating the evolution of discrete crack systems with high fidelity, the virtual tests can directly relate variance in microstructure to variance in failure due to microcracking (a potentially fatal event in hot structures).

Current research seeks to define quantitative metrics by which success in predictions can be gauged, such as crack length and the density of cracks per unit volume. Validation is being addressed for varying temperatures, environments, and load conditions, a challenging exercise that is likely to continue for many years. Because of the stochastic nature of the textile microstructure, validation will be sought for predictions of the distribution of properties for an ensemble of virtual and real specimens, rather than just for predictions of mean properties.

For high temperature, $\mu \mathrm{CT}$ measurements are by far the richest source of required data; in fact, the advent of high-temperature $\mu \mathrm{CT}$ has recast the entire concept of what constitutes a rational virtual test. A key current objective is to develop standardized coupon tests that are conducted at high temperature in X-ray tomography beamlines and that can be used reliably by design engineers in the field.

\section{THE PREDICTION OF PROBABILITY DISTRIBUTIONS}

Among the potential uses of a validated virtual test is the prediction of distributions of strength and life and the relation of variance in properties to variance in the material. In the example shown in Figure 17, variations in the thickness of the matrix layer at the location of a microcrack or in the orientation of the tows that lie under the crack change the local stress state and therefore the crack driving force. The microcrack evolution is thus a stochastic process, as implied by the irregularity of the experimentally observed crack pattern. This section addresses the question of how to build predictions of statistical distributions of strength, life, and other performance metrics that depend on such stochastic phenomena.

The two general approaches that are available exploit a virtual test as a computational engine. The first approach uses the Monte Carlo method to accumulate statistics by the repetition of simulations for different virtual specimens. The second approach uses predictions of a selection of simulations with varied parameters to establish rate equations for damage parameters, which are then used to compute the evolution of probability distributions for the damage parameters in a configuration space. Monte Carlo methods are more straightforward to implement; probabilistic models offer potentially large advantages in computational speed.

Figure 17

(a) (i) Textile preform coated with a thin layer of matrix (carbon/SiC angle interlock, similar to Figure 3). (ii) Observations of microcracks by digital image correlation in the composite formed by full infiltration of the SiC matrix into the preform in panel $a$. (iii) The predicted crack pattern. The three images in panel $a$ are spatially registered so that textile features appear in the same place in each image. Key features of the crack pattern-including the approximate periodicity of the microcracks, which form above nearsurface weft tows, and the alignment of microcracks in diagonal bands (one of which is outlined by the ellipse) —are well replicated by the virtual tests. (Image $i i i$ actually shows a material in which the weft tow spacing was larger than in image $i$ or image $i i$, but this material is otherwise of identical architecture; image $i i i$ was rescaled to facilitate comparisons. Importantly, the relation of crack position to weft tow position is the same in all three images in panel $a$.) (b) Detailed rendering of a simulation shows one microcrack above a weft tow correctly predicted to arrest upon approaching the underlying warp tow. The matrix containing the crack is pres $\$$ nt in this image, but the matrix elsewhere was stripped away in rendering the image to reveal the underlying tow architecture. (c) 3D visualization of $\mu \mathrm{CT}$ data provides further detail of the observation in panel $a$ that matrix cracks that have formedabove a weft tow segment arrest when they approach the region where a warp tow crosses over the weft tow. All such warp tow segments are indicated by the vertical dashed lines in panel $a$; these segments correlate consistently with crack arrest.

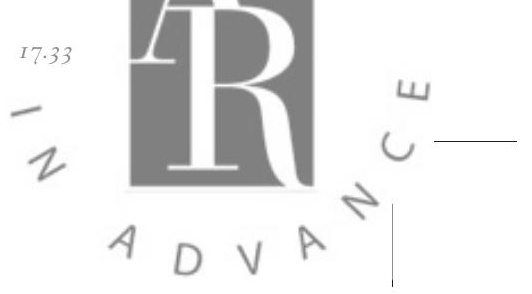


Both Monte Carlo and probabilistic approaches have been used for several decades to predict fatigue life for metal alloys (198-204). For alloys, one key problem is predicting the varying growth rate of a crack as it interacts with the granular microstructure of the alloy, especially when the crack is still small relative to the grain size $(205,206)$. Because the grains are random in size, shape, and crystalline orientation, the crack growth is a random process.

In some textile ceramic composites, crack growth during cyclic mechanical loading also occurs. In carbon/SiC composites, for example, matrix cracks progress across a tow as the carbon fibers are degraded by mechanical attrition, which weakens their bridging effect. This process occurs at a much faster rate in vacuum at elevated temperature $\left(850^{\circ} \mathrm{C}\right)$ than at room temperature (114). But other rate processes, including oxidation, flow of glassy phases, and creep, also contribute to life determination in ceramic composites, especially at high temperatures. The rate at which these mechanisms act depends on the random microstructure. Loss of surface material due to oxidation, for example, leads to failure upon loss of the matrix overlayer, which has random thickness. Ingress of oxygen that attacks interior fibers is accelerated by microcracking, which, like creep and fatigue crack growth rates, is influenced by variance in the geometry of the textile reinforcement.

\subsection{Monte Carlo Methods}

The Monte Carlo method applied to ensembles of stochastic virtual specimens provides the closest analog to a real test matrix. A statistically significant number of virtual specimens are subjected to test conditions that are the same as those used in the real tests, and engineering predictions are deduced from the mean and scatter in the outcomes $(207,208)$. Each virtual specimen in the simulated ensemble is one instance of a random microstructure that was constructed by feeding pseudorandom numbers into calibrated distribution functions (a Monte Carlo procedure). Variance in the microstructure in the ensemble of virtual specimens leads to variance in predictions, thus matching at least part of the variance observed in real tests. With trivial modification, the load in the virtual test can also be made random, e.g., to simulate random overloads in a duty cycle, leading to prediction of a further possible contribution to randomness in real tests.

Once a stochastic virtual-specimen generator has been developed and constitutive laws have been calibrated, executing a Monte Carlo analysis is straightforward. Simulations such as those described in Section 6 are executed for each member of the ensemble of virtual specimens, and predicted metrics (e.g., strength, strain to failure) are analyzed through the same statistical methods used to analyze real tests.

An early illustration of Monte Carlo methods based on simulations appeared in References 80 and 208 and may indeed be the first example of a virtual test defined by the six steps listed in Section 1 (although the phrase virtual test was not in use in that era). (a) The characteristics of grains in alloy specimens were measured. (b) The statistics of the grain shapes, especially the average area and the aspect ratio, were determined. $(c)$ An ensemble of virtual specimens was generated by using Voronoi polygons to represent grains, and the polygons' dimensions were calibrated against the measured grain sizes and shapes. $(d)$ Crack growth laws were associated with the material in the virtual specimens, which were based on and calibrated by detailed experimental observations of the interaction of cracks with grain boundaries in certain aluminum alloys (209). (e) Crack growth simulations were executed by using weight functions to compute variations in stress intensity factor around an evolving crack front made irregular in shape by its interaction with the grain structure. The statistics of crack growth and the influence of microstructure on life distributions were deduced from Monte Carlo ensembles of simulations. $(f)$ Partial validation was achieved by comparing predicted distributions and measured distributions of crack growth rates.

Cox et al. 
Table 1 Influence matrix for polymer composite laminates in tension

\begin{tabular}{l|c|c|c|c}
\hline \multirow{2}{*}{$\begin{array}{l}\text { Cohesive } \\
\text { parameter }\end{array}$} & \multirow{2}{*}{$\begin{array}{c}\text { Estimated uncertainty } \\
\text { in cohesive parameter }\end{array}$} & $\begin{array}{l}\text { Ultimate } \\
\text { strength }\end{array}$ & $\begin{array}{c}\text { Global stiffness in } \\
\text { nonlinear regime }\end{array}$ & $\begin{array}{c}\text { Splitting-crack } \\
\text { growth rate }\end{array}$ \\
\hline$G_{\text {Ic }}$ & $10 \%$ & 0.1 & Small & 0 \\
\hline$G_{I \mathrm{Ic}}$ & $10 \%$ & $0.25^{\mathrm{a}}$ & Small & $-1^{\mathrm{a}}$ \\
\hline$\hat{s}_{\mathrm{n}}$ & $15 \%$ & 0.3 & 0.1 & 0 \\
\hline$\hat{\tau}_{s}\left(=\hat{\tau}_{t}\right)$ & $10 \%$ & 0.3 & 0.2 & -1 \\
\hline
\end{tabular}

The estimates of uncertainty in cohesive law parameters following calibration are listed, followed by the influence matrix components relating the four parameters and three key metrics of composite failure. $\phi$ denotes an engineering property, and $\theta$ denotes a cohesive parameter.

${ }^{a}$ Very nonlinear dependence.

8.1.1. Influence matrices. A statistical outcome of particular interest in material optimization is the influence matrix, defined here as the matrix of partial derivatives of a predicted engineering property with respect to any geometrical or material characteristic used to define a virtual specimen. An example of such an influence matrix appears in a recent study of a polymer composite laminate failing under monotonic tension (24). The laminate fails via a system of mixed-mode cracks, each of which is governed by cohesive laws that were built by combining separate mode I and mode II laws. Both the mode I and mode II laws are defined by a strength and a fracture energy, yielding the four parameters in column 1 of Table 1 . The cohesive parameters were independently calibrated; the uncertainty in the calibration (which reflects material scatter in the calibration tests) is listed in column 2 of Table 1.

The remaining entries in Table 1 report the elements of the influence matrix, which have the normalized form $\partial\left(\phi / \phi_{0}\right) / \partial\left(\theta / \theta_{0}\right)$, with $\phi$ an engineering property and $\phi_{0}$ its mean value, and $\theta$ a fracture law parameter and $\theta_{0}$ its mean value. The influence derivatives were determined by executing simulations in which the cohesive parameters were varied.

As well as revealing which fracture parameters most strongly influence which properties (some influences are not as intuitively obvious as others), the influence matrix also allows for prediction of the scatter expected in properties; i.e., the influence matrix can be used to determine a covariance matrix. For example, the scatter in ultimate strength due to the measured material variance in mode I fracture toughness, $G_{\mathrm{Ic}}$, is predicted to be $0.1 \times 10 \%=1 \%$; i.e., $G_{\mathrm{Ic}}$ has a modest effect. In contrast, the scatter in the ultimate strength due to variance in either $G_{\text {IIc }}$, the tensile cohesive strength $\hat{s}$, or the shear cohesive strength $\hat{\tau}$ is larger because the corresponding influence factors are larger. The combined effects of the variance in all fracture parameters imply scatter of approximately $10 \%$ in the ultimate strength, which agrees encouragingly well with the measured scatter (24).

8.1.2. Computational demands. The computational expense of Monte Carlo analysis can be high. The uncertainty $\sigma$ in a predicted mean property, such as the expected strength in a distribution of strengths, falls as the number of computed cases $N$ increases according to $\sigma \approx N^{-1 / 2}$. For example, if strength is predicted to have a scatter of $10 \%$, then determining the mean strength to $1 \%$ accuracy requires 100 simulations.

When a virtual test is used in material design, a design optimization study may require computing the relative merits of $10^{2}-10^{4}$ different combinations of material or architectural parameters. To establish reasonably accurate trends in mean strength, 100 simulations may be required for

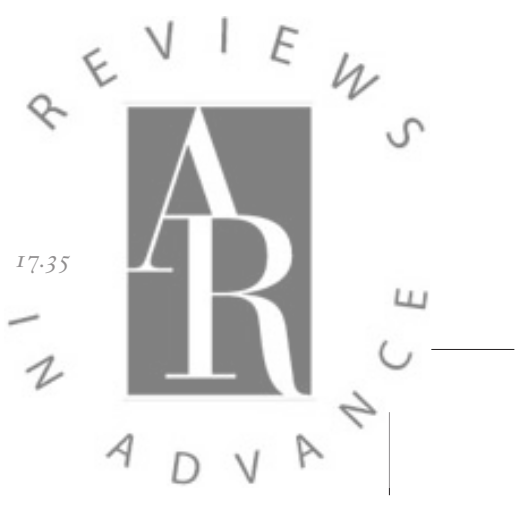


each combination, and thus $10^{4}-10^{6}$ virtual tests must be executed. For the computational time to remain within 1 week $\left(10^{6} \mathrm{~s}\right)$ for a relatively wide search, a single virtual test should run in $1 \mathrm{~s}$, to order of magnitude. Although computational speeds continue to improve as better algorithms are developed (see Section 6), current execution times for failure resulting from multiple cracks remain at least one to two orders of magnitude higher than the 1-s target, even for relatively simple crack configurations (25).

When a virtual test is used to certify a material, it must predict not just mean properties but also extreme properties, i.e., the probability of occurrence of a rare but fatal outcome. To predict a failure with a $10^{-7}$ or even a $10^{-5}$ probability from an ensemble of $10^{4}-10^{6}$ virtual tests necessitates extrapolating the computed statistics of failure from the domain in which data are dense into a domain in which data are likely to be sparse (the extreme tail of the failure distribution). This extrapolation can be very inaccurate. Of particular concern is the possibility that a local configuration of the microstructure might strongly influence lifetime but not be represented in the particular Monte Carlo ensemble that was generated. Examples of extreme predicted fatigue crack growth rates associated with unusual configurations of adjacent large grains were demonstrated in Monte Carlo simulations of small fatigue crack growth in alloys, with the alarming characteristic that their probability could not be predicted from the statistics of cases near the mode (208). If such possibilities need to be addressed, the required number of Monte Carlo simulations may be unreachable.

\subsection{Probabilistic Theories}

Probabilistic models offer computational efficiency and the possibility of accurate predictions of rare events. In a probabilistic theory, instead of tracking damage evolution through a particular microstructure, one tracks the evolution of a probability distribution $P(X)$ for a damage variable $X$ through time or elapsed fatigue cycles. Because $P(X)$ can be represented numerically with arbitrary precision over all values of $X$, accuracy in predicting the tails of the distribution is at least possible. However, accuracy is not assured: It also demands that the probabilistic theory incorporate a faithful representation of the details of the influence of the stochastic microstructure on the evolution of $P(X)$.

A slender literature presents two approaches to incorporating the influence of microstructure in a probabilistic formulation. We now discuss both approaches in turn.

8.2.1. Phenomenological probabilistic models. The first and simplest approach uses diffusion equations or discrete chain models to describe the evolution of a damage variable such as crack length, coupled to a simplified representation of the influence of some other factor, which could be a microstructural factor. An equation describing the evolution of a probability distribution for a continuous variable such as crack length has a form similar to the Fokker-Planck equation (210, 211). If the evolution is a Markovian process, the related Chapman-Kolmogorov equation can be employed; this equation reduces to a Markov chain model if the random variable is discrete (212). In early applications to fatigue crack growth, Bogdanoff and colleagues $(200,201,213)$ built Markov chain models (B-models) of the evolution of $P(X)$, with $P$ denoting probability and $X$ the crack length. The probability of $X$ taking a value $X_{2}$ at time $t_{2}$ given its value $X_{1}$ at time $t_{1}$ was considered to be stochastic because of the effects of variability in manufacturing conditions or load (200, 201,213). These conditional probabilities form a PTM when $X_{1}$ and $X_{2}$ are discretized, and the evolution of the probability distribution for the damage variable becomes a problem in matrix

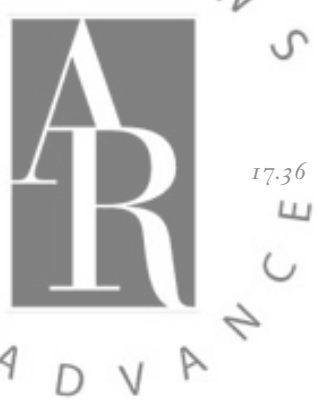


algebra. Thus, the B-models are amenable to analytical solution, even when the evolution process is not uniform in time (i.e., is nonstationary) (213).

A modification of this method [a semi-Markov process $(214,215)$ ] introduces a subsidiary variable $Y$ representing variability in microstructure such that the probability of $X$ taking a value $X_{2}$ at time $t_{2}$ given its value $X_{1}$ at time $t_{1}$ depends not only on $X_{1}$ but also on $Y\left(t_{1}\right)(216,217)$. Thus, the PTM has elements that are functions of $Y$. The explicit presence of variable $Y$ allows the probability of local damage evolution to depend on the local microstructure present in the neighborhood of a crack at time $t$, which is represented by $Y$. An early example of this class of models was solved by Monte Carlo methods, with $Y$ sampled from a distribution representing the statistics of the microstructure (217). But whether solved analytically or by Monte Carlo methods, phenomenological models differ fundamentally from Monte Carlo studies of virtual specimens in that they do not follow the evolution of the damage itself in a single specimen but rather follow the evolution of the probability distribution of a damage variable. Such models can be solved rapidly and precisely to predict the entire distribution function for failure, including low-probability tails.

However, the phenomenological models are limited in the degree of fidelity they can achieve in predicting the effects of microstructural variation within a single class of materials. Such models typically use at most a single parameter such as $Y(t)$ to represent microstructure, which is calibrated by using data for materials that are identical to those for which predictions are to be made, with the same failure mechanisms and the same microstructural statistics. These models cannot easily be used to make predictions for materials with different microstructural statistics or with different loading conditions. Improved fidelity requires the incorporation of further details of how microstructure controls the evolution of local damage events.

8.2.2. Probabilistic models with more details of microstructural influences. One probabilistic formulation that seeks higher fidelity was presented by Pardee and colleagues $(218,219)$ to address small fatigue crack growth in alloys. The theory invokes laws determined by experiments to relate the rate of growth of a crack to the values of certain local microstructural characteristics (such as grain size). Because alloy microstructure is stochastic, the microstructural characteristics are defined by probability distributions. The theory tracks the evolution of probability density functions for crack length in a large population of cracks, such as might exist in a large ensemble of specimens. These researchers explicitly account for multiple mechanisms of the interaction of a crack with the microstructure, including crack arrest at grain boundaries and the influence of grain size on slip band development. The existence of grain boundary arrest implies that probability density functions depend on different microstructural parameters at different stages of damage evolution. Accordingly, in the theory, damage evolution occurs in distinct stages of alternating crack growth and arrest, which are modeled by including a distinct variable domain in probability space for each stage, with the variables for each domain chosen according to whether the crack is growing or arrested (219). Although such a formulation presents relatively complex numerical and programming challenges, it retains the efficiency of a probabilistic method while offering much greater fidelity to the physics underlying damage evolution than do common phenomenological models.

The virtual-test paradigm can couple to a Pardee-like formulation in several ways. First, distributions of microstructural parameters that appear in damage growth laws can be computed by analyzing virtual specimens. Second, stress analysis of virtual specimens yields the distribution of the local stress in the environment of a crack or another local damage event. Third, the rate of crack propagation or other damage evolution (e.g., oxidation damage) can be related to microstructural parameters by executing virtual tests for a limited number of virtual specimens; in such tests, details of the physics of damage evolution, including the effects of local stress, material

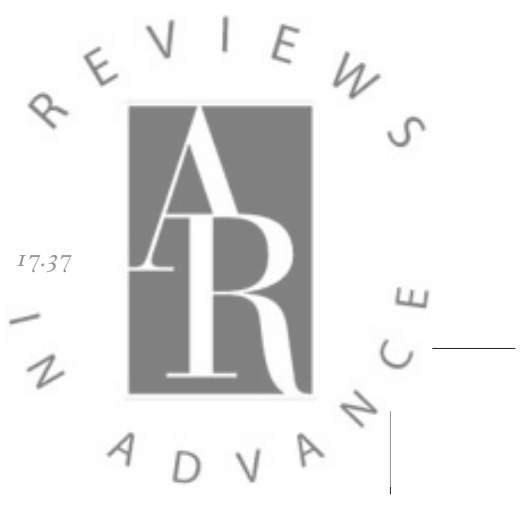


composition, and environmental parameters, are represented. Summaries of the results of such virtual tests inform the laws of growth needed in the Pardee formulation.

With growth laws and statistical distributions thus calibrated at least partly by using virtual tests, a probabilistic model can be solved to generate relatively rapid predictions of the full distribution function for engineering properties, such as time to failure. Thus, virtual-test methods can be exploited to probe the statistics of rare failure events within a computationally efficient tool.

\section{THE FIBER SCALE}

The methods illustrated above for executing virtual tests at the tow scale are based on wellestablished engineering principles: The concepts of the nonlinear fracture mechanics required to describe cracking events at the tow scale have been in the literature for decades, and calibration and validation data for material properties and cohesive fracture laws can be supplied by familiar standardized tests. In contrast, extending the virtual-test system to cover events first at the spatial scale of a single fiber, and then down to the atomic scale, raises fundamental questions that remain unanswered: What contribution to a practicable virtual-test system can be expected from studies at such scales, what experiments can yield sufficient information, and how can theory represent the complexity of behavior at the fiber and atomic scales?

\subsection{Fiber-Scale Virtual Specimens}

For the first time, data on the position of every individual fiber in a tow or ply are emerging from high-resolution $\mu \mathrm{CT}$ images (12). In contrast to traditional analyses based on serial sections, the $3 \mathrm{D}$ data yield the complete, continuous locus of each fiber over significant lengths of the bundle. Such data are revealing fascinating complexity in the fiber arrangements (220). Even in fiber bundles that appear well aligned to the eye, some fibers meander within the bundle, with important implications for material processing and failure. For example, bundles with greater degrees of meandering are expected to be compacted to lower volume fractions but exhibit enhanced fracture resistance under transverse loads, because meandering fibers bridge splitting cracks (117).

Establishing metrics for fiber meandering requires concepts from topology, especially graph theory, that are infrequently used in material science. In the example of Figure 18a, most fibers in the bundle shown are parallel, but one fiber meanders. The nearest neighbors of any fiber on a section perpendicular to the nominal fiber direction can be determined by the Voronoi construction (Figure 18b) (221). For the meandering fiber, the nearest-neighbor set on two section planes is different. When the neighbor map is computed for all fibers on all section planes in a data set, the degree to which a fiber meanders can be characterized by the number of distinct neighbor sets that the fiber possesses per unit length measured along the bundle (neighborhood gradient), denoted $\dot{X}$. The reciprocal of $\dot{X}$ is the average distance between points along a fiber at which the neighbor map changes, denoted $\lambda_{X}$. Distributions of $\lambda_{X}$ show marked differences for fiber bundles from different sources: For the fiber bundle in the minicomposite specimen of Figure 14, the median of $\lambda_{X}$ is approximately 55 fiber radii, and for the carbon fibers in a typical carbon/epoxy tape laminate, the median of $\lambda_{X}$ is approximately 13 fiber radii (220).

Fibers exhibiting high rates of change of neighbor map $\dot{X}$ tend to group in different ways in the two materials studied. Panels $c$ and $d$ of Figure 18 show a pair of section planes for each material; the material is cut normal to the fiber direction, and gray-scale coding represents the value of $\dot{X}$ for each fiber. For the single-tow $\mathrm{SiC} / \mathrm{SiC}$ composite, the few fibers of high $\dot{X}$ appear with no obvious spatial correlation either with each other or with features in the spatial distribution of fibers overall. For the carbon/epoxy tape laminate, fibers with high $\dot{X}$ tend to appear in ribbon-like

Cox et al. 


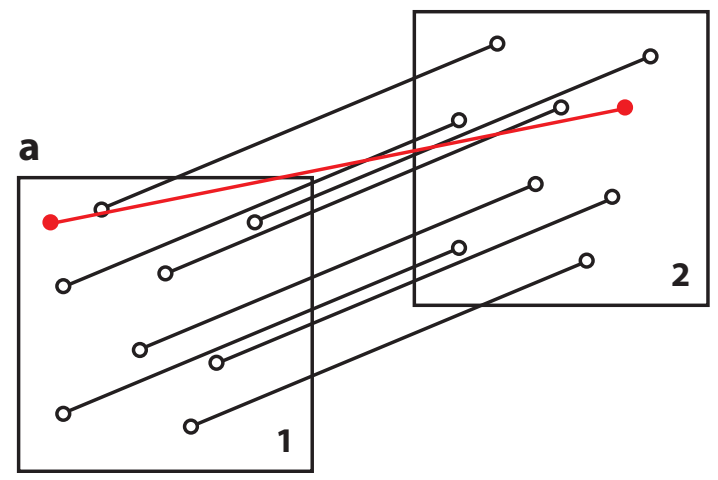

b

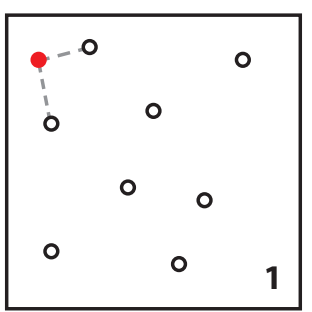

d
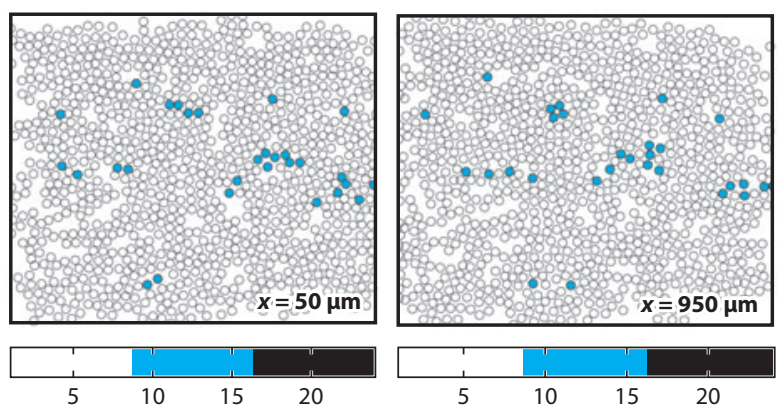

Figure 18

(a) A hypothetical fiber bundle with one meandering fiber (red). (b) Changes in the set of neighbors of the meandering fiber from section 1 to section 2. $(c, d)$ Spatial distribution of the metric $\dot{X}$ in units of (fiber radii) ${ }^{-1}$ (defined by the color code) among fibers depicted on two sections of a composite separated by $900 \mu \mathrm{m}$ along the fiber direction: $(c)$ a SiC/SiC minicomposite specimen and $(d)$ a carbon/epoxy tape laminate.

clusters, which are often near but not necessarily immediately adjacent to voids. In the laminate case, we infer that high neighbor rate change occurs in meandering fibers that lie between patches of nonmeandering fibers, with the patches shifting relative to one another as void shapes change along the fiber direction.

In analogy to Equation 4, one can devise a set of metrics that summarizes the topological characteristics of a fiber bundle and that is accompanied by more familiar Euclidean metrics (e.g., volume fraction). One should anticipate the preferred calibration set to evolve as understanding of phenomena at the fiber scale and the state of the art of measurement progress. Available data are currently limited by spatial resolution $(0.5 \mu \mathrm{m})$ and by the volume of material that can be imaged (tens of cubic millimeters). The data are rich in their content about the spatial distribution of fibers within any cross-sectional plane normal to the fiber direction but are limited in their description of variations along the fiber direction. Consideration of textile engineering (the possible presence of twist measured in turns per meter, for example) and the present data point to the possibility of variations along the fiber direction occurring over distances of $10 \mathrm{~mm}$ to $1 \mathrm{~m}$; these variations would be invisible in the data of Figure 18.

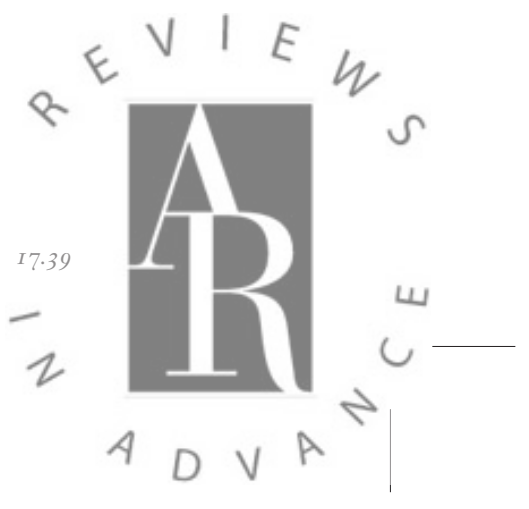




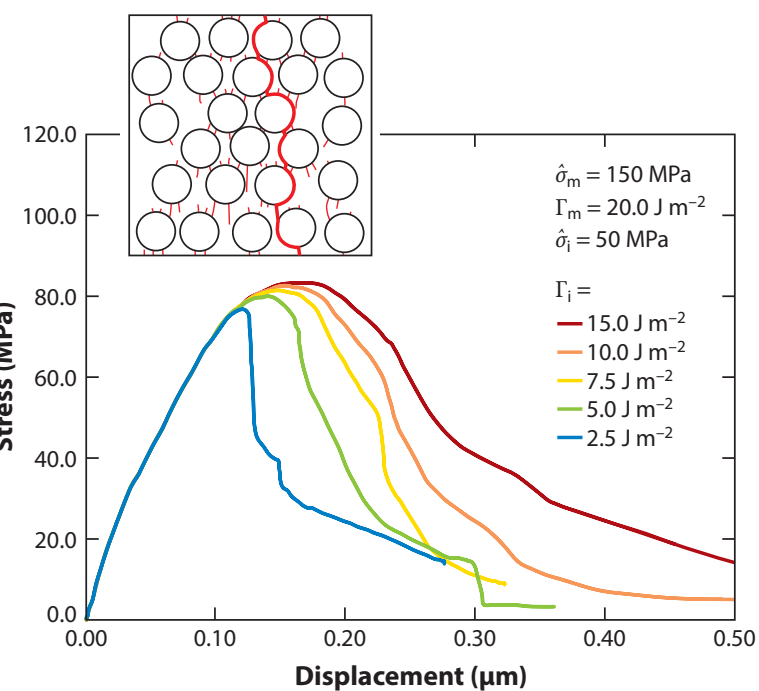

Figure 19

Simulations of transverse cracking through plies comprising random assemblies of fibers (inset) lead to relation of the global stress versus displacement law to assumed values of fiber-scale fracture properties, in this case, varying interface toughness, $\Gamma_{\mathrm{i}}$.

Fast et al. (220) suggested a metric set for use in calibrating a generator of 3D virtual specimens at the fiber scale. The metric set comprises the cumulative probability distribution for the neighborhood gradient, $\dot{X}$, along with select Euclidean metrics (local fiber volume fraction and fiber orientation). Current research seeks algorithms that will generate virtual specimens of fiber bundles possessing such target topological and Euclidean metrics.

\subsection{Fiber-Scale Simulations Predict Tow-Scale Fracture Laws}

High-fidelity simulations of damage evolution in fiber-scale virtual specimens can be used to predict cohesive fracture laws for use at the tow scale (222). Consider the case of Figure 19, which shows simulations of transverse cracks formed by the coalescence of interfacial and matrix microcracks (23). The fiber-scale simulations analyze virtual specimens of random fiber arrays, with distinct cohesive fracture laws operating at fiber/matrix interfaces and within the matrix. In this illustration, the laws were assigned a generic linear softening shape (not calibrated for any material) that is characterized by a peak stress (local material strength) and a fracture toughness. Figure 19 shows predictions of the far-field stress and displacement for various interface fracture toughness values. If the elastic displacement expected for any load is subtracted from the global displacement, each curve becomes the nonlinear cohesive fracture law for transverse ply cracking. Thus, the details of crack coalescence among random fibers in the fiber-scale simulation can be summarized by a traction-separation law for use in tow-scale simulations. When discrete crack representations of damage are used in a virtual test, the cohesive fracture law provides a physical link between scales and is the natural vehicle of a multiscale formulation.

The illustration of Figure 19 was executed by using plane strain conditions. If the fiber population were modeled in $3 \mathrm{D}$, fiber meandering would be included, and a substantial increase in the

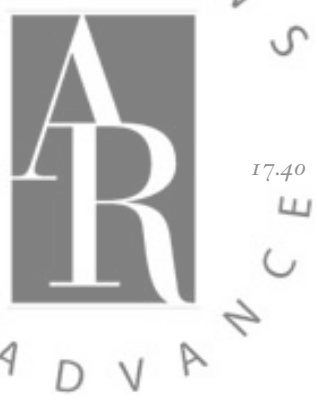

Cox et al. 
area under the predicted traction-displacement law, i.e., increased fracture toughness, would be expected.

\subsection{Complexity}

Microcracks among randomly positioned fibers, such as in the problem of Figure 19, tend to initiate first in the matrix between pairs of fibers that have the smallest separation, $s_{0}$ (e.g., see a study for brittle matrix composites in References 63 and 223). In a brittle composite with weak interfaces, the site of first initiation tends to be correlated with the path of subsequent cracking. Consider now the consequences of introducing a small perturbation to the positions of all fibers, resulting in the separation of the pair that had separation $s_{0}$ increasing to $s_{0}+\delta s$. Assume that the overall distribution of separations remains unchanged. The probability that the pair whose separation was $s_{0}$ remains the pair with the smallest separation is easily computed:

$$
p=\prod_{k} \frac{1-C P D\left(s_{0}\right)-f\left(s_{0}\right) \delta s}{1-\operatorname{CPD}\left(s_{0}\right)} .
$$

Here, $f$ is the probability density function for the separation $s$ of nearest-neighbor pairs of fibers; $C P D$ is the cumulative probability distribution corresponding to $f$; and the product is formed over all pairs, whose number is approximately $6 N$, with $N$ the number of fibers in the population. Numerical simulations of the random packing of hard cylinders show a distribution of separations approximated by an exponential function, $f \approx c_{0} e^{-s}$, with $c_{0}$ a constant (B.N. Cox, unpublished work). $p$ is easily calculated for different population sizes: $p$ falls to approximately 0.5 when $\delta s / R=$ $1 / N$ (Figure 20). For a bundle of $10^{3}$ fibers of radius $10 \mu \mathrm{m}, p<0.5$ when $\delta s=10 \mathrm{~nm}$. Thus, even a tiny uncertainty in fiber positions can change the location of the first crack initiation, with the possibility that the location of the eventual fatal crack will also change. The macroscopic outcome depends extremely sensitively on the initial conditions-the famous butterfly effect of chaos theory (224).

The question therefore arises of what is predictable and what is inherently unpredictable when one is simulating failure in stochastic composite materials at the fiber scale. For example, under what conditions can a predicted fracture location in a fiber-scale simulation be regarded as a true result of the material composition, and under what conditions would the outcome have been

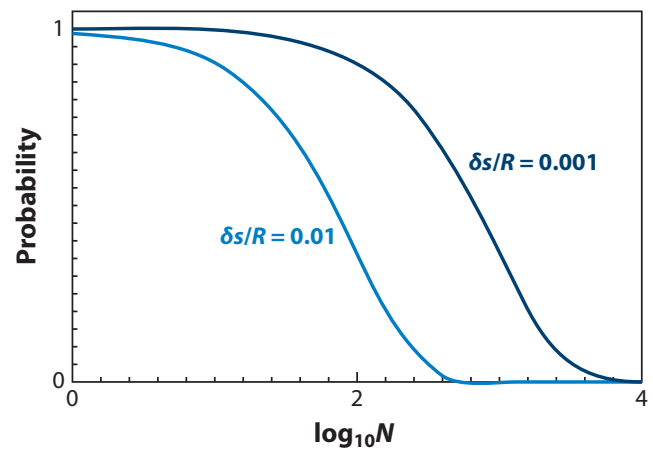

Figure 20

Probability that the pair of fibers that have the smallest separation in a population of $N$ fibers remains the pair with the smallest separation if the separation increases by $\delta s / R$, with $R$ the fiber radius.

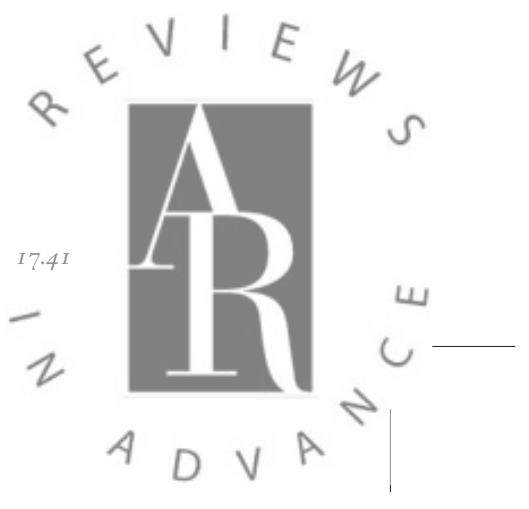


changed by a slight change in numerical methods? What characteristics of a fiber-scale simulation that can be input into a tow-scale simulation can be regarded as reliable predictions?

\section{CONCLUDING REMARKS}

This review discusses the main parts of a virtual-test system that recognizes the stochastic nature of material microstructure and that seeks to link it to variance in performance. We focus on textile ceramic composites, especially the description of discrete failure events occurring at a scale commensurate with either the width of a fiber tow or the diameter of a single fiber.

The review highlights the following advances toward a functional virtual-test system.

1. Simple standard sets of statistical metrics quantify the geometrical variances in fiber reinforcement that likely influence failure. At the tow scale, variance refers to the position and shapes of tows. At the fiber scale, both topological and Euclidean metrics are required: the former to describe the meanderings and entanglements of fibers within a tow and the latter to describe, for example, local packing densities. Analytical methods have been developed to deduce metrics from experimental images acquired by $\mu \mathrm{CT}$ for single tows or unit cell-sized specimens and by digital image analysis for subcomponent-sized specimens.

2. At the unit cell scale and the much larger scale of subcomponents, algorithms have been invented for rapidly generating ensembles of stochastic virtual specimens in which the variances of tow locations and shapes match the statistical metrics determined for real specimens. Generating virtual specimens at the fiber scale with variance matching measured topological and Euclidean metrics remains a current research topic.

3. Two new experimental systems have been developed to observe and measure damage evolution in ceramic composites at very high temperatures. A DIC system observes surface damage with optical resolution by using standard coupons at temperatures above $1,500^{\circ} \mathrm{C}$, whereas a $\mu \mathrm{CT}$ test rig determines damage with submicrometer resolution in $3 \mathrm{D}$ in miniature coupon specimens at temperatures above $1,700^{\circ} \mathrm{C}$.

4. Representative of widespread activity in developing fast simulation methods with generalized capability, a reformulation of the augmented FEM (A-FEM) has achieved high-fidelity simulation of multiple crack systems in arbitrarily heterogeneous materials. The A-FEM correctly describes the mechanics of crack bifurcation and coalescence in the presence of nonlinear fracture mechanisms acting along crack process zones. The reformulation has also achieved very high speeds relative to prior standards in multicrack simulations. Speed is vital because large ensembles of stochastic virtual specimens must be analyzed to relate failure distributions to variance in microstructure with acceptable accuracy.

5. In a display of the potential of $3 \mathrm{D} \mu \mathrm{CT}$ imaging as a probe of local material behavior, the friction stress acting at the fiber/matrix interface, which controls the question of whether matrix cracking across a fiber tow occurs in a brittle manner or in a ductile manner, has been calibrated at both room temperature and at $1,650^{\circ} \mathrm{C}$ for a representative material by using analysis of $\mu \mathrm{CT}$ fracture tests.

6. High-fidelity simulations have been used to deduce cohesive fracture law parameters from complex test data, illustrating how traditional standard test methods can complemented by tests that deal with events occurring at small scales inside a heterogeneous material. Thus calibrated, the simulations correctly predict complex cracking systems in an angle interlock weave and in laminated T-stiffener specimens. The fidelity with which the details of crack evolution can be reproduced matches that previously demonstrated for polymer tape laminate composites.

Cox et al. 
Although such successes encourage the ambition to develop virtual tests, a number of fundamental challenges remain ahead. As simulations seek to probe the influence of stochastic microstructure at the scale of the individual fiber $(\sim 1 \mu \mathrm{m})$ or at the even finer scale of random hybrid nanostructures $(\sim 1-100 \mathrm{~nm})$, the complexity of a complete material description becomes formidable. Furthermore, because the order in which failure events occur at different locations is increasingly finely balanced as the complexity of the material increases, the likelihood arises that aspects of simulations (in correspondence with nature) exhibit chaotic character. Whether the influence of increasing complexity on properties can be analyzed by simply pushing simulations of multiple discrete damage events to larger and larger problems (smaller and smaller scales) is doubtful; new methods may be required to cover multiple scales without compromising fidelity.

To complete the association of performance with microstructural variance, whether for material design or for material certification, a virtual test must be executed for a statistically significant number of instantiations of the microstructure. Solving typical predictive cases in a reasonable time poses the challenging target of $1 \mathrm{~s}$ as the time, within an order of magnitude, for a single simulation when $10^{4}-10^{6}$ simulations are needed in Monte Carlo strategies. Alternative probabilistic models, in which one tracks the evolution in time of probability density functions for damage rather than tracking the damage itself, offer speed but require new formulations to maintain fidelity. The probabilistic models must include the essential microstructural factors in laws governing the density evolution or else lose fidelity. Ongoing extensions of the virtual-test system for ceramic composites include the incorporation of environmental and other rate effects, especially oxidation and creep at high temperature.

\section{DISCLOSURE STATEMENT}

The authors are not aware of any affiliations, memberships, funding, or financial holdings that might be perceived as affecting the objectivity of this review.

\section{ACKNOWLEDGMENTS}

This work was supported by the US Air Force Office of Scientific Research (AFOSR) (Dr. Ali Sayir) and by NASA (Dr. Anthony Calomino) under the National Hypersonics Science Center for Materials and Structures (AFOSR contract number FA9550-09-1-0477). Drs. Jamie Nasiatka and Alistair MacDowell contributed to the development of the high-temperature $\mu \mathrm{CT}$ apparatus discussed in this review.

\section{LITERATURE CITED}

1. Cox BN, Yang QD. 2006. In quest of virtual tests for structural composites. Science 314:1102-7

2. Ashby MF. 1992. Physical modelling of materials problems. Mater. Sci. Technol. 8:102-11

3. Cox BN, Yang QD. 2009. Virtual tests of laminated composites-adding the sublaminar scale. Presented at ECCOMAS Thematic Conference on the Mechanical Response of Composites, 2nd, April 1-3, London

4. Llorca J, Cox BN, eds. 2010. Virtual fracture testing of composite materials and structures. Proc. World Congr. Comput. Mech., 8th, Venice, June 30-July 4, 2008, in Special Issue, Int. F. Fracture, 158(2)

5. Groeber M, Ghosh S, Uchic MD, Dimiduk DM. 2008. A framework for automated analysis and simulation of 3D polycrystalline microstructures. Part 2: synthetic structure generation. Acta Mater. 56:1274-87

6. Pollock TM, Allison JE, Backman DG, Boyce MC, Gersh M, et al. 2008. Integrated Computational Materials Engineering: A Transformational Discipline for Improved Competitiveness and National Security. Washington, DC: Natl. Res. Counc., Natl. Acad.

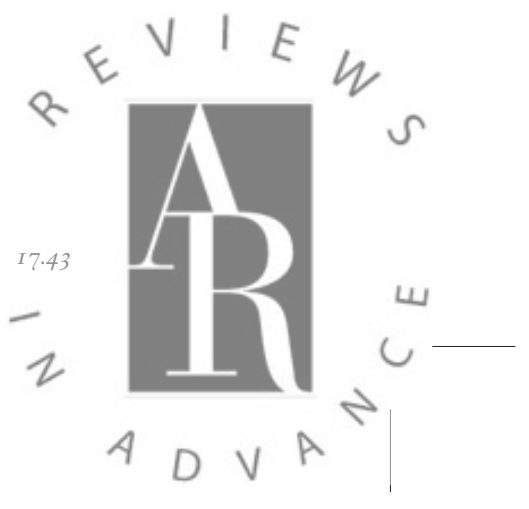


7. Schmitz GJ, Prahl U, eds. 2012. Integrative Computational Materials Engineering: Concepts and Applications of a Modular Simulation Platform. New York: Wiley

8. Badel P, Vidal-Sallé E, Maire E, Boisse P. 2008. Simulation and tomography analysis of textile composite reinforcement deformation at the mesoscopic scale. Compos. Sci. Technol. 68:2433-40

9. Bale H, Blacklock M, Begley MR, Marshall DB, Cox BN, Ritchie RO. 2011. Characterizing threedimensional textile ceramic composites using synchrotron X-ray micro-computed-tomography. 7 . Am. Ceram. Soc. 95:392-402

10. Coindreau O, Vignoles G, Cloetens P. 2003. Direct 3D microscale imaging of carbon-carbon composites with computed holotomography. Nucl. Instrum. Methods B 200:308-14

11. Martin-Herrero J, Germain C. 2007. Microstructure reconstruction of fibrous C-C composites from XMCT. Carbon 45:1242-53

12. Wright P, Fu X, Sinclair I, Spearing SM. 2008. Ultra high resolution computed tomography of damage in notched carbon fiber-epoxy composites. F. Compos. Mater. 42:1993-2002

13. Desplentere F, Lomov SV, Woerdeman DL, Verpoest I, Wevers M, Bogdanovich AE. 2005. Micro-CT characterization of variability in 3D textile architecture. Compos. Sci. Technol. 65:1920-30

14. Cox BN, Spearing SM, Mumm DR. 2008. Practical challenges in formulating virtual tests for structural composites. In Mechanical Response of Composites, ed. PP Camanho, CG Dávila, ST Pinho, JJC Remmers, pp. 57-75. Dordrecht: Springer Sci. Bus. Media

15. Stock SR. 2008. Recent advances in X-ray microtomography applied to materials. Int. Mater. Rev. 53:12981

16. Sakdinawat A, Attwood D. 2010. Nanoscale X-ray imaging. Nat. Photonics 4:840-48

17. de Borst R, Remmers JJC, Needleman A. 2004. Computational aspects of cohesive-zone models. Presented at Eur. Conf. Fract., 15th, Aug. 11-13

18. Ling D, Yang Q, Cox BN. 2009. An augmented finite element method for modeling arbitrary discontinuities in composite materials. Int. F. Fract. 156:53-73

19. Moës N, Dolbow J, Belytschko T. 1999. A finite element method for crack growth without remeshing. Int. F. Numer. Methods Eng. 46:131-50

20. Needleman A. 1990. An Analysis of decohesion along an imperfect interface. Int. F. Fract. 42:21-40

21. Strouboulis T, Copps K, Babuška I. 2001. The generalized finite element method. Comput. Mech. Adv. 190:4081-193

22. Zi G, Belytschko T. 2003. New crack-tip elements for XFEM and applications to cohesive cracks. Int. 7. Numer. Methods Eng. 57:2221-40

23. Fang XJ, Yang Q, Cox BN, Zhou ZQ. 2011. An augmented cohesive zone element for arbitrary crack coalescence and bifurcation in heterogeneous materials. Int. F. Numer. Methods Eng. 88:841-61

24. Fang XJ, Zhou ZQ, Cox BN, Yang Q. 2011. High-fidelity simulations of multiple fracture processes in laminated composites in tension. F. Mech. Phys. Solids 59:1355-73

25. Liu W, Yang QD, Mohammedizadeh S, Su XY, Ling DS. 2013. An accurate and efficient augmented finite element method for arbitrary crack interactions. F. Appl. Mech. 80:041033

26. Pineda EJ, Bednarcyk BA, Waas AM, Arnold SM. 2013. Progressive failure of a unidirectional fiberreinforced composite using the method of cells: discretization objective computational results. Int. $\mathcal{F}$. Solids Struct. 50:1203-16

27. Buehler MJ. 2006. Large-scale hierarchical modeling of nanoscale, natural and biological materials. 7. Comput. Theor. Nanosci. 3:603-23

28. Weinan E, Engquist B, Li X, Ren W, Vanden-Eijnden E. 2007. Heterogeneous multiscale methods: a review. Commun. Comput. Phys. 2:367-450

29. Fish J, ed. 2010. Multiscale Methods: Bridging the Scales in Science and Engineering. Oxford, UK: Oxford Univ. Press

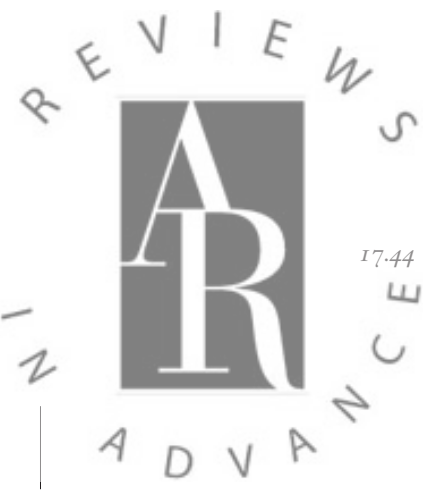

30. González C, Llorca J. 2006. Multiscale modeling of fracture in fiber-reinforced composites. Acta Mater. $54: 4171-81$

31. Moffat AJ, Wright P, Buffiere JY, Sinclair I, Spearing SM. 2008. Micromechanisms of damage in $0^{\circ}$ splits in a [90/0]s composite material using synchrotron radiation computed tomography. Scr. Mater. $59: 1043-46$

Cox et al. 
32. Morscher GN, Yun HM, DiCarlo JA. 2007. In-plane cracking behavior and ultimate strength for 2D woven and braided melt-infiltrated $\mathrm{SiC} / \mathrm{SiC}$ composites tensile loaded in off-axis fiber directions. 7 . Am. Ceram. Soc. 90:3185-93

33. Yun HM, DiCarlo JA. 2004. Through-thickness properties of 2D woven SiC/SiC panels with various microstructures. Ceram. Eng. Sci. Proc. 25:71-78

34. Yun HM, Gyekenyesi JZ, DiCarlo JA. 2002. Effects of 3D-fiber architecture on tensile stress-strain behavior of $\mathrm{SiC} / \mathrm{SiC}$ composites. Ceram. Eng. Sci. Proc. 23:503-10

35. Morscher GN, DiCarlo JA, Kiser JD, Yun HM. 2010. Effects of fiber architecture on matrix cracking for melt-infiltrated SiC/SiC composites. Int. 7. Appl. Ceram. Technol. 7:276-90

36. Cox BN, Dadkhah MS, Inman RV, Morris WL, Zupon J. 1992. Mechanisms of compressive failure in 3D composites. Acta Metall. Mater. 40:3285-98

37. Cox BN, Dadkhah MS, Morris WL. 1996. On the tensile failure of 3D woven composites. Composites A 27:447-58

38. Cox BN, Dadkhah MS, Morris WL, Flintoff JG. 1994. Failure mechanisms of 3D woven composites in tension, compression, and bending. Acta Metall. Mater. 42:3967-84

39. Dadkhah MS, Flintoff JG, Kniveton T, Cox BN. 1995. Simple models for triaxially braided composites. Composites 26:91-102

40. Dadkhah MS, Morris WL, Cox BN. 1995. Compression-compression fatigue in 3D woven composites. Acta Metall. Mater. 43:4235-45

41. Lomov SP, Verpoest I. 2000. Compression of woven reinforcements: a mathematical model. 7. Reinf. Plast. Compos. 19:1329-50

42. Long AC, Souter BJ, Robitaille F, Rudd CD. 2002. Effects of fibre architecture on reinforcement fabric deformation. Plast. Rubber Compos. 31:87-97

43. Miao Y, Zhou E, Wang YQ, Cheeseman BA. 2008. Mechanics of textile mechanics: micro-geometry. Compos. Sci. Technol. 68:1671-78

44. Hay RS, Fair GE, Bouffioux R, Urban E, Morrow J, et al. 2001. Hi-Nicalon ${ }^{\text {TM }}$-SSiC fiber oxidation and scale crystallization kinetics. 7. Am. Ceram. Soc. 94:3983-91

45. Hutchinson JW, Evans AG. 2000. Mechanics of materials: top-down approaches to fracture. Acta Mater. 48:125-35

46. Curtin WA. 1994. In situ fiber strengths in ceramic-matrix composites from fracture mirrors. 7. Am. Ceram. Soc. 77:1075-78

47. Curtin WA. 1998. Stochastic damage evolution and failure in fiber-reinforced composites. Adv. Appl. Mech. 36:163-253

48. Ko FK. 1989. Preform fiber architecture for ceramic-matrix composites. Ceram. Bull. 68:401-14

49. Marshall DB, Cox BN. 2008. Integral textile ceramic structures. Annu. Rev. Mater. Res. 38:425-43

50. Mouritz AP, Bannister MK, Falzon PJ, Leong KH. 1999. Review of applications for advanced threedimensional fibre textile composites. Composites A 30:1445-61

51. Schmidt S, Beyer S, Immich H, Knabe H, Meistring R, Gessler A. 2005. Ceramic matrix composites: a challenge in space-propulsion technology applications. Int. F. Appl. Ceram. Technol. 2:85-96

52. Morscher GN, Pujar VV. 2009. Design guidelines for in-plane mechanical properties of SiC fiberreinforced melt-infiltrated SiC composites. Int. F. Appl. Ceram. Technol. 6:151-63

53. Zhao JC, Westbrook JH. 2003. Ultrahigh-temperature materials for jet engines. MRS Bull. 28:622-30

54. Raj R, Scarmi A, Soraru GD. 2005. The role of carbon in unexpected visco(an)elastic behavior of amorphous silicon oxycarbide above 1273K. F. Non Cryst. Solids 351:2238-43

55. Mahadik Y, Robson Brown KA, Hallett SR. 2010. Characterisation of 3D woven composite internal architecture and effect of compaction. Composites A 41:872-80

56. Lee S-B, Stock SR, Butts MD, Starr TL, Breunig TM, Kinney JH. 1998. Pore geometry in woven fiber structures: 0/90 plain-weave cloth layup preform. F. Mater. Res. 13:1209-17

57. Kinney JH, Breunig TM, Starr TL, Haupt D, Nichols MC, et al. 1993. X-ray tomographic study of chemical vapor infiltration processing of ceramic composites. Science 260:789-92

58. Bale HA, Haboub A, MacDowell AA, Nasiatka J, Parkinson DL, et al. 2013. Real-time quantitative imaging of failure events in ultrahigh-temperature materials under load at unprecedented temperatures above $1700^{\circ}$ C. Nat. Mater. 12:40-46

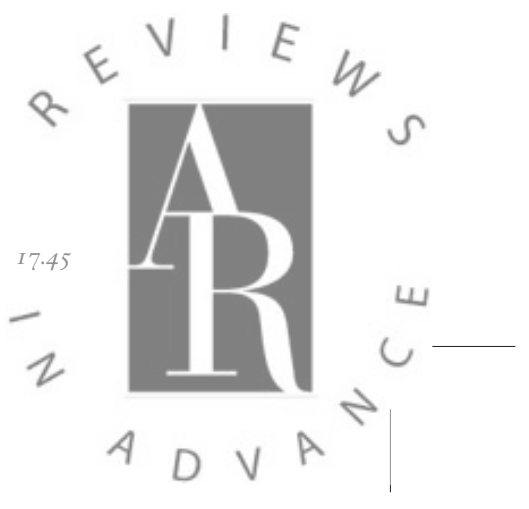


59. Argon AS. 1972. Fracture of composites. In Treatise on Materials Sciences and Technology, Vol. 1, ed. AS Argon, pp. 106-14. New York/London: Academic

60. Budiansky B. 1983. Micromechanics. Compos. Struct. 16:3-12

61. Fleck NA, Budiansky B. 1991. Compressive failure of fibre composites due to microbuckling. In Inelastic Deformation of Composite Materials, ed. GJ Dvorak, pp. 235-74. New York: Springer-Verlag

62. Fleck NA, Shu JY. 1995. Microbuckle initiation in fibre composites: a finite element study. F. Mech. Phys. Solids 43:1887-918

63. Marshall DB, Morris WL, Cox BN, Graves J, Porter JR, et al. 1994. Transverse strengths and failure mechanisms in $\mathrm{Ti}_{3} \mathrm{Al}$ matrix composites. Acta Metall. Mater. 42:2657-73

64. Gereke T, Döbrich O, Hübner M, Cherif C. 2013. Experimental and computational composite textile reinforcement forming: a review. Composites $A$ 46:1-10

65. Boisse P, Gasser A, Hagege B, Billoet J-L. 2005. Analysis of the mechanical behavior of woven fibrous material using virtual tests at the unit cell level. F. Mater. Sci. 40:5955-62

66. Verpoest I, Lomov SV. 2005. Virtual textile composite software WiseTex: integration with micromechanical, permeability, and structural analysis. Compos. Sci. Technol. 65:2563-74

67. Groeber M, Ghosh S, Uchic MD, Dimiduk DM. 2008. A framework for automated analysis and simulation of 3D polycrystalline microstructures. Part 1: statistical characterization. Acta Mater. 56:1257-73

68. Luan J, Liu G, Wang H, Ullah A. 2011. On the sampling of three-dimensional polycrystalline microstructures for distribution determination. 7. Microsc. 244:214-22

69. Rowenhorst D, Gupta A, Feng C, Spanos G. 2006. 3D crystallographic and morphological analysis of coarse martensite: combining EBSD and serial sectioning. Scr. Mater. 55:11-16

70. Uchic MD, Groeber MA, Dimiduk DM, Simmons J. 2006. 3D microstructural characterization of nickel superalloys via serial-sectioning using a dual beam FIB-SEM. Scr. Mater. 55:23-28

71. DeHoff R. 1983. Quantitative serial sectioning analysis: preview. F. Microsc. 131:259-63

72. Khor KH, Buffiere JY, Ludwig W, Toda H, Ubhi HS, et al. 2004. In situ high resolution synchrotron $\mathrm{X}$-ray tomography of fatigue crack closure mechanisms. F. Phys. Condens. Matter 16:S3511-15

73. Drach A, Drach B, Tsukrov I. 2014. Processing of fiber architecture data for finite element modeling of 3D woven composites. Adv. Eng. Softw. In press

74. Blacklock M, Bale H, Begley MR, Cox BN. 2012. Generating virtual textile composite specimens using statistical data from micro-computed tomography: 1D tow representations for the Binary Model. 7. Mech. Phys. Solids 60:451-70

75. Rossol MN, Fast T, Marshall DB, Cox BN, Zok FW. 2014. Characterizing in-plane geometrical variability in textile ceramic composites. Composites B. Submitted

76. Vanaerschot A, Cox BN, Lomov SV, Vandepitte D. 2013. Generation of stochastic macroscopic structures using experimental data of random geometry. Presented at Int. Conf. Textile Compos., 11th (TexComp11), Leuven

77. Cahn JW. 1965. Phase separation by spinodal decomposition in isotropic systems. F. Chem. Phys. 42:9399

78. Gagalowicz A, Ma SD. 1985. Sequential synthesis of natural textures. Comput. Vis. Graph. Image Proc. 30:289-315

79. Julesz B. 1962. Visual pattern discrimination. IRE Trans. Inform. Theory IT-8:84-92

80. Cox BN, Morris WL. 1988. Monte Carlo simulations of the growth of small fatigue cracks. Eng. Fract. Mech. 31:591-610

81. Jiao Y, Stillinger FH, Torquato S. 2009. A superior descriptor of random textures and its predictive capacity. Proc. Natl. Acad. Sci. USA 106:17634-39

82. Yeong CLY, Torquato S. 1998. Reconstruction of random media. Phys. Rev. E 57:495-506

83. Graham-Brady L, Xu XF. 2008. Stochastic morphological modeling of random multiphase materials. 7. Appl. Mech. 75:061001

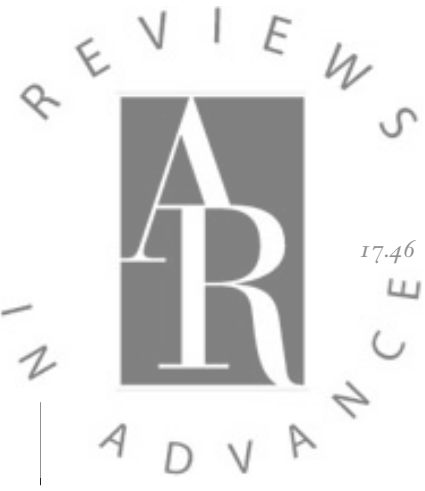

84. Lewis A, Geltmacher A. 2006. Image-based modeling of the response of experimental 3D microstructures to mechanical loading. Scr. Mater. 55:81-85

85. Liu Y, Greene MS, Chen W, Dikin DA, Liu WK. 2013. Computational microstructure characterization and reconstruction for stochastic multiscale material design. Comput. Aided Des. 45:65-76

Cox et al. 
86. Zhang P, Balinta D, Lina J. 2011. Controlled Poisson Voronoi tessellation for virtual grain structure generation: a statistical evaluation. Philos. Mag. 91:4555-73

87. Brahme A, Alvi M, Saylor D, Fridy J, Rollett A. 2006. 3D reconstruction of microstructure in a commercial purity aluminum. Scr. Mater. 55:75-80

88. Hivet G, Boisse P. 2005. Consistent 3D geometrical model of fabric elementary cell. application to a meshing preprocessor for 3D finite element analysis. Finite Elem. Anal. Des. 42:25-49

89. Lomov SV, Verpoest I. 2002. Modelling of the internal structure and deformability of textile reinforcements: WiseTex software. Presented at Eur. Conf. Compos. Mater., 10th (ECCM-10), Brugge, Belg.

90. Pastore CM, Bogdanovich AE, Gowayed YA. 1993. Applications of a meso-volume-based analysis for textile composite structures. Compos. Eng. 3:181-94

91. Sullivan B, Yurus D. 2010. Generation and calibration of 3D woven preform design code for ceramic matrix composite materials. Presented at Annu. Conf. Compos. Mater. Struct., 34th

92. Terpant G, Krishnaswami P, Wang Y. 2002. Computational prediction of yarn structure of 3-D braided composites. In ASTM STP 1416, Composite Materials: Testing, Design, and Acceptance Criteria, ed. A Zereick, AT Nettles, pp. 188-99. West Conshohocken, PA: Am. Soc. Test. Mater. Int.

93. Wang Y, Sun X. 2001. Digital-element simulation of textile processes. Compos. Sci. Technol. 61:311-19

94. Lomov SV, Perie G, Ivanov DS, Verpoest I, Marsal D. 2011. Modeling three-dimensional fabrics and three-dimensional reinforced composites: challenges and solutions. Textile Res. F. 81:28-41

95. Rinaldi R, Blacklock M, Bale H, Begley MR, Cox BN. 2012. Generating virtual textile composite specimens using statistical data from micro-computed tomography: 3D tow representations. F. Mech. Phys. Solids 60:1561-81

96. Cox BN, Carter WC, Fleck NA. 1994. A Binary Model of textile composites. I. Formulation. Acta Metall. Mater. 42:3463-79

97. Yang QD, Cox BN. 2003. Spatially averaged local strains in textile composites via the Binary Model formulation. 7. Eng. Mater. Technol. 125:418-25

98. Yang Q, Cox BN. 2010. Predicting failure in textile composites using the Binary Model with gauge averaging. Eng. Fract. Mech. 77:3174-89

99. Flores S, Evans AG, Zok FW, Genet M, Cox BN, et al. 2010. Treating matrix nonlinearity in the Binary Model formulation for 3D ceramic composite structures. Composites A 41:222-29

100. Xu J, Cox BN, McGlockton MA, Carter WC. 1995. A Binary Model of textile composites. II. Elastic regime. Acta Metall. Mater. 43:3511-24

101. Lomov SV, Ivanov DS, Verpoest I, Zako M, Kurashiki T, et al. 2007. Meso-FE modelling of textile composites: road map, data flow and algorithms. Compos. Sci. Technol. 67:1870-91

102. Miyazaki T, Shimajiri M, Yamada H, Seki Itoh H. 1995. A knitting pattern recognition and stitch symbol generating system for knit designing. Comput. Ind. Eng. 29:669-73

103. Grishanov S, Meshkov V, Omelchenko A. 2009. A topological study of textile structures. Part II. Topological invariants in application to textile structures. Textile Res. F. 79:822-36

104. Grishanov S, Meshkov V, Omelchenko A. 2009. A topological study of textile structures. Part I. An introduction to topological methods. Textile Res. F. 79:702-13

105. Xiao M, Geng Z. 2010. A model of rigid bodies for plain-weave fabrics. Textile Res. 7. 80:1995-2006

106. Rugg KL, Cox BN. 2004. Deformation mechanisms of dry textile preforms under mixed compressive and shear loading. F. Reinf. Plast. Compos. 23:1425-42

107. Blacklock M, Shaw JH, Zok FW, Cox BN. 2013. Calibrated stochastic virtual specimens for analyzing local strain variations in woven ceramic composites. Composites. Submitted

108. Marshall DB, Evans AG. 1985. Failure mechanisms in ceramic-fiber/ceramic-matrix composites. F. Am. Ceram. Soc. 68:225-31

109. Heredia FE, Spearing SM, Evans AG, Mosher P, Curtin WA. 1992. Mechanical properties of continuous fiber reinforced carbon matrix composites and relationships to constituent properties. F. Am. Ceram. Soc. 75:3017-25

110. Wang YL, Anandakumar U, Singh RN. 2000. Effect of fiber bridging stress on the fracture resistance of silicon-carbide-fiber/zircon composites. F. Am. Ceram. Soc. 83:1207-14

111. Cutler WA, Zok FW, Lange FF, Charalambides PG. 1997. Delamination resistance of two hybrid ceramic-composite laminates. 7. Am. Ceram. Soc. 80:3029-37

www.annualreviews.org • Stochastic Virtual Tests

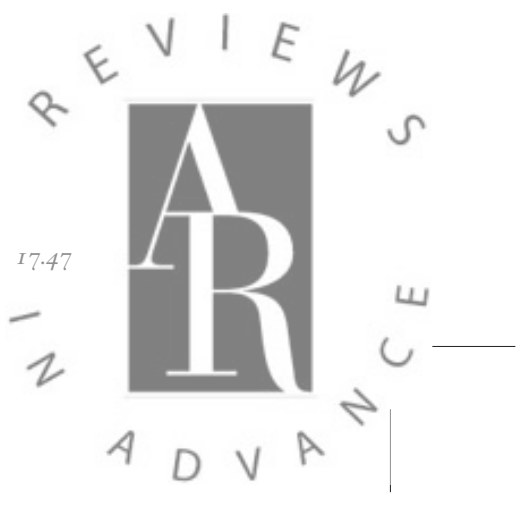


112. McNulty JC, Begley MR, Zok FW. 2001. In-plane fracture resistance of a crossply fibrous monolith. 7. Am. Ceram. Soc. $84: 367-75$

113. Spearing SM, Zok FW, Evans AG. 1994. Stress corrosion cracking in a unidirectional ceramic-matrix composite. F. Am. Ceram. Soc. 77:562-70

114. Morris WL, Cox BN, Marshall DB, Inman RV, James MR. 1994. Fatigue mechanisms in graphite/SiC composites at room and high temperatures. 7. Am. Ceram. Soc. 77:792-800

115. Kodama H, Sakamoto H, Miyoshi T. 1989. Silicon carbide monofilament-reinforced silicon nitride or silicon carbide matrix composites. F. Am. Ceram. Soc. 72:551-58

116. Barsoum MW, Kangutkar P, Wang ASD. 1992. Matrix crack initiation in ceramic matrix composites. Part I. Experiments and test results. Compos. Sci. Technol. 44:257-69

117. Kaute DAW, Shercliff HR, Ashby MF. 1993. Delamination, fibre bridging and toughness of ceramic matrix composites. Acta Metall. Mater. 41:1959-70

118. Liu Y, Tanaka Y. 2003. In situ characterization of tensile damage behavior of a plain-woven fiberreinforced polymer-derived ceramic composite. Mater. Lett. 57:1571-78

119. Shercliff HR, Vekinis G, Beaumont PWR. 1994. Direct observation of the fracture of CAS-glass/SiC composites. F. Mater. Sci. 29:3643-52

120. Rugg KL, Dadkhah MS, Berbon MZ, Marshall DB. 1999. Strain measurement in woven ceramic matrix composites using laser speckle interferometry. Ceram. Trans. 103:549-57

121. Berbon MZ, Rugg KL, Dadkhah MS, Marshall DB. 2002. Effect of weave architecture on tensile properties and local strain heterogeneity in thin-sheet C-SiC composites. F. Am. Ceram. Soc. 85:2039-48

122. Yang QD, Rugg KL, Cox BN, Marshall DB. 2005. Evaluation of macroscopic and local strains in a 3D woven C/SiC composite. 7. Am. Ceram. Soc. 88:719-25

123. Morscher GN. 1999. Modal acoustic emission of damage accumulation in a woven $\mathrm{SiC} / \mathrm{SiC}$ composite. Compos. Sci. Technol. 59:687-97

124. Morscher GN. 2004. Stress-dependent matrix cracking in 2D woven SiC-fiber reinforced melt-infiltrated SiC matrix composites. Compos. Sci. Technol. 64:1311-19

125. Morscher GN, Ojard G, Miller R, Gowayed Y, Santhosh U, et al. 2008. Tensile creep and fatigue of Sylramic-iBN melt-infiltrated SiC matrix composites: retained properties, damage development, and failure mechanisms. Compos. Sci. Technol. 68:3305-13

126. Smith CE, Morscher GN, Xia Z. 2011. Electrical resistance as a nondestructive evaluation technique for $\mathrm{SiC} / \mathrm{SiC}$ ceramic matrix composites under creep-rupture loading. Int. F. Appl. Ceram. Technol. 8:298-307

127. Smith CE, Morscher GN, Xia ZH. 2008. Monitoring damage accumulation in ceramic matrix composites using electrical resistivity. Scr. Mater. 59:463-66

128. Kanka B, Schneider H. 2000. Aluminosilicate fiber/mullite matrix composites with favorable hightemperature properties. F. Eur. Ceram. Soc. 20:619-23

129. Terzi S, Salvoa L, Suérya M, Limodinb N, Adrienb J, et al. 2009. In situ X-ray tomography observation of inhomogeneous deformation in semi-solid aluminium alloys. Scr. Mater. 61:449-52

130. Kinney JH, Nichols MC. 1992. X-ray tomographic microscopy (XTM) using synchrotron radiation. Annu. Rev. Mater. Sci. 22:121-52

131. Château C, Gélébart L, Bornert M, Crépin J, Boller E, et al. 2011. In situ X-ray microtomography characterization of damage in $\mathrm{SiC}_{\mathrm{f}} / \mathrm{SiC}$ minicomposites. Compos. Sci. Technol. 71:916-24

132. Château C, Gélébart L, Bornert M, Crépin J, Caldemaison D, et al. 2010. Experimental minicomposites. Presented at ICEM Int. Conf. Exp. Mech., 14th (ICEM 14), Poitiers, Fr.

133. Chu T, Ranson W, Sutton M. 1985. Applications of digital-image-correlation techniques to experimental mechanics. Exp. Mech. 25:232-44

134. Hild F, Roux S. 2006. Digital image correlation: from displacement measurement to identification of elastic properties—a review. Strain 42:69-80

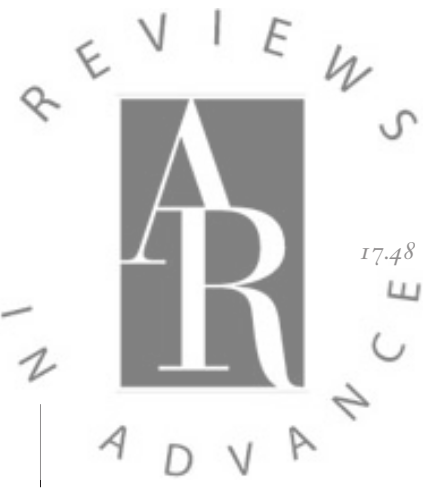

135. McCormick N, Lord J. 2010. Digital image correlation. Mater. Today 13:52-54

136. Vendroux G, Knauss W. 1998. Submicron deformation field measurements. Part 2. Improved digital image correlation. Exp. Mech. 38:86-92

137. James MR, Morris WL, Cox BN. 1990. A high accuracy automated strain field mapper. Exp. Mech. 30:60-67

Cox et al.

Changes may still occur before final publication online and in print 
138. Novak MD, Zok FW. 2011. High-temperature materials testing with full-field strain measurement: experimental design and practice. Rev. Sci. Instrum. 82:115101

139. Shaw JH, Rajan VP, Blacklock M, Zok FW. 2014. Towards a virtual test for C/SiC textile composites: calibration of thermoelastic tow properties. 7. Am. Ceram. Soc. In press

140. Yang J-M, Ma C-L, Chou T-W. 1986. Fiber inclination model of three-dimensional textile structural composites. 7. Compos. Mater. 20:472-84

141. Cox BN, Dadkhah MS. 1995. The macroscopic elasticity of 3D woven composites. F. Compos. Mater. 29:785-819

142. Bogdanovich A, Pastore CM. 1996. Mechanics of Textile and Laminated Composites: With Applications to Structural Analysis. Berlin: Springer

143. Morscher GN, Yun H-M, DiCarlo JA. 2005. Matrix cracking in 3D orthogonal melt-infiltrated SiC/SiC composites with various Z-fiber types. 7. Am. Ceram. Soc. 88:146-53

144. Ryou H, Chung K, Yu W-R. 2007. Constitutive modeling of woven composites considering asymmetric/anisotropic, rate dependent, and nonlinear behavior. Composites A 38:2500-10

145. Yanjun C, Guiqiong J, Bo W, Wei L. 2006. Elastic behavior analysis of 3D angle-interlock woven ceramic composites. Acta Mech. Solida Sin. 19:152-59

146. Takano N, Zako M, Kubo F, Kimura K. 2003. Microstructure-based stress analysis and evaluation for porous ceramics by homogenization method with digital image-based modeling. Int. 7. Solids Struct. 40:1225-42

147. Fish J, Yu Q. 2001. Two-scale damage modeling of brittle composites. Compos. Sci. Technol. 61:2215-22

148. Pineau P, Couegnat G, Lamon J. 2011. Virtual testing applied to transverse multiple cracking of tows in woven ceramic composites. Mech. Res. Commun. 38(8):579-85

149. Lamon J. 2001. A micromechanics-based approach to the mechanical behavior of brittle-matrix composites. Compos. Sci. Technol. 61:2259-72

150. Chang Y-J, Jiao G-Q, Wang B, Guan G-Y, Lu Z-X. 2007. Mechanical properties and damage process of a three-dimensional woven ceramic composite under in-plane shear loading. F. Inorg. Mater. 1:023

151. Genin GM, Hutchinson JW. 1997. Composite laminates in plane stress: constitutive modeling and stress redistribution due to matrix cracking. 7. Am. Ceram. Soc. 80:1245-55

152. Rajan VP, Zok FW. 2014. Remediation of a constitutive model for ceramic composite laminates. Composites. In press

153. Clarke JD, McGregor IJ. 1993. Ultimate tensile criterion over a zone: a new failure criterion for adhesive joints. 7. Adhes. 42:227-45

154. Feih S, Shercliffe HR. 2004. Adhesive and composite failure prediction of single-L joint structures under tensile loading. Int. 7. Adhes. Adhes. 25:47-59

155. Rossmanith HP. 1995. An introduction to K. Wieghardt's historical paper "On splitting and fracture of elastic bodies." Fatigue Fract. Eng. Mater. Struct. 12:1367-69

156. Sheppard A, Kelly D, Tong L. 1998. A damage zone model for the failure analysis of adhesively bonded joints. Int. F. Adhes. Adhes. 18:385-400

157. Lawn BR. 1993. Fracture of brittle solids. In Cambridge Solid State Science Series, ed. EA Davis, IM Ward, p. 378. Cambridge, UK: Cambridge Univ. Press. 2nd ed.

158. Bao G, Suo Z. 1992. Remarks on crack-bridging concepts. Appl. Mech. Rev. 24:355-66

159. Carpinteri A, Massabò R. 1996. Bridged versus cohesive crack in the flexural behavior of brittle matrix composites. Int. F. Fract. 81:125-45

160. Cox BN, Marshall DB. 1994. Concepts for bridged cracks in fracture and fatigue. Acta Metall. Mater. 42:341-63

161. Pineau P, Couegnat G, Lamon J. 2011. Virtual testing applied to transverse multiple cracking of tows in woven ceramic composites. Mech. Res. Commun. 38:7

162. Yang QD, Thouless MD. 2001. Mixed mode fracture of plastically-deforming adhesive joints. Int. F. Fract. 110:175-87

163. Marshall DB, Cox BN, Evans AG. 1985. The mechanics of matrix cracking in brittle-matrix fiber composites. Acta Metall. 33:2013-21

164. McCartney LN. 1987. Mechanics of matrix cracking in brittle-matrix fibre-reinforced composites. Proc. R. Soc. $A$ 409:329-50

www.annualreviews.org • Stochastic Virtual Tests

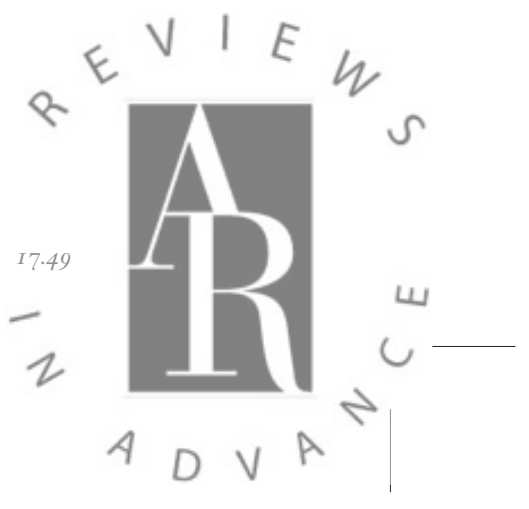


165. Aveston J, Cooper GA, Kelly A. 1971. Single and multiple fracture. Proc. Conf. Prop. Fibre Compos., London, pp. 15-26. Guildford, UK: IPC Sci. Technol.

166. Cox BN. 1990. Interfacial sliding near a free surface in a fibrous or layered composite during thermal cycling. Acta Metall. Mater. 38:2411-24

167. Babuška I, Melenk JM. 1997. The partition of unity method. Int. F. Numer. Methods Eng. 40:727-58

168. Melenk JM, Babuška I. 1996. The partition of unity finite element method: basic theory and applications. Comput. Methods Appl. Mech. Eng. 139:289-314

169. Duarte CA, Babuška I, Oden JT. 2000. Generalized finite element methods for three-dimensional structural mechanics problems. Comput. Struct. 77:215-32

170. Strouboulis T, Babuška I, Copps K. 2000. The design and analysis of the Generalized Finite Element Method. Comput. Methods Appl. Mech. Eng. 181:43-69

171. Strouboulis T, Copps K, Babuška I. 2001. Computational mechanics advances. The generalized finite element method. Comput. Methods Appl. Mech. Eng. 190:4081-193

172. Strouboulis T, Copps K, Babuška I. 2001. The generalized finite element method. Comput. Methods Appl. Mech. Eng. 190:4081-193

173. Daux C, Moës N, Dolbow J, Sukumar N, Belytschko T. 2000. Arbitrary branched and intersecting cracks with the extended finite element method. Int. F. Numer. Methods Eng. 48:1741-60

174. Stolarska M, Chopp DL, Moës N, Belytschko T. 2001. Modelling crack growth by level sets in the extended finite element method. Int. F. Numer. Methods Eng. 51:943-60

175. Moës N, Belytschko T. 2002. Extended finite element method for cohesive crack growth. Eng. Fract. Mech. 69:813-33

176. Ventura G, Xu JX, Belytschko T. 2002. A vector level set method and new discontinuity approximations for crack growth by EFG. Int. 7. Numer. Methods Eng. 54:923-44

177. Legay A, Chessa J, Belytschko T. 2006. An Eulerian-Lagrangian method for fluid-structure interaction based on level sets. Comput. Methods Appl. Mech. Eng. 195:2070-87

178. Xiao QZ, Karihaloo BL. 2007. Implementation of hybrid crack element on a general finite element mesh and in combination with XFEM. Comput. Methods Appl. Mech. Eng. 196:1864-73

179. Belytschko T, Gracia R, Ventura G. 2009. A review of extended/generalized finite element methods for material modeling. Int. 7. Numer. Methods Eng. 86:637-66

180. Hansbo A, Hansbo P. 2002. An unfitted finite element method, based on Nitsche's method, for elliptic interface problems. Comput. Methods Appl. Mech. Eng. 191:5537-52

181. Hansbo A, Hansbo P. 2004. A finite element method for the simulation of strong and weak discontinuities in solid mechanics. Comput. Methods Appl. Mech. Eng. 193:3523-40

182. Song JH, Areias PMA, Belytschko T. 2006. A method for dynamic crack and shear band propagation with phantom nodes. Inter. F. Numer. Meth. Eng. 67:868-93

183. Van de Meer FP, Sluys LJ. 2009. Continuum models for the analysis of progressive failure in composite laminates. 7. Compos. Mater. 43:2131-56

184. Van de Meer FP, Sluys LJ. 2009. A phantom node formulation with mixed mode cohesive law for splitting in laminates. Int. 7. Fract. 158:107-24

185. Van de Meer FP, Oliver C, Sluys LJ. 2010. Computational analysis of progressive failure in a notched laminate including shear nonlinearity and fiber failure. Compos. Sci. Technol. 70:692-700

186. Ling DS, Yang QD, Cox BN. 2009. An augmented finite element method for modeling arbitrary discontinuities in composite materials. Int. F. Fract. 156:53-73

187. Ling DS, Fang XJ, Cox BN, Yang QD. 2011. Nonlinear fracture analysis of delamination crack jumps in laminated composites. F. Aerosp. Eng. 24:181-88

188. Fang XJ, Zhou ZQ, Cox BN, Yang QD. 2011. High-fidelity simulations of multiple fracture processes in a laminated composite in tension. F. Mech. Phys. Solids 59:1355-73

189. Xu XP, Needleman A. 1994. Numerical simulations of fast crack growth in brittle solids. F. Mech. Phys. Solids 42:1397-434

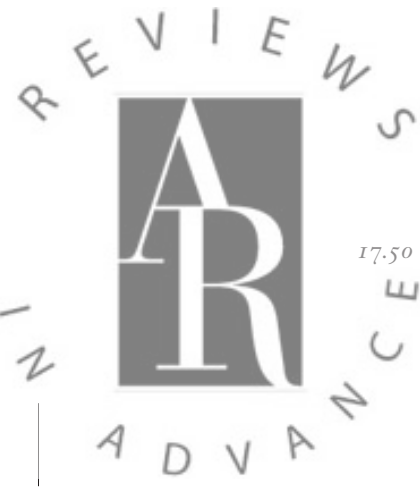

190. Turon A, Camanho PP, Costa J, Davila CG. 2006. A damage model for the simulation of delamination in advanced composites under variable-mode loading. Mech. Mater. 38:1072-89

191. Yang QD, Cox BN. 2005. Cohesive models for damage evolution in laminated composites. Int. F. Fract. $133: 107-37$

Cox et al. 
192. Van de Meer FP. 2012. Mesolevel modeling of failure in composite laminates: constitutive, kinematic and algorithmic aspects. Arch. Comput. Method Eng. 19:381-425

193. Iarve EV, Mollenhauer D, Kim R. 2005. Theoretical and experimental investigation of stress redistribution in open-hole composite laminates due to damage accumulation. Composites A 36:163-71

194. Iarve EV. 2003. Mesh independent modelling of cracks by using higher order shape functions. Int. $\mathcal{F}$. Numer. Methods Eng. 56:869-82

195. Iarve EV, Gurvich MR, Mollenhauer D, Rose CA, Davila CG. 2011. Mesh independent matrix cracking and delamination modelling in laminated composites. Int. F. Numer. Methods Eng. 88:749-73

196. Liu W, Yang QD, Mohammadizadeh S, Su XY. 2014. An efficient augmented finite element method (A-FEM) for arbitrary cracking and crack interaction in solids. Int. 7. Numer. Methods Eng. In press

197. Ho S, Suo Z. 1993. Tunneling cracks in constrained layers. F. Appl. Mech. 60:890-94

198. Ortiz K, Kiremidjian AS. 1988. Stochastic modelling of fatigue crack growth. Eng. Fract. Mech. 29:317-34

199. Ellyin F. 1997. Fatigue Damage, Crack Growth and Life Prediction. Berlin: Springer

200. Bogdanoff JL. 1978. A new cumulative damage model. Part I. 7. Appl. Mech. 45:246-50

201. Bogdanoff JL, Kozin F. 1985. Probabilistic Models of Cumulative Damage. New York: Wiley

202. Sobczyk K. 1986. Modelling of random fatigue crack growth. Eng. Fract. Mech. 24:609-23

203. Ghonem H, Dore S. 1985. Probabilistic description of fatigue crack growth in polycrystalline solids. Eng. Fract. Mech. 21:1151-68

204. Lin Y, Yang J. 1985. A stochastic theory of fatigue crack propagation. AIAA f. 23:117-24

205. Ritchie RO, Lankford J, eds. 1986. Small Fatigue Cracks. Warrendale, PA: Metall. Soc.

206. Lankford J. 1985. The influence of microstructure on the growth of small fatigue cracks. Fatigue Fract. Eng. Mater. Struct. 8:161-75

207. Metropolis N, Ulam S. 1949. The Monte Carlo method. F. Am. Stat. Assoc. 44:335-41

208. Cox BN. 1989. Inductions from Monte Carlo simulations of small fatigue cracks. Eng. Fract. Mech. 33:655-70

209. Morris WL, James MR, Buck O. 1981. Growth rate models for short surface cracks in Al 2219-T851. Metall. Trans. A 12:57-64

210. Fokker AD. 1914. Die mittlere Energie rotierender elektrischer Dipole im Strahlungsfeld. Ann. Phys. 348:810-20

211. Gardiner C. 2009. Stochastic Methods. Berlin: Springer

212. Papoulis A. 1984. Probability, Random Variables, and Stochastic Processes. New York: McGraw-Hill

213. Bogdanoff JL, Kozin F. 1982. On nonstationary cumulative damage models. F. Appl. Mech. 49:37-42

214. Howard RA. 1970. Dynamic Probabilistic Systems. New York: Wiley

215. Limnios G, Oprisan G. 2001. Semi-Markov Processes and Reliability. Boston: Birkhauser

216. Cox BN, Morris WL. 1987. A probabilistic model of short fatigue crack growth. Fatigue Fract. Eng. Mater. Struct. 10:419-28

217. Cox BN, Morris WL. 1987. Model-based statistical analysis of short fatigue crack growth in Ti 6Al4Sn-2Zr-6Mo. Fatigue Fract. Eng. Mater. Struct. 10:429-46

218. Cox BN, Pardee WJ, Morris WL. 1987. A statistical model of intermittent short fatigue crack growth. Fatigue Fract. Eng. Mater. Struct. 9:435-55

219. Pardee WJ, Morris WL, Cox BN, Hughes BD. 1982. Statistical mechanics of early growth of fatigue cracks. Int. Symp. on Defects, Fracture and Fatigue, 2nd, Mont Gabriel, Can., pp. 99-111

220. Fast T, Scott AE, Bale HA, Cox BN. 2014. Topological and Euclidean metrics for stochastic fiber bundles. Composites A. Submitted

221. Voronoi G. 1907. Nouvelles applications des paramètres continus à la théorie des formes quadratiques. 7. Reine Angew. Math. 133:97-178

222. Alfaro MC, Suiker A, De Borst R. 2010. Transverse failure behavior of fiber-epoxy systems. F. Compos. Mater. 44:1493-516

223. Brockenbrough JR, Suresh S, Wienecke HA. 1991. Deformation of fiber-reinforced metal-matrix composites: geometrical effects of fiber shape and distribution. Acta Metall. Mater. 39:735-52

224. Lorenz EN. 1963. Deterministic nonperiodic flow. F. Atmos. Sci. 20:130-41

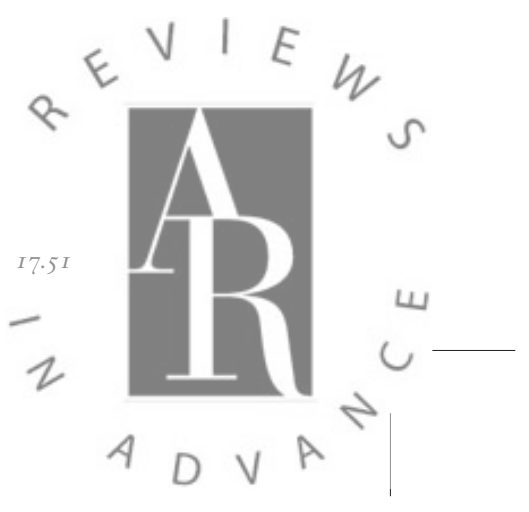

